\title{
The algebro-geometric Toda hierarchy initial value problem for complex-valued initial data
}

Fritz Gesztesy, Helge Holden and Gerald Teschl

\begin{abstract}
We discuss the algebro-geometric initial value problem for the Toda hierarchy with complex-valued initial data and prove unique solvability globally in time for a set of initial (Dirichlet divisor) data of full measure. To this effect we develop a new algorithm for constructing stationary complex-valued algebro-geometric solutions of the Toda hierarchy, which is of independent interest as it solves the inverse algebro-geometric spectral problem for generally non-self-adjoint Jacobi operators, starting from a suitably chosen set of initial divisors of full measure. Combined with an appropriate first-order system of differential equations with respect to time (a substitute for the wellknown Dubrovin equations), this yields the construction of global algebro-geometric solutions of the time-dependent Toda hierarchy.

The inherent non-self-adjointness of the underlying Lax (i.e., Jacobi) operator associated with complex-valued coefficients for the Toda hierarchy poses a variety of difficulties that, to the best of our knowledge, are successfully overcome here for the first time. Our approach is not confined to the Toda hierarchy but applies generally to $1+1$-dimensional completely integrable discrete soliton equations.
\end{abstract}

\section{Introduction}

The principal aim of this paper is an explicit construction of unique global solutions of the algebro-geometric initial value problem for the Toda hierarchy with complex-valued initial data. More precisely, we intend to describe

2000 Mathematics Subject Classification: Primary 37K10, 37K20, 47B36; Secondary 35Q58, 37K60.

Keywords: Toda hierarchy, complex-valued solutions, initial value problem. 
a solution of the following problem: Given $p \in \mathbb{N}_{0}$, assume $a^{(0)}, b^{(0)}$ to be complex-valued solutions of the $p$ th stationary Toda system $\mathrm{s}^{-\mathrm{Tl}_{p}}(a, b)=0$ associated with a prescribed nonsingular hyperelliptic curve $\mathcal{K}_{p}$ of genus $p$ and let $r \in \mathbb{N}_{0}$; we want to construct unique global solutions $a=a\left(t_{r}\right)$, $b=b\left(t_{r}\right)$ of the $r$ th $\mathrm{Tl}$ flow $\mathrm{Tl}_{r}(a, b)=0$ with $a\left(t_{0, r}\right)=a^{(0)}, b\left(t_{0, r}\right)=b^{(0)}$ for some $t_{0, r} \in \mathbb{R}$. Thus, we seek a unique global solution of the initial value problem

$$
\begin{aligned}
& \mathrm{Tl}_{r}(a, b)=0 \\
& \left.(a, b)\right|_{t_{r}=t_{0, r}}=\left(a^{(0)}, b^{(0)}\right) \\
& \mathrm{s}-\mathrm{Tl}_{p}\left(a^{(0)}, b^{(0)}\right)=0
\end{aligned}
$$

for some $t_{0, r} \in \mathbb{R}, p, r \in \mathbb{N}_{0}$, where $a=a\left(n, t_{r}\right), b=b\left(n, t_{r}\right)$ satisfy

$$
\begin{aligned}
& a: \mathbb{Z} \times \mathbb{R} \rightarrow \mathbb{C} \backslash\{0\}, \quad b: \mathbb{Z} \times \mathbb{R} \rightarrow \mathbb{C}, \\
& a(\cdot, t), b(\cdot, t) \in \mathbb{C}^{\mathbb{Z}}, t \in \mathbb{R}, \quad a(n, \cdot), b(n, \cdot) \in C^{1}(\mathbb{R}), n \in \mathbb{Z} .
\end{aligned}
$$

In the special case of a self-adjoint Lax (i.e., Jacobi) operator $L$, where $a$ and $b$ are real-valued and bounded, the actual solution of this algebrogeometric initial value problem consists of the following two-step procedure discussed in detail in [6] (see also [14, Sect. 1.3], [32, Sect. 8.3]): ${ }^{1}$

(i) An algorithm that constructs finite nonspecial divisors $\mathcal{D}_{\hat{\mu}(n)} \in \operatorname{Sym}^{p}\left(\mathcal{K}_{p}\right)$ in real position for all $n \in \mathbb{Z}$ starting from an initial Dirichlet divisor $\mathcal{D}_{\hat{\mu}\left(n_{0}\right)} \in$ $\operatorname{Sym}^{p}\left(\mathcal{K}_{p}\right)$ in an appropriate real position (i.e., with Dirichlet eigenvalues in appropriate spectral gaps of $L)$. "Trace formulas" of the type (3.25) and (3.26) then construct the stationary real-valued solutions $a^{(0)}, b^{(0)}$ of $\mathrm{s}-\mathrm{Tl}_{p}(a, b)=0$.

(ii) The first-order Dubrovin-type system of differential equations (5.42), augmented by the initial divisor $\mathcal{D}_{\hat{\underline{\mu}}\left(n_{0}, t_{0, r}\right)}=\mathcal{D}_{\hat{\hat{\mu}}\left(n_{0}\right)}$ together with the anal-

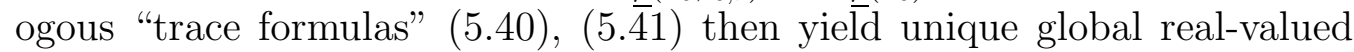
solutions $a=a\left(t_{r}\right), b=b\left(t_{r}\right)$ of the $r$ th $\mathrm{Tl}$ flow $\mathrm{Tl}_{r}(a, b)=0$ satisfying $a\left(t_{0, r}\right)=a^{(0)}, b\left(t_{0, r}\right)=b^{(0)}$.

This approach works perfectly in the special self-adjoint case where the Dirichlet divisors $\hat{\mu}\left(n, t_{r}\right)=\left(\hat{\mu}_{1}\left(n, t_{r}\right), \ldots, \hat{\mu}_{p}\left(n, t_{r}\right)\right) \in \operatorname{Sym}^{p}\left(\mathcal{K}_{p}\right),\left(n, t_{r}\right) \in$ $\mathbb{Z} \times \mathbb{R}$, yield Dirichlet eigenvalues $\mu_{1}\left(n, t_{r}\right), \ldots, \mu_{p}\left(n, t_{r}\right)$ of the Lax operator $L$ situated in $p$ different spectral gaps of $L$ on the real axis. In particular, for fixed $\left(n, t_{r}\right) \in \mathbb{Z} \times \mathbb{R}$, the Dirichlet eigenvalues $\mu_{j}\left(n, t_{r}\right), j=1, \ldots, p$, are pairwise distinct and formulas (5.41) for $a$ and (5.42) for $\left(d / d t_{r}\right) \mu_{j}\left(n, t_{r}\right)$, $j=1, \ldots, p$, are well-defined.

\footnotetext{
${ }^{1}$ We freely use the notation of divisors of degree $p$ as introduced in Appendix A.
} 
This situation drastically changes if complex-valued initial data $a^{(0)}, b^{(0)}$ or $\mathcal{D}_{\hat{\mu}\left(n_{0}, t_{0, r}\right)}$ are permitted. In this case the Dirichlet eigenvalues $\mu_{j}\left(n, t_{r}\right)$, $j=\overline{1}, \ldots, p$, are no longer confined to well separated spectral gaps of $L$ on the real axis and, in particular, they are in general no longer pairwise distinct and "collisions" between them can occur at certain values of $\left(n, t_{r}\right) \in \mathbb{Z} \times \mathbb{R}$. Thus, the stationary algorithm in step $(i)$ as well as the Dubrovin-type firstorder system of differential equations (5.42) in step (ii) above, breaks down at such values of $\left(n, t_{r}\right)$. A priori, one has no control over such collisions, especially, it is not possible to identify initial conditions $\mathcal{D}_{\hat{\mu}\left(n_{0}, t_{0, r}\right)}$ at some $\left(n_{0}, t_{0, r}\right) \in \mathbb{Z} \times \mathbb{R}$ which avoid collisions for all $\left(n, t_{r}\right) \in \mathbb{Z} \times \overline{\mathbb{R}}$. We solve this problem head on by explicitly permitting collisions in the stationary as well as time-dependent context from the outset. In the stationary context, we properly modify the algorithm described above in step $(i)$ in the self-adjoint case by alluding to a more general interpolation formalism (cf. Appendix B) for polynomials, going beyond the usual Lagrange interpolation formulas. In the time-dependent context we replace the first-order system of Dubrovintype equations (5.42), augmented with the initial divisor $\mathcal{D}_{\hat{\mu}\left(n_{0}, t_{0, r}\right)}$, by a different first-order system of differential equations (6.27) with initial conditions (6.28) which focuses on symmetric functions of $\mu_{1}\left(n, t_{r}\right), \ldots, \mu_{p}\left(n, t_{r}\right)$ rather than individual Dirichlet eigenvalues $\mu_{j}\left(n, t_{r}\right), j=1, \ldots, p$. In this manner it will be shown that collisions of Dirichlet eigenvalues no longer pose a problem.

In addition, there is a second nontrivial complication in the non-selfadjoint case: Since the Dirichlet eigenvalues $\mu_{j}\left(n, t_{r}\right), j=1, \ldots, p$, are no longer confined to spectral gaps of $L$ on the real axis as $\left(n, t_{r}\right)$ vary in $\mathbb{Z} \times \mathbb{R}$, it can no longer be guaranteed that $\mu_{j}\left(n, t_{r}\right), j=1, \ldots, p$, stay finite for all $\left(n, t_{r}\right) \in \mathbb{Z} \times \mathbb{R}$. As discussed in Section 4 in the stationary case, this phenomenon is related to certain deformations of the algebraic curve $\mathcal{K}_{p}$ under which for some $n_{0} \in \mathbb{Z}, a\left(n_{0}\right) \rightarrow 0$ and $\mu_{j}\left(n_{0}+1\right) \rightarrow \infty$ for some $j \in\{1, \ldots, p\}$. We solve this particular problem in the stationary as well as time-dependent case by properly restricting the initial Dirichlet divisors $\mathcal{D}_{\underline{\hat{\mu}}\left(n_{0}, t_{0, r}\right)} \in \operatorname{Sym}^{p}\left(\mathcal{K}_{p}\right)$ to a dense set of full measure.

Summing up, we offer a new algorithm to solve the inverse algebrogeometric spectral problem for generally non-self-adjoint Jacobi operators, starting from a properly chosen dense set of initial divisors of full measure. Combined with an appropriate first-order system of differential equations with respect to time (a substitute for the well-known Dubrovin equations), this yields the construction of global algebro-geometric solutions of the timedependent Toda hierarchy.

We emphasize that the approach described in this paper is not limited to the Toda hierarchy but applies universally to constructing algebro-geometric solutions of $1+1$-dimensional integrable discrete soliton equations. In par 
ticular, it applies to differential-difference (i.e., lattice) systems and we are now in the process of applying it to the Ablowitz-Ladik hierarchy. Moreover, the principal idea of replacing Dubrovin-type equations by a first-order system of the type (6.27) is also relevant in the context of general non-selfadjoint Lax operators for continuous models in 1+1-dimensions. (In particular, the models studied in detail in [13] can be revisited from this point of view.) We also note that while the periodic case with complex-valued $a, b$ is of course included in our analysis, we throughout consider the more general algebro-geometric case (in which $a, b$ need not even be quasi-periodic).

Finally we briefly describe the content of each section. Section 2 presents a quick summary of the basics of the Toda hierarchy, its recursive construction, Lax pairs, and zero-curvature equations. The stationary algebrogeometric Toda hierarchy solutions, the underlying hyperelliptic curve, trace formulas, etc., are the subject of Section 3. A new algorithm solving the algebro-geometric inverse spectral problem for generally non-self-adjoint Jacobi operators is presented in Section 4. In Section 5 we briefly summarize the properties of algebro-geometric time-dependent solutions of the Toda hierarchy and formulate the algebro-geometric initial value problem. Uniqueness and existence of global solutions of the algebro-geometric initial value problem as well as their explicit construction are then presented in our final and principal Section 6. Appendix A reviews the basics of hyperelliptic Riemann surfaces of the Toda-type and sets the stage of much of the notation used in this paper. Various interpolation formulas of fundamental importance to our stationary inverse spectral algorithm developed in Section 4 are summarized in Appendix B. Finally, Appendix C summarizes asymptotic spectral parameter expansions of various quantities fundamental to the polynomial recursion formalism presented in Section 2. These appendices support our intention to make this paper reasonably self-contained.

\section{The Toda hierarchy in a nutshell}

In this section we briefly review the recursive construction of the Toda hierarchy and associated Lax pairs and zero-curvature equations following [6], [14, Sect. 1.2], and [32, Ch. 12].

Throughout this section we make the following assumption:

Hypothesis 2.1. Suppose

$$
a, b \in \mathbb{C}^{\mathbb{Z}} \text { and } a(n) \neq 0 \text { for all } n \in \mathbb{Z} \text {. }
$$

Here $\mathbb{C}^{J}$ denotes the set of complex-valued sequences indexed by $J \subseteq \mathbb{Z}$. 
We consider the second-order Jacobi difference expression

$$
L=a S^{+}+a^{-} S^{-}+b,
$$

where $S^{ \pm}$denote the shift operators

$$
\left(S^{ \pm} f\right)(n)=f^{ \pm}(n)=f(n \pm 1), \quad n \in \mathbb{Z}, f \in \mathbb{C}^{\mathbb{Z}} .
$$

To construct the stationary Toda hierarchy we need a second difference expression of order $2 p+2, p \in \mathbb{N}_{0}$, defined recursively in the following. We take the quickest route to the construction of $P_{2 p+2}$, and hence to the Toda hierarchy, by starting from the recursion relations (2.4)-(2.6) below.

Define $\left\{f_{\ell}\right\}_{\ell \in \mathbb{N}_{0}}$ and $\left\{g_{\ell}\right\}_{\ell \in \mathbb{N}_{0}}$ recursively by

$$
\begin{aligned}
& f_{0}=1, \quad g_{0}=-c_{1}, \\
& 2 f_{\ell+1}+g_{\ell}+g_{\ell}^{-}-2 b f_{\ell}=0, \quad \ell \in \mathbb{N}_{0}, \\
& g_{\ell+1}-g_{\ell+1}^{-}+2\left(a^{2} f_{\ell}^{+}-\left(a^{-}\right)^{2} f_{\ell}^{-}\right)-b\left(g_{\ell}-g_{\ell}^{-}\right)=0, \quad \ell \in \mathbb{N}_{0} .
\end{aligned}
$$

Explicitly, one finds

$$
\begin{aligned}
& f_{0}=1, \\
& f_{1}=b+c_{1}, \\
& f_{2}=a^{2}+\left(a^{-}\right)^{2}+b^{2}+c_{1} b+c_{2}, \text { etc. } \\
& g_{0}=-c_{1}, \\
& g_{1}=-2 a^{2}-c_{2}, \\
& g_{2}=-2 a^{2}\left(b^{+}+b\right)+c_{1}\left(-2 a^{2}\right)-c_{3}, \text { etc. }
\end{aligned}
$$

Here $\left\{c_{\ell}\right\}_{\ell \in \mathbb{N}}$ denote undetermined summation constants which naturally arise when solving (2.4)-(2.6).

Subsequently, it will also be useful to work with the corresponding homogeneous coefficients $\hat{f}_{j}$ and $\hat{g}_{j}$, defined by vanishing of the constants $c_{k}, k \in \mathbb{N}$,

$$
\begin{array}{ll}
\hat{f}_{0}=1, & \hat{f}_{\ell}=\left.f_{\ell}\right|_{c_{k}=0, k=1, \ldots, \ell}, \quad \ell \in \mathbb{N}, \\
\hat{g}_{0}=0, & \hat{g}_{\ell}=\left.g_{\ell}\right|_{c_{k}=0, k=1, \ldots, \ell+1}, \quad \ell \in \mathbb{N}_{0} .
\end{array}
$$

Hence,

$$
f_{\ell}=\sum_{k=0}^{\ell} c_{\ell-k} \hat{f}_{k}, \quad g_{\ell}=\sum_{k=1}^{\ell} c_{\ell-k} \hat{g}_{k}-c_{\ell+1}, \quad \ell \in \mathbb{N}_{0},
$$

introducing

$$
c_{0}=1
$$


Next we define difference expressions $P_{2 p+2}$ of order $2 p+2$ by

$$
P_{2 p+2}=-L^{p+1}+\sum_{\ell=0}^{p}\left(g_{\ell}+2 a f_{\ell} S^{+}\right) L^{p-\ell}+f_{p+1}, \quad p \in \mathbb{N}_{0} .
$$

Introducing the corresponding homogeneous difference expressions $\widehat{P}_{2 p+2}$ defined by

$$
\widehat{P}_{2 \ell+2}=\left.P_{2 \ell+2}\right|_{c_{k}=0, k=1, \ldots, \ell}, \quad \ell \in \mathbb{N}_{0}
$$

one finds

$$
P_{2 p+2}=\sum_{\ell=0}^{p} c_{p-\ell} \widehat{P}_{2 \ell+2}
$$

Using the recursion relations (2.4)-(2.6), the commutator of $P_{2 p+2}$ and $L$ can be explicitly computed and one obtains

$$
\begin{aligned}
{\left[P_{2 p+2}, L\right]=} & -a\left(g_{p}^{+}+g_{p}+f_{p+1}^{+}+f_{p+1}-2 b^{+} f_{p}^{+}\right) S^{+} \\
& +2\left(-b\left(g_{p}+f_{p+1}\right)+a^{2} f_{p}^{+}-\left(a^{-}\right)^{2} f_{p}^{-}+b^{2} f_{p}\right) \\
& -a^{-}\left(g_{p}+g_{p}^{-}+f_{p+1}+f_{p+1}^{-}-2 b f_{p}\right) S^{-}, \quad p \in \mathbb{N}_{0} .
\end{aligned}
$$

In particular, $\left(L, P_{2 p+2}\right)$ represents the celebrated Lax pair of the Toda hierarchy. Varying $p \in \mathbb{N}_{0}$, the stationary Toda hierarchy is then defined in terms of the vanishing of the commutator of $P_{2 p+2}$ and $L$ in (2.14), that is,

$$
\left[P_{2 p+2}, L\right]=\mathrm{s}_{-} \mathrm{Tl}_{p}(a, b)=0, \quad p \in \mathbb{N}_{0} .
$$

Thus one finds

$$
\begin{array}{r}
g_{p}+g_{p}^{-}+f_{p+1}+f_{p+1}^{-}-2 b f_{p}=0, \\
-b\left(g_{p}+f_{p+1}\right)+a^{2} f_{p}^{+}-\left(a^{-}\right)^{2} f_{g}^{-}+b^{2} f_{p}=0 .
\end{array}
$$

Using (2.5) with $j=p$ one concludes that (2.16) reduces to

$$
f_{p+1}=f_{p+1}^{-},
$$

that is, $f_{p+1}$ is a lattice constant. Similarly, one infers by subtracting $b$ times (2.16) from twice (2.17) and using (2.6) with $j=p$, that $g_{p+1}$ is a lattice constant as well, that is,

$$
g_{p+1}=g_{p+1}^{-}
$$


Equations (2.18) and (2.19) give rise to the stationary Toda hierarchy, which is introduced as follows

$$
\mathrm{s}^{-} \mathrm{Tl}_{p}(a, b)=\left(\begin{array}{c}
f_{p+1}^{+}-f_{p+1} \\
g_{p+1}-g_{p+1}^{-}
\end{array}\right)=0, \quad p \in \mathbb{N}_{0}
$$

Explicitly,

$$
\begin{aligned}
\mathrm{s}-\mathrm{Tl}_{0}(a, b)= & \left(\begin{array}{c}
b^{+}-b \\
2\left(\left(a^{-}\right)^{2}-a^{2}\right)
\end{array}\right)=0 \\
\mathrm{~s}-\mathrm{Tl}_{1}(a, b)= & \left(\begin{array}{c}
\left(a^{+}\right)^{2}-\left(a^{-}\right)^{2}+\left(b^{+}\right)^{2}-b^{2} \\
2\left(a^{-}\right)^{2}\left(b+b^{-}\right)-2 a^{2}\left(b^{+}+b\right)
\end{array}\right) \\
& +c_{1}\left(\begin{array}{c}
b^{+}-b \\
2\left(\left(a^{-}\right)^{2}-a^{2}\right)
\end{array}\right)=0, \text { etc. }
\end{aligned}
$$

represent the first few equations of the stationary Toda hierarchy. By definition, the set of solutions of $(2.20)$, with $p$ ranging in $\mathbb{N}_{0}$ and $c_{\ell} \in \mathbb{C}, \ell \in \mathbb{N}$, represents the class of algebro-geometric Toda solutions.

In the following we will frequently assume that $a, b$ satisfy the $p$ th stationary Toda system. By this we mean it satisfies one of the $p$ th stationary Toda equations after a particular choice of summation constants $c_{\ell} \in \mathbb{C}$, $\ell=1, \ldots, p, p \in \mathbb{N}$, has been made.

In accordance with our notation introduced in (2.8) and (2.12), the corresponding homogeneous stationary Toda equations are defined by

$$
\mathrm{s}-\widehat{\mathrm{Tl}}_{p}(a, b)=\mathrm{s}-\left.\mathrm{Tl}_{p}(a, b)\right|_{c_{\ell}=0, \ell=1, \ldots, p}=0, \quad p \in \mathbb{N}_{0}
$$

Next, we introduce polynomials $F_{p}$ and $G_{p+1}$ of degree $p$ and $p+1$, with respect to the spectral parameter $z \in \mathbb{C}$ by

$$
\begin{aligned}
F_{p}(z) & =\sum_{\ell=0}^{p} f_{p-\ell} z^{\ell}=\sum_{\ell=0}^{p} c_{p-\ell} \widehat{F}_{\ell}(z) \\
G_{p+1}(z) & =-z^{p+1}+\sum_{\ell=0}^{p} g_{p-\ell} z^{\ell}+f_{p+1}=\sum_{\ell=1}^{p+1} c_{p+1-\ell} \widehat{G}_{\ell}(z)
\end{aligned}
$$

with $\widehat{F}_{\ell}$ and $\widehat{G}_{\ell}$ denoting the corresponding homogeneous polynomials de- 
fined by

$$
\begin{aligned}
& \widehat{F}_{0}(z)=F_{0}(z)=1, \\
& \widehat{F}_{\ell}(z)=\left.F_{\ell}(z)\right|_{c_{k}=0, k=1, \ldots, \ell}=\sum_{k=0}^{\ell} \hat{f}_{\ell-k} z^{k}, \quad \ell \in \mathbb{N}_{0}, \\
& \widehat{G}_{0}(z)=\left.G_{0}(z)\right|_{c_{1}=0}=0, \quad \widehat{G}_{1}(z)=G_{1}(z)=-z-b, \\
& \widehat{G}_{\ell+1}(z)=\left.G_{\ell+1}(z)\right|_{c_{k}=0, k=1, \ldots, \ell}=-z^{\ell+1}+\sum_{k=0}^{\ell} \hat{g}_{\ell-k} z^{k}+\hat{f}_{\ell+1}, \quad \ell \in \mathbb{N} .
\end{aligned}
$$

Explicitly, one obtains

$$
\begin{aligned}
& F_{0}=1, \\
& F_{1}=z+b+c_{1}, \\
& F_{2}=z^{2}+b z+a^{2}+\left(a^{-}\right)^{2}+b^{2}+c_{1}(z+b)+c_{2}, \text { etc. } \\
& G_{1}=-z+b \\
& G_{2}=-z^{2}+\left(a^{-}\right)^{2}-a^{2}+b^{2}+c_{1}(-z+b), \text { etc. }
\end{aligned}
$$

Next, we study the restriction of the difference expression $P_{2 p+2}$ to the two-dimensional kernel (i.e., the formal null space in an algebraic sense as opposed to the functional analytic one) of $(L-z)$. More precisely, let

$$
\operatorname{ker}(L-z)=\{\psi: \mathbb{Z} \rightarrow \mathbb{C} \cup\{\infty\} \mid(L-z) \psi=0\} .
$$

Then (2.11) implies

$$
\left.P_{2 p+2}\right|_{\operatorname{ker}(L-z)}=\left.\left(2 a F_{p}(z) S^{+}+G_{p+1}(z)\right)\right|_{\operatorname{ker}(L-z)} .
$$

Therefore, the Lax relation (2.15) becomes

$$
\begin{aligned}
2\left(z-b^{+}\right) F_{p}^{+}-2(z-b) F_{p}+G_{p+1}^{+}-G_{p+1}^{-} & =0, \\
2 a^{2} F_{p}^{+}-2\left(a^{-}\right)^{2} F_{p}^{-}+(z-b)\left(G_{p+1}-G_{p+1}^{-}\right) & =0 .
\end{aligned}
$$

Additional manipulations yield

$$
\begin{array}{r}
2(z-b) F_{p}+G_{p+1}+G_{p+1}^{-}=0, \\
(z-b)^{2} F_{p}+(z-b) G_{p+1}+a^{2} F_{p}^{+}-\left(a^{-}\right)^{2} F_{p}^{-}=0 .
\end{array}
$$

Indeed, adding $G_{p+1}-G_{p+1}$ to the left-hand side of (2.30) (neglecting a trivial summation constant) yields (2.32) and inserting (2.30) into (2.31) then implies (2.33). Varying $p \in \mathbb{N}_{0}$, equations (2.32), (2.33) provide an alternative description of the stationary Toda hierarchy. 
Combining equations (2.31) and (2.32) one concludes that the quantity

$$
R_{2 p+2}(z)=G_{p+1}(z, n)^{2}-4 a(n)^{2} F_{p}(z, n) F_{p}^{+}(z, n)
$$

is a lattice constant, and hence depends on $z$ only. Thus, one can write

$$
R_{2 p+2}(z)=\prod_{m=0}^{2 p+1}\left(z-E_{m}\right), \quad\left\{E_{m}\right\}_{m=0}^{2 p+1} \subset \mathbb{C} .
$$

One can decouple (2.32) and (2.33) to obtain separate equations for $F_{p}$ and $G_{p+1}$. For instance, computing $G_{p+1}$ from (2.33) and inserting the result into (2.32) yields the following linear difference equation for $F_{p}$

$$
\begin{aligned}
0= & (z-b)^{2}\left(z-b^{-}\right) F_{p}-\left(z-b^{-}\right)^{2}(z-b) F_{p}^{-}+ \\
& +\left(\left(a^{-}\right)^{2} F_{p}^{-}-a^{2} F_{p}^{+}\right)\left(z-b^{-}\right)+\left(\left(a^{--}\right)^{2} F_{p}^{--}-\left(a^{-}\right)^{2} F_{p}\right)(z-b) .
\end{aligned}
$$

Similarly, insertion of (2.33) into (2.34) permits one to eliminate $G_{p+1}$ and results in the following nonlinear difference equation for $F_{p}$,

$$
\begin{gathered}
(z-b)^{4} F_{p}^{2}-2 a^{2}(z-b)^{2} F_{p} F_{p}^{+}-2\left(a^{-}\right)^{2}(z-b)^{2} F_{p} F_{p}^{-}+a^{4}\left(F_{p}^{+}\right)^{2} \\
+\left(a^{-}\right)^{4}\left(F_{p}^{-}\right)^{2}-2 a^{2}\left(a^{-}\right)^{2} F_{p}^{+} F_{p}^{-}=(z-b)^{2} R_{2 p+2}(z) .
\end{gathered}
$$

On the other hand, computing $F_{p}$ in terms of $G_{p+1}$ and $G_{p+1}^{+}$using $(2.32)$ and inserting the result into (2.33) yields the following linear difference equation for $G_{p+1}$

$$
\begin{aligned}
& a^{2}\left(z-b^{-}\right)\left(G_{p+1}^{+}+G_{p+1}\right)-\left(a^{-}\right)^{2}\left(z-b^{+}\right)\left(G_{p+1}^{-}+G_{p+1}^{--}\right) \\
& \quad+\left(z-b^{-}\right)(z-b)\left(z-b^{+}\right)\left(G_{p+1}^{-}-G_{p+1}\right)=0 .
\end{aligned}
$$

Finally, inserting the result for $F_{p}$ into (2.34) yields the following nonlinear difference equation for $G_{p+1}$

$$
\begin{aligned}
& (z-b)\left(z-b^{+}\right) G_{p+1}^{2}-a^{2}\left(G_{p+1}^{-}+G_{p+1}\right)\left(G_{p+1}+G_{p+1}^{+}\right) \\
& =(z-b)\left(z-b^{+}\right) R_{2 p+2} .
\end{aligned}
$$

Equations (2.37) and (2.39) can be used to derive nonlinear recursion relations for the homogeneous coefficients $\hat{f}_{\ell}$ and $\hat{g}_{\ell}$ (i.e., the ones satisfying (2.8) in the case of vanishing summation constants) as proved in Theorem C.1 in Appendix C. This has interesting applications to the asymptotic expansion of the Green's function of $L$ with respect to the spectral parameter. In addition, as proven in Theorem C.1, (2.37) leads to an explicit determination of the summation constants $c_{1}, \ldots, c_{p}$ in

$$
\mathrm{s}-\mathrm{Tl}_{p}(a, b)=0, \quad p \in \mathbb{N}_{0},
$$

in terms of the zeros $E_{0}, \ldots, E_{2 p+1}$ of the associated polynomial $R_{2 p+2}$ in (2.35). 
In fact, one can prove (cf. Theorem C.1) that

$$
c_{k}=c_{k}(\underline{E}), \quad k=1, \ldots, p,
$$

where

$$
\begin{gathered}
c_{k}(\underline{E})=-\sum_{\substack{j_{0}, \ldots, j_{2 p+1}=0 \\
j_{0}+\cdots+j_{2 p+1}=k}}^{k} \frac{\left(2 j_{0}\right) ! \cdots\left(2 j_{2 p+1}\right) !}{2^{2 k}\left(j_{0} !\right)^{2} \cdots\left(j_{2 p+1} !\right)^{2}\left(2 j_{0}-1\right) \cdots\left(2 j_{2 p+1}-1\right)} \\
\times E_{0}^{j_{0}} \cdots E_{2 p+1}^{j_{2 p+1}}, \quad k=1, \ldots, p,
\end{gathered}
$$

are symmetric functions of $\underline{E}=\left(E_{0}, \ldots, E_{2 p+1}\right)$.

We emphasize that the result (2.29) is valid independently of whether or not $P_{2 p+2}$ and $L$ commute. However, the fact that the two difference expressions $P_{2 p+2}$ and $L$ commute implies the existence of an algebraic relationship between them. This gives rise to the Burchnall-Chaundy polynomial for the Toda hierarchy first discussed in the discrete context by Nauman [28], [29].

Theorem 2.2. Assume Hypothesis 2.1, fix $p \in \mathbb{N}_{0}$ and suppose that $P_{2 p+2}$ and $L$ commute, $\left[P_{2 p+2}, L\right]=0$, or equivalently, assume that $\mathrm{s}-\mathrm{Tl}_{p}(a, b)=0$. Then $L$ and $P_{2 p+2}$ satisfy an algebraic relationship of the type (cf. (2.35))

$$
\begin{aligned}
& \mathcal{F}_{p}\left(L, P_{2 p+2}\right)=P_{2 p+2}^{2}-R_{2 p+2}(L)=0, \\
& R_{2 p+2}(z)=\prod_{m=0}^{2 p+1}\left(z-E_{m}\right), \quad z \in \mathbb{C} .
\end{aligned}
$$

The expression $\mathcal{F}_{p}\left(L, P_{2 p+2}\right)$ is called the Burchnall-Chaundy polynomial of the Lax pair $\left(L, P_{2 p+2}\right)$ and it will be used in Section 3 to introduce the underlying hyperelliptic curve associated with the stationary Toda system s- $\mathrm{Tl}_{p}(a, b)=0$ (cf. (3.1)).

Next we turn to the time-dependent Toda hierarchy. For that purpose the functions $a$ and $b$ are now considered as functions of both the lattice point and time. For each equation in the hierarchy, that is, for each $p$, we introduce a deformation (time) parameter $t_{p} \in \mathbb{R}$ in $a, b$, replacing $a(n), b(n)$ by $a\left(n, t_{p}\right), b\left(n, t_{p}\right)$. The second-order difference expression $L$ (cf. (2.2)) now reads

$$
L\left(t_{p}\right)=a\left(\cdot, t_{p}\right) S^{+}+a^{-}\left(\cdot, t_{p}\right) S^{-}+b\left(\cdot, t_{p}\right) .
$$

The quantities $\left\{f_{\ell}\right\}_{\ell \in \mathbb{N}_{0}},\left\{g_{\ell}\right\}_{\ell \in \mathbb{N}_{0}}$, and $P_{2 p+2}, p \in \mathbb{N}_{0}$ are still defined by (2.4)-(2.6) and (2.11), respectively. The time-dependent Toda hierarchy is then obtained by imposing the Lax commutator equations

$$
L_{t_{p}}\left(t_{p}\right)-\left[P_{2 p+2}\left(t_{p}\right), L\left(t_{p}\right)\right]=0, \quad t_{p} \in \mathbb{R}
$$


varying $p \in \mathbb{N}_{0}$. Relation (2.45) implies

$$
\begin{aligned}
& \left(a_{t_{p}}+a\left(g_{p}^{+}+g_{p}+f_{p+1}^{+}+f_{p+1}-2 b^{+} f_{p}^{+}\right)\right) S^{+} \\
& \quad-\left(-b_{t_{p}}+2\left(-b\left(g_{p}+f_{p+1}\right)+a^{2} f_{p}^{+}-\left(a^{-}\right)^{2} f_{p}^{-}+b^{2} f_{p}\right)\right) \\
& \quad+\left(a_{t_{p}}+a\left(g_{p}^{+}+g_{p}+f_{p+1}^{+}+f_{p+1}-2 b^{+} f_{p}^{+}\right)\right)^{-} S^{-}=0 .
\end{aligned}
$$

Applying the same method we used to derive (2.18) and (2.19) one concludes

$$
\begin{aligned}
0= & L_{t_{p}}-\left[P_{2 p+2}, L\right] \\
= & \left(a_{t_{p}}-a\left(f_{p+1}^{+}-f_{p+1}\right)\right) S^{+}-\left(-b_{t_{p}}-g_{p+1}+g_{p+1}^{-}\right) \\
& +\left(a_{t_{p}}-a\left(f_{p+1}^{+}-f_{p+1}\right)\right)^{-} S^{-} .
\end{aligned}
$$

Varying $p \in \mathbb{N}_{0}$, the collection of evolution equations

$$
\mathrm{Tl}_{p}(a, b)=\left(\begin{array}{c}
a_{t_{p}}-a\left(f_{p+1}^{+}-f_{p+1}\right) \\
b_{t_{p}}+g_{p+1}-g_{p+1}^{-}
\end{array}\right)=0, \quad\left(n, t_{p}\right) \in \mathbb{Z} \times \mathbb{R}, p \in \mathbb{N}_{0},
$$

then defines the time-dependent Toda hierarchy. Explicitly,

$$
\begin{aligned}
\mathrm{Tl}_{0}(a, b)= & \left(\begin{array}{c}
a_{t_{0}}-a\left(b^{+}-b\right) \\
b_{t_{0}}-2\left(a^{2}-\left(a^{-}\right)^{2}\right)
\end{array}\right)=0, \\
\mathrm{Tl}_{1}(a, b)= & \left(\begin{array}{c}
a_{t_{1}}-a\left(\left(a^{+}\right)^{2}-\left(a^{-}\right)^{2}+\left(b^{+}\right)^{2}-b^{2}\right) \\
b_{t_{1}}+2\left(a^{-}\right)^{2}\left(b+b^{-}\right)-2 a^{2}\left(b^{+}+b\right)
\end{array}\right) \\
& +c_{1}\left(\begin{array}{c}
-a\left(b^{+}-b\right) \\
-2\left(a^{2}-\left(a^{-}\right)^{2}\right)
\end{array}\right)=0, \text { etc., }
\end{aligned}
$$

represent the first few equations of the time-dependent Toda hierarchy. The system of equations, $\mathrm{Tl}_{0}(a, b)=0$, is of course the Toda system.

The corresponding homogeneous Toda equations obtained by taking all summation constants equal to zero, $c_{\ell}=0, \ell=1, \ldots, p$, are then denoted by

$$
\widehat{\mathrm{Tl}}_{p}(a, b)=\left.\mathrm{Tl}_{p}(a, b)\right|_{c_{\ell}=0, \ell=1, \ldots, p} .
$$

Restricting the Lax relation (2.45) to the kernel $\operatorname{ker}(L-z)$ one finds that

$$
\begin{aligned}
0= & \left.\left(L_{t_{p}}-\left[P_{2 p+2}, L\right]\right)\right|_{\operatorname{ker}(L-z)}=\left.\left(L_{t_{p}}+(L-z) P_{2 p+2}\right)\right|_{\operatorname{ker}(L-z)} \\
= & \left(a\left(\frac{a_{t_{p}}}{a}-\frac{a_{t_{p}}^{-}}{a^{-}}+2\left(z-b^{+}\right) F_{p}^{+}-2(z-b) F_{p}+G_{p+1}^{+}-G_{p+1}^{-}\right) S^{+}\right. \\
& +\left(b_{t_{p}}+(z-b) \frac{a_{t_{p}}^{-}}{a^{-}}+2\left(a^{-}\right)^{2} F_{p}^{-}-2 a^{2} F_{p}^{+}\right. \\
& \left.\left.+(z-b)\left(G_{p+1}^{-}-G_{p+1}\right)\right)\right)\left.\right|_{\operatorname{ker}(L-z)} .
\end{aligned}
$$


Hence one obtains

$$
\begin{aligned}
& \frac{a_{t_{p}}}{a}-\frac{a_{t_{p}}^{-}}{a^{-}}=-2\left(z-b^{+}\right) F_{p}^{+}+2(z-b) F_{p}+G_{p+1}^{-}-G_{p+1}^{+}, \\
& b_{t_{p}}=-(z-b) \frac{a_{t_{p}}^{-}}{a^{-}}-2\left(a^{-}\right)^{2} F_{p}^{-}+2 a^{2} F_{p}^{+}-(z-b)\left(G_{p+1}^{-}-G_{p+1}\right) .
\end{aligned}
$$

Further manipulations then yield,

$$
\begin{aligned}
a_{t_{p}} & =-a\left(2\left(z-b^{+}\right) F_{p}^{+}+G_{p+1}^{+}+G_{p+1}\right), \\
b_{t_{p}} & =2\left((z-b)^{2} F_{p}+(z-b) G_{p+1}+a^{2} F_{p}^{+}-\left(a^{-}\right)^{2} F_{p}^{-}\right) .
\end{aligned}
$$

Indeed, (2.55) follows by adding $G_{p+1}-G_{p+1}$ to (2.53) (neglecting a trivial summation constant), and an insertion of (2.55) into (2.54) implies (2.56). Varying $p \in \mathbb{N}_{0}$, equations (2.55) and (2.56) provide an alternative description of the time-dependent Toda hierarchy.

Remark 2.3. From (2.4)-(2.6) and (2.23), (2.24) one concludes that the coefficient a enters quadratically in $F_{p}$ and $G_{p+1}$, and hence the Toda hierarchy (2.48) (respectively (2.20)) is invariant under the substitution

$$
a \rightarrow a_{\varepsilon}=\{\varepsilon(n) a(n)\}_{n \in \mathbb{Z}}, \quad \varepsilon(n) \in\{1,-1\}, n \in \mathbb{Z} .
$$

We conclude this section by pointing out an alternative construction of the Toda hierarchy using a zero-curvature approach instead of Lax pairs $\left(L, P_{2 p+2}\right)$. To this end one defines the $2 \times 2$ matrices

$$
\begin{aligned}
U(z) & =\left(\begin{array}{cc}
0 & 1 \\
-a^{-} / a & (z-b) / a
\end{array}\right), \\
V_{p+1}(z) & =\left(\begin{array}{cc}
G_{p+1}^{-}(z) & 2 a^{-} F_{p}^{-}(z) \\
-2 a^{-} F_{p}(z) & 2(z-b) F_{p}(z)+G_{p+1}(z)
\end{array}\right), \quad p \in \mathbb{N}_{0} .
\end{aligned}
$$

Then the stationary part of this section can equivalently be based on the zero-curvature equation

$$
\begin{aligned}
0 & =U V_{p+1}-V_{p+1}^{+} U \\
& =\frac{2}{a}\left(\begin{array}{cc}
a^{-}\left(\left(z-b^{+}\right) F_{p}^{+}-(z-b) F_{p}\right. & a^{2} F_{p}^{+}-\left(a^{-}\right)^{2} F_{p}^{-} \\
\left.+2^{-1}\left(G_{p+1}^{+}-G_{p+1}^{-}\right)\right) & +2^{-1}(z-b)\left(G_{p+1}-G_{p+1}^{+}\right) \\
& +(z-b)^{2} F_{p}-\left(z-b^{+}\right)(z-b) F_{p}^{+}
\end{array}\right) .
\end{aligned}
$$

Thus, one obtains (2.30) from the $(2,1)$-entry in (2.60). Insertion of $(2.30)$ into the $(2,2)$-entry of $(2.60)$ then yields $(2.31)$. Thus, one also obtains (2.32) and hence the $(2,2)$-entry of $V_{p+1}$ in (2.59) simplifies to

$$
V_{p+1,2,2}(z)=-G_{p+1}^{-}(z)
$$


in the stationary case. Since $\operatorname{det}(U(z, n))=a^{-}(n) / a(n) \neq 0, n \in \mathbb{Z}$, the zero-curvature equation (2.60) yields that $\operatorname{det}\left(V_{p+1}(z, n)\right)$ is a lattice constant (i.e., independent of $n \in \mathbb{Z}$ ). The Burchnall-Chaundy polynomial $\mathcal{F}_{p}(y, z)$ (cf. (2.43) and especially, the hyperelliptic curve (3.1)) is then obtained from the characteristic equation of $V_{p+1}(z)$ by

$$
\begin{aligned}
\operatorname{det} & \left(y I_{2}-V_{p+1}(z, n)\right) \\
= & y^{2}+\operatorname{det}\left(V_{p+1}(z, n)\right) \\
= & y^{2}-G_{p-1}^{-}(z, n)^{2}+4 a^{-}(n)^{2} F_{p}^{-}(z, n) F_{p}(z, n) \\
= & y^{2}-R_{2 p+2}(z)=0,
\end{aligned}
$$

using (2.61). (Here $I_{2}$ denotes the identity matrix in $\mathbb{C}^{2}$.) Similarly, the time-dependent part (2.44)-(2.56) can equivalently be developed from the zero-curvature equation

$$
\begin{aligned}
0 & =U_{t_{p}}+U V_{p+1}-V_{p+1}^{+} U \\
0 & =\frac{1}{a}\left(\begin{array}{cc}
a^{-}\left(\left(a_{t_{p}} / a\right)-\left(a_{t_{p}}^{-} / a^{-}\right)\right) & -b_{t_{p}}-(z-b)\left(a_{t_{p}} / a\right) \\
+a^{-}\left(2\left(z-b^{+}\right) F_{p}^{+}-2(z-b) F_{p}\right. & +2 a^{2} F_{p}^{+}-2\left(a^{-}\right)^{2} F_{p}^{-} \\
\left.+\left(G_{p+1}^{+}-G_{p+1}^{-}\right)\right) & +(z-b)\left(G_{p+1}-G_{p+1}^{+}\right) \\
& +2(z-b)^{2} F_{p}-2\left(z-b^{+}\right)(z-b) F_{p}^{+}
\end{array}\right) .
\end{aligned}
$$

The $(2,1)$-entry in $(2.63)$ yields $(2.53)$, and inserting $(2.53)$ into the $(2,2)$ entry of (2.63) yields (2.54) and hence also the basic equations defining the time-dependent Toda hierarchy in (2.55), (2.56).

\section{Properties of stationary algebro-geometric solutions of the Toda hierarchy}

In this section we present a quick review of properties of algebro-geometric solutions of the stationary Toda hierarchy. Since this material is standard we omit all proofs and just refer to [6] (cf. also [14, Sect. 1.3], [32, Chs. 8, 9]) for detailed presentations and an extensive list of references to the literature.

For the notation employed in connection with elementary concepts in algebraic geometry (more precisely, the theory of compact Riemann surfaces), we refer to Appendix A.

Returning to Theorem 2.2, we note that (2.43) naturally leads to the hyperelliptic curve $\mathcal{K}_{p}$ of genus $p \in \mathbb{N}_{0}$, where

$$
\begin{aligned}
& \mathcal{K}_{p}: \mathcal{F}_{p}(z, y)=y^{2}-R_{2 p+2}(z)=0 \\
& R_{2 p+2}(z)=\prod_{m=0}^{2 p+1}\left(z-E_{m}\right), \quad\left\{E_{m}\right\}_{m=0}^{2 p+1} \subset \mathbb{C} .
\end{aligned}
$$


Throughout this section we make the following assumption:

\section{Hypothesis 3.1. Suppose that}

$$
a, b \in \mathbb{C}^{\mathbb{Z}} \text { and } a(n) \neq 0 \text { for all } n \in \mathbb{Z} \text {. }
$$

In addition, assume that the hyperelliptic curve $\mathcal{K}_{p}$ in (3.1) is nonsingular, that is, suppose that

$$
E_{m} \neq E_{m^{\prime}} \text { for } m \neq m^{\prime}, m, m^{\prime}=0, \ldots, 2 p+1 \text {. }
$$

The curve $\mathcal{K}_{p}$ is compactified by joining two points $P_{\infty_{ \pm}}, P_{\infty_{+}} \neq P_{\infty_{-}}$, at infinity. For notational simplicity, the resulting curve is still denoted by $\mathcal{K}_{p}$. Points $P$ on $\mathcal{K}_{p} \backslash\left\{P_{\infty_{+}}, P_{\infty_{-}}\right\}$are represented as pairs $P=(z, y)$, where $y(\cdot)$ is the meromorphic function on $\mathcal{K}_{p}$ satisfying $\mathcal{F}_{p}(z, y)=0$. The complex structure on $\mathcal{K}_{p}$ is then defined in the usual way, see Appendix A. Hence, $\mathcal{K}_{p}$ becomes a two-sheeted hyperelliptic Riemann surface of genus $p \in \mathbb{N}_{0}$ in a standard manner.

We also emphasize that by fixing the curve $\mathcal{K}_{p}$ (i.e., by fixing $E_{0}, \ldots, E_{2 p+1}$ ), the summation constants $c_{1}, \ldots, c_{p}$ in the corresponding stationary $\mathrm{s}-\mathrm{Tl}_{p}$ equation are uniquely determined as is clear from (2.41) and (2.42), which establish the summation constants $c_{k}$ as symmetric functions of $E_{0}, \ldots, E_{2 p+1}$.

For notational simplicity we will usually tacitly assume that $p \in \mathbb{N}$. The trivial case $p=0$, which leads to $a(n)^{2}=\left(E_{1}-E_{0}\right)^{2} / 16, b(n)=\left(E_{0}+E_{1}\right) / 2$, $n \in \mathbb{Z}$, is of no interest to us in this paper.

In the following, the zeros ${ }^{2}$ of the polynomial $F_{p}(\cdot, n)$ (cf. $\left.(2.23)\right)$ will play a special role. We denote them by $\left\{\mu_{j}(n)\right\}_{j=1}^{p}$ and write

$$
F_{p}(z, n)=\prod_{j=1}^{p}\left(z-\mu_{j}(n)\right) .
$$

The next step is crucial; it permits us to "lift" the zeros $\mu_{j}$ of $F_{p}$ from $\mathbb{C}$ to the curve $\mathcal{K}_{p}$. From (2.34) and (3.4) one infers

$$
R_{2 p+2}(z)-G_{p+1}(z)^{2}=0, \quad z \in\left\{\mu_{j}, \mu_{k}^{+}\right\}_{j, k=1, \ldots, p} .
$$

We now introduce $\left\{\hat{\mu}_{j}(n)\right\}_{j=1, \ldots, p} \subset \mathcal{K}_{p}$ by

$$
\hat{\mu}_{j}(n)=\left(\mu_{j}(n),-G_{p+1}\left(\mu_{j}(n), n\right)\right) \in \mathcal{K}_{p}, \quad j=1, \ldots, p, n \in \mathbb{Z} .
$$

\footnotetext{
${ }^{2}$ If $a, b \in \ell^{\infty}(\mathbb{Z})$, these zeros are the Dirichlet eigenvalues of a bounded operator on $\ell^{2}(\mathbb{Z})$ associated with the difference expression $L$ and a Dirichlet boundary condition at $n \in \mathbb{Z}$.
} 
Next, we recall equation (2.34) and define the fundamental meromorphic function $\phi(\cdot, n)$ on $\mathcal{K}_{p}$ by

$$
\begin{aligned}
\phi(P, n) & =\frac{y-G_{p+1}(z, n)}{2 a(n) F_{p}(z, n)} \\
& =\frac{-2 a(n) F_{p}(z, n+1)}{y+G_{p+1}(z, n)}, \\
P & =(z, y) \in \mathcal{K}_{p}, n \in \mathbb{Z},
\end{aligned}
$$

with divisor $(\phi(\cdot, n))$ of $\phi(\cdot, n)$ given by

$$
(\phi(\cdot, n))=\mathcal{D}_{P_{\infty_{+}} \underline{\hat{\mu}}(n+1)}-\mathcal{D}_{P_{\infty_{-}} \underline{\hat{\mu}}(n)},
$$

using (3.4) and (3.6). Here we abbreviated

$$
\underline{\hat{\mu}}=\left\{\hat{\mu}_{1}, \ldots, \hat{\mu}_{p}\right\} \in \operatorname{Sym}^{p}\left(\mathcal{K}_{p}\right)
$$

(cf. the notation introduced in Appendix A). We note that several $\mu_{j}(n)$ may be equal for a given lattice point $n \in \mathbb{Z}$. Moreover, since $-G_{p+1}\left(\mu_{j}(n), n\right)$ takes on the same value for all coinciding zeros $\mu_{j}(n)$, no finite special divisors $\mathcal{D}_{\hat{\mu}(n)}$ can ever arise in $\phi$ (cf. also Lemma 3.4).

The stationary Baker-Akhiezer function $\psi\left(\cdot, n, n_{0}\right)$ on $\mathcal{K}_{p} \backslash\left\{P_{\infty_{ \pm}}\right\}$is then defined in terms of $\phi(\cdot, n)$ by

$$
\begin{aligned}
& \psi\left(P, n, n_{0}\right)= \begin{cases}\prod_{m=n_{0}}^{n-1} \phi(P, m) & \text { for } n \geq n_{0}+1, \\
1 & \text { for } n=n_{0}, \\
\prod_{m=n}^{n_{0}-1} \phi(P, m)^{-1} & \text { for } n \leq n_{0}-1,\end{cases} \\
& P \in \mathcal{K}_{p} \backslash\left\{P_{\infty_{ \pm}}\right\},\left(n, n_{0}\right) \in \mathbb{Z}^{2},
\end{aligned}
$$

with divisor $\left(\psi\left(\cdot, n, n_{0}\right)\right)$ of $\psi\left(P, n, n_{0}\right)$ given by

$$
\left(\psi\left(\cdot, n, n_{0}\right)\right)=\mathcal{D}_{\underline{\hat{\mu}}(n)}-\mathcal{D}_{\underline{\hat{\mu}}\left(n_{0}\right)}+\left(n-n_{0}\right)\left(\mathcal{D}_{P_{\infty_{+}}}-\mathcal{D}_{P_{\infty_{-}}}\right) .
$$

For future purposes we also introduce the following Baker-Akhiezer vector,

$$
\Psi\left(P, n, n_{0}\right)=\left(\begin{array}{c}
\psi^{-}\left(P, n, n_{0}\right) \\
\psi\left(P, n, n_{0}\right)
\end{array}\right), \quad P \in \mathcal{K}_{p} \backslash\left\{P_{\infty_{ \pm}}\right\},\left(n, n_{0}\right) \in \mathbb{Z}^{2} .
$$

Basic properties of $\phi, \psi$, and $\Psi$ are summarized in the following result. We abbreviate by

$$
W(f, g)=a\left(f g^{+}-f^{+} g\right)
$$

the Wronskian of two complex-valued sequences $f$ and $g$, and denote $P^{*}=$ $(z,-y)$ for $P=(z, y) \in \mathcal{K}_{p}$. 
Lemma 3.2. Assume Hypothesis 3.1 and suppose that $a, b$ satisfy the pth stationary Toda system (2.20). Moreover, let $P=(z, y) \in \mathcal{K}_{p} \backslash\left\{P_{\infty_{ \pm}}\right\}$and $\left(n, n_{0}\right) \in \mathbb{Z}^{2}$. Then $\phi$ satisfies the Riccati-type equation

$$
a \phi(P)+a^{-} \phi^{-}(P)^{-1}=z-b,
$$

as well as

$$
\begin{aligned}
& \phi(P) \phi\left(P^{*}\right)=\frac{F_{p}^{+}(z)}{F_{p}(z)} \\
& \phi(P)+\phi\left(P^{*}\right)=-\frac{G_{p+1}(z)}{a F_{p}(z)}, \\
& \phi(P)-\phi\left(P^{*}\right)=\frac{y(P)}{a F_{p}(z)} .
\end{aligned}
$$

Moreover, $\psi$ and $\Psi$ satisfy

$$
\begin{aligned}
& (L-z(P)) \psi(P)=0, \quad\left(P_{2 p+2}-y(P)\right) \psi(P)=0, \\
& \Psi^{+}(P)=U(z) \Psi(P), \quad y \Psi(P)=V_{p+1} \Psi(P), \\
& \psi\left(P, n, n_{0}\right) \psi\left(P^{*}, n, n_{0}\right)=\frac{F_{p}(z, n)}{F_{p}\left(z, n_{0}\right)}, \\
& a(n)\left(\psi\left(P, n, n_{0}\right) \psi\left(P^{*}, n+1, n_{0}\right)+\psi\left(P^{*}, n, n_{0}\right) \psi\left(P, n+1, n_{0}\right)\right) \\
& \quad=-\frac{G_{p+1}(z, n)}{F_{p}\left(z, n_{0}\right)}, \\
& W\left(\psi\left(P, \cdot, n_{0}\right), \psi\left(P^{*}, \cdot, n_{0}\right)\right)=-\frac{y(P)}{F_{p}\left(z, n_{0}\right)} .
\end{aligned}
$$

Combining the polynomial recursion approach with (3.4) readily yields trace formulas for the Toda invariants, which are expressions of $a$ and $b$ in terms of the zeros $\mu_{j}$ of $F_{p}$. We introduce the abbreviation,

$$
b^{(k)}(n)=\frac{1}{2} \sum_{m=0}^{2 p+1} E_{m}^{k}-\sum_{j=1}^{p} \mu_{j}^{k}(n), \quad k \in \mathbb{N} .
$$

Lemma 3.3. Assume Hypothesis 3.1 and suppose that $a, b$ satisfy the pth stationary Toda system (2.20). Then,

$$
b(n)=\frac{1}{2} \sum_{m=0}^{2 p+1} E_{m}-\sum_{j=1}^{p} \mu_{j}(n), \quad n \in \mathbb{Z} .
$$


In addition, if for all $n \in \mathbb{Z}, \mu_{j}(n) \neq \mu_{k}(n)$ for $j \neq k, j, k=1, \ldots, p$, then,

$$
\begin{gathered}
a(n)^{2}=\frac{1}{2} \sum_{j=1}^{p} y\left(\hat{\mu}_{j}(n)\right) \prod_{\substack{k=1 \\
k \neq j}}^{p}\left(\mu_{j}(n)-\mu_{k}(n)\right)^{-1} \\
+\frac{1}{4}\left(b^{(2)}(n)-b(n)^{2}\right), \quad n \in \mathbb{Z} .
\end{gathered}
$$

The case where some of the $\mu_{j}$ coincide in (3.26) requires a more elaborate argument that will be presented in Section 4.

Since nonspecial Dirichlet divisors $\mathcal{D}_{\hat{\mu}}$ and the linearization property of the Abel map when applied to $\mathcal{D}_{\hat{\mu}}$ will play a fundamental role later on, we also recall the following facts.

Lemma 3.4. Assume Hypothesis 3.1 and suppose that $a, b$ satisfy the pth stationary Toda system (2.20). Let $\mathcal{D}_{\hat{\mu}}, \underline{\hat{\mu}}=\left\{\hat{\mu}_{1}, \ldots, \hat{\mu}_{p}\right\} \in \operatorname{Sym}^{p}\left(\mathcal{K}_{p}\right)$, be the Dirichlet divisor of degree $p$ associated with $a, b$ defined according to (3.6), that is,

$$
\hat{\mu}_{j}(n)=\left(\mu_{j}(n),-G_{p+1}\left(\mu_{j}(n), n\right)\right) \in \mathcal{K}_{p}, \quad j=1, \ldots, p, n \in \mathbb{Z} .
$$

Then $\mathcal{D}_{\hat{\mu}(n)}$ is nonspecial for all $n \in \mathbb{Z}$. Moreover, the Abel map linearizes the auxiliary divisor $\mathcal{D}_{\underline{\hat{\mu}}}$ in the sense that

$$
\underline{\alpha}_{Q_{0}}\left(\mathcal{D}_{\underline{\hat{\mu}}(n)}\right)=\underline{\alpha}_{Q_{0}}\left(\mathcal{D}_{\underline{\hat{\mu}}\left(n_{0}\right)}\right)-\left(n-n_{0}\right) \underline{A}_{P_{\infty_{-}}}\left(P_{\infty_{+}}\right),
$$

where $Q_{0} \in \mathcal{K}_{p}$ is a given base point.

If in addition, $a, b \in \ell^{\infty}(\mathbb{Z})$, then there exists a constant $C_{\mu}>0$ such that

$$
\left|\mu_{j}(n)\right| \leq C_{\mu}, \quad j=1, \ldots, p, n \in \mathbb{Z} .
$$

Remark 3.5. We note that by construction, the divisors $\mathcal{D}_{\underline{\hat{\mu}}(n)}, n \in \mathbb{Z}$, as introduced in (3.6) are all finite and hence nonspecial by Lemma 3.4. On the other hand, as we will see in the next Section 4, given a nonspecial divisor $\mathcal{D}_{\underline{\hat{\mu}}\left(n_{0}\right)}$, the solution $\mathcal{D}_{\underline{\hat{\mu}}(n)}$ of equation $(3.28)$ may cease to be a finite divisor at some $n \in \mathbb{Z}$.

\section{An algorithm for solving the inverse algebro-geome- tric spectral problem for (non-self-adjoint) Jacobi operators}

The aim of this section is to derive an algorithm that enables one to construct algebro-geometric solutions for the stationary Toda hierarchy for complexvalued initial data. Equivalently, we offer a solution of the inverse algebrogeometric spectral problem for general (non-self-adjoint) Jacobi operators, starting with initial divisors in general complex position. 
Up to the end of Section 3 the material was standard (see [6] and [14, Sect. 1.3], [32, Chs. 8, 9] for details) and based on the assumption that $a, b \in \mathbb{C}^{\mathbb{Z}}$ satisfy the $p$ th stationary Toda system (2.20). Now we embark on the corresponding inverse problem consisting of constructing a solution of (2.20) given certain initial data. More precisely, we seek to construct solutions $a, b \in \mathbb{C}^{\mathbb{Z}}$ satisfying the $p$ th stationary Toda system (2.20) starting from a properly restricted set $\mathcal{M}_{0}$ of finite nonspecial Dirichlet divisor initial data $\mathcal{D}_{\underline{\hat{\mu}}\left(n_{0}\right)}$ at some fixed $n_{0} \in \mathbb{Z}$

$$
\begin{aligned}
\underline{\hat{\mu}}\left(n_{0}\right) & =\left\{\hat{\mu}_{1}\left(n_{0}\right), \ldots, \hat{\mu}_{p}\left(n_{0}\right)\right\} \in \mathcal{M}_{0}, \quad \mathcal{M}_{0} \subset \operatorname{Sym}^{p}\left(\mathcal{K}_{p}\right), \\
\hat{\mu}_{j}\left(n_{0}\right) & =\left(\mu_{j}\left(n_{0}\right),-G_{p+1}\left(\mu_{j}\left(n_{0}\right), n_{0}\right)\right), \quad j=1, \ldots, p .
\end{aligned}
$$

Of course we would like to ensure that the sequences obtained via our algorithm do not blow up. To investigate when this happens, we study the image of our divisors under the Abel map. The key ingredient in our analysis will be (3.28) which yields a linear discrete dynamical system on the Jacobi variety $J\left(\mathcal{K}_{p}\right)$. In particular, we will be led to investigate solutions $\mathcal{D}_{\underline{\underline{\mu}}}$ of the discrete initial value problem

$$
\begin{aligned}
& \underline{\alpha}_{Q_{0}}\left(\mathcal{D}_{\underline{\hat{\mu}}(n)}\right)=\underline{\alpha}_{Q_{0}}\left(\mathcal{D}_{\underline{\hat{\mu}}\left(n_{0}\right)}\right)-\left(n-n_{0}\right) \underline{A}_{P_{\infty_{-}}}\left(P_{\infty_{+}}\right), \\
& \underline{\hat{\mu}}\left(n_{0}\right)=\left\{\hat{\mu}_{1}\left(n_{0}\right), \ldots, \hat{\mu}_{p}\left(n_{0}\right)\right\} \in \operatorname{Sym}^{p}\left(\mathcal{K}_{p}\right),
\end{aligned}
$$

where $Q_{0} \in \mathcal{K}_{p}$ is a given base point. Eventually, we will be interested in solutions $\mathcal{D}_{\underline{\hat{\mu}}}$ of $(4.2)$ with initial data $\mathcal{D}_{\underline{\hat{\mu}}\left(n_{0}\right)}$ satisfying $(4.1)$ and $\mathcal{M}_{0}$ to be specified as in (the proof of) Lemma 4.2.

Before proceeding to develop the stationary Toda algorithm, we briefly analyze the dynamics of (4.2).

Lemma 4.1. Let $\mathcal{D}_{\underline{\hat{\mu}}(n)}$ be defined via (4.2) for some divisor $\mathcal{D}_{\underline{\hat{\mu}}\left(n_{0}\right)} \in \operatorname{Sym}^{p}\left(\mathcal{K}_{p}\right)$. (i) If $\mathcal{D}_{\hat{\mu}(n)}$ is finite and nonspecial and $\mathcal{D}_{\hat{\mu}(n+1)}$ is infinite, then $\mathcal{D}_{\hat{\mu}(n+1)}$ contains $P_{\infty_{+}}$but not $P_{\infty_{-}}$.

(ii) If $\mathcal{D}_{\underline{\hat{\mu}}(n)}$ is nonspecial and $\mathcal{D}_{\underline{\hat{\mu}}(n+1)}$ is special, then $\mathcal{D}_{\underline{\hat{\mu}}(n)}$ contains $P_{\infty_{+}}$at least twice.

Items $(i)$ and $($ ii $)$ hold if $n+1$ is replaced by $n-1$ and $P_{\infty_{+}}$by $P_{\infty_{-}}$.

Proof. (i) Suppose one point in $\mathcal{D}_{\hat{\mu}(n+1)}$ equals $P_{\infty_{-}}$and denote the remaining ones by $\mathcal{D}_{\underline{\underline{\mu}}(n+1)}$. Then (4.2) teills us

$$
\underline{\alpha}_{Q_{0}}\left(\mathcal{D}_{\underline{\tilde{\mu}}(n+1)}\right)+\underline{A}_{Q_{0}}\left(P_{\infty_{+}}\right)=\underline{\alpha}_{Q_{0}}\left(\mathcal{D}_{\underline{\hat{\mu}}(n)}\right) .
$$

Since we assumed $\mathcal{D}_{\underline{\hat{\mu}}(n)}$ to be nonspecial, we have

$$
\mathcal{D}_{\underline{\hat{\mu}}(n)}=\mathcal{D}_{\underline{\tilde{\mu}}(n+1)}+\mathcal{D}_{P_{\infty_{+}}}
$$

contradicting finiteness of $\mathcal{D}_{\underline{\hat{\mu}}(n)}$. 
(ii) We choose $Q_{0}$ to be a branch point of $\mathcal{K}_{p}$ such that $\underline{A}_{Q_{0}}\left(P^{*}\right)=-\underline{A}_{Q_{0}}(P)$. In particular, if $\mathcal{D}_{\hat{\mu}(n+1)}$ is special, then it contains a pair of points $\left(Q, Q^{*}\right)$ whose contribution will cancel under the Abel map, that is, $\underline{\alpha}_{Q_{0}}\left(\mathcal{D}_{\hat{\mu}(n+1)}\right)=$ $\underline{\alpha}_{Q_{0}}\left(\mathcal{D}_{\underline{\hat{\hat{\nu}}}(n+1)}\right)$ for some $\mathcal{D}_{\underline{\hat{\nu}}(n+1)} \in \operatorname{Sym}^{p-2}\left(\mathcal{K}_{p}\right)$. But invoking (4.2) shows that

$$
\underline{\alpha}_{Q_{0}}\left(\mathcal{D}_{\underline{\hat{\mu}}(n)}\right)=\underline{\alpha}_{Q_{0}}\left(\mathcal{D}_{\underline{\hat{\nu}}(n+1)}\right)+2 \underline{A}_{Q_{0}}\left(P_{\infty_{+}}\right) .
$$

As $\mathcal{D}_{\underline{\hat{\mu}}(n)}$ was assumed nonspecial, this shows that $\mathcal{D}_{\underline{\hat{\mu}}(n)}=\mathcal{D}_{\underline{\hat{\nu}}(n+1)}+2 \mathcal{D}_{P_{\infty_{+}}}$, as claìmed.

This yields the following behavior of $\mathcal{D}_{\hat{\mu}(n)}$ if we start with some nonspecial finite initial divisor $\mathcal{D}_{\hat{\mu}\left(n_{0}\right)}$ : As $n$ increases, $\mathcal{D}_{\hat{\mu}(n)}$ stays nonspecial as long as it remains finite. If it becomes infinite, then it is still nonspecial and contains $P_{\infty_{+}}$at least once (but not $P_{\infty_{-}}$). Further increasing $n$, all instances of $P_{\infty_{+}}$will be rendered into $P_{\infty_{-}}$step by step, until we have again a nonspecial divisor that has the same number of $P_{\infty_{-}}$as the first infinite one had $P_{\infty_{+}}$. Generically, we expect the subsequent divisor to be finite and nonspecial again.

Next we show that most initial divisors are nice in the sense that their iterates stay away from $P_{\infty_{ \pm}}$. Since we want to show that this set is of full measure, it will be convenient for us to identify $\operatorname{Sym}^{p}\left(\mathcal{K}_{p}\right)$ with the Jacobi variety $J\left(\mathcal{K}_{p}\right)$ via the Abel map and take the Haar measure on $J\left(\mathcal{K}_{p}\right)$. Of course, the Abel map is only injective when restricted to the set of nonspecial divisors, but these are the only ones we are interested in.

Lemma 4.2. The set $\mathcal{M}_{0} \subset \operatorname{Sym}^{p}\left(\mathcal{K}_{p}\right)$ of initial divisors $\mathcal{D}_{\hat{\mu}\left(n_{0}\right)}$ for which $\mathcal{D}_{\hat{\hat{\mu}}(n)}$, defined via (4.2), is finite and hence nonspecial for all $n \in \mathbb{Z}$, forms a dense set of full measure in the set $\operatorname{Sym}^{p}\left(\mathcal{K}_{p}\right)$ of nonnegative divisors of degree $p$.

Proof. Let $\mathcal{M}_{\infty}$ be the set of divisors in $\operatorname{Sym}^{p}\left(\mathcal{K}_{p}\right)$ for which (at least) one point is equal to $P_{\infty_{+}}$. The image $\underline{\alpha}_{Q_{0}}\left(\mathcal{M}_{\infty}\right)$ of $\mathcal{M}_{\infty}$ is given by

$$
\underline{\alpha}_{Q_{0}}\left(\mathcal{M}_{\infty}\right)=\underline{A}_{Q_{0}}\left(P_{\infty_{+}}\right)+\underline{\alpha}_{Q_{0}}\left(\operatorname{Sym}^{p-1}\left(\mathcal{K}_{p}\right)\right) \subset J\left(\mathcal{K}_{p}\right) .
$$

Since the (complex) dimension of $\operatorname{Sym}^{p-1}\left(\mathcal{K}_{p}\right)$ is $p-1$, its image must be of measure zero by Sard's theorem (see, e.g., [1, Sect. 3.6]). Similarly, let $\mathcal{M}_{\mathrm{sp}}$ be the set of special divisors, then its image is given by

$$
\underline{\alpha}_{Q_{0}}\left(\mathcal{M}_{\mathrm{sp}}\right)=\underline{\alpha}_{Q_{0}}\left(\operatorname{Sym}^{p-2}\left(\mathcal{K}_{p}\right)\right),
$$

assuming $Q_{0}$ to be a branch point. In particular, we conclude that

$$
\underline{\alpha}_{Q_{0}}\left(\mathcal{M}_{\mathrm{sp}}\right) \subset \underline{\alpha}_{Q_{0}}\left(\mathcal{M}_{\infty}\right)
$$


and thus $\underline{\alpha}_{Q_{0}}\left(\mathcal{M}_{\text {sing }}\right)=\underline{\alpha}_{Q_{0}}\left(\mathcal{M}_{\infty}\right)$ has measure zero, where

$$
\mathcal{M}_{\text {sing }}=\mathcal{M}_{\infty} \cup \mathcal{M}_{\text {sp }}
$$

Hence,

$$
\bigcup_{n \in \mathbb{Z}}\left(\underline{\alpha}_{Q_{0}}\left(\mathcal{M}_{\text {sing }}\right)+n \underline{A}_{P_{\infty_{-}}}\left(P_{\infty_{+}}\right)\right)
$$

is of measure zero as well. But this last set contains all initial divisors which will hit $P_{\infty_{+}}$or become special at some $n$. We denote by $\mathcal{M}_{0}$ the inverse image of the complement of the set (4.6) under the Abel map,

$$
\mathcal{M}_{0}=\underline{\alpha}_{Q_{0}}^{-1}\left(\operatorname{Sym}^{p}\left(\mathcal{K}_{p}\right) \backslash \bigcup_{n \in \mathbb{Z}}\left(\underline{\alpha}_{Q_{0}}\left(\mathcal{M}_{\text {sing }}\right)+n \underline{A}_{P_{\infty_{-}}}\left(P_{\infty_{+}}\right)\right)\right) .
$$

Since $\mathcal{M}_{0}$ is of full measure, it is automatically dense in $\operatorname{Sym}^{p}\left(\mathcal{K}_{p}\right)$.

We briefly illustrate some aspects of this analysis in the special case $p=1$ (i.e., the case where (3.1) represents an elliptic Riemann surface) in more detail.

Example 4.3. The case $p=1$.

In this case we have

$$
\begin{aligned}
F_{1}(z, n) & =z-\mu_{1}(n), \\
G_{2}(z, n) & =R_{4}\left(\hat{\mu}_{1}(n)\right)^{1 / 2}+(z-b(n)) F_{1}(z, n), \\
R_{4}(z) & =\prod_{m=0}^{3}\left(z-E_{m}\right),
\end{aligned}
$$

and hence a straightforward calculation shows that

$$
\begin{aligned}
G_{2}(z, n)^{2}-R_{4}(z) & =4 a(n)^{2}\left(z-\mu_{1}(n)\right)\left(z-\mu_{1}(n+1)\right) \\
& =\left(z-\mu_{1}(n)\right)\left(4 a(n)^{2} z-4 a(n)^{2} b(n)+\tilde{E}\right),
\end{aligned}
$$

where

$$
\tilde{E}=\frac{1}{8}\left(E_{0}+E_{1}-E_{2}-E_{3}\right)\left(E_{0}-E_{1}+E_{2}-E_{3}\right)\left(E_{0}-E_{1}-E_{2}+E_{3}\right) .
$$

Solving for $\mu_{1}(n+1)$, one obtains

$$
\mu_{1}(n+1)=b(n)-\frac{\tilde{E}}{4 a(n)^{2}} .
$$


This shows that $\mu_{1}\left(n_{0}+1\right) \rightarrow \infty$, in fact, $\mu_{1}\left(n_{0}+1\right)=O\left(a\left(n_{0}\right)^{-2}\right)$ as $a\left(n_{0}\right) \rightarrow 0$ during an appropriate deformation of the parameters $E_{m}, m=0, \ldots, 3$. In particular, as $a\left(n_{0}\right) \rightarrow 0$, one thus infers $b\left(n_{0}+1\right) \rightarrow \infty$ during such a deformation since

$$
b(n)=\frac{1}{2} \sum_{m=0}^{3} E_{m}-\mu_{1}(n), \quad n \in \mathbb{Z},
$$

specializing to $p=1$ in the trace formula (3.25). Next, we illustrate the set $\mathcal{M}_{\infty}$ in the case $p=1$. (We recall that $\mathcal{M}_{\text {sp }}=\emptyset$ and hence $\mathcal{M}_{\text {sing }}=\mathcal{M}_{\infty}$ if $p=1$.) By (4.2) one infers

$$
A_{P_{\infty_{+}}}\left(\hat{\mu}_{1}(n)\right)=A_{P_{\infty_{+}}}\left(\hat{\mu}_{1}\left(n_{0}\right)\right)+\left(n-n_{0}\right) A_{P_{\infty_{+}}}\left(P_{\infty_{-}}\right), \quad n, n_{0} \in \mathbb{Z} .
$$

We note that $\hat{\mu}_{1} \in \mathcal{M}_{\infty}$ is equivalent to

$$
\text { there is an } n \in \mathbb{Z} \text { such that } \hat{\mu}_{1}(n)=P_{\infty_{+}}\left(\text {or } P_{\infty_{-}}\right) \text {. }
$$

By (4.13), relation (4.14) is equivalent to

$$
A_{P_{\infty_{+}}}\left(\hat{\mu}_{1}\left(n_{0}\right)\right)+A_{P_{\infty_{+}}}\left(P_{\infty_{-}}\right) \mathbb{Z}=0 \quad\left(\bmod L_{1}\right) .
$$

Thus, $\mathcal{D}_{\hat{\mu}_{1}\left(n_{0}\right)} \in \mathcal{M}_{0} \subset \mathcal{K}_{1}$ if and only if

$$
A_{P_{\infty_{+}}}\left(\hat{\mu}_{1}\left(n_{0}\right)\right)+A_{P_{\infty_{+}}}\left(P_{\infty_{-}}\right) \mathbb{Z} \neq 0 \quad\left(\bmod L_{1}\right)
$$

or equivalently, if and only if

$$
A_{P_{\infty_{-}}}\left(\hat{\mu}_{1}\left(n_{0}\right)\right)+A_{P_{\infty_{-}}}\left(P_{\infty_{+}}\right) \mathbb{Z} \neq 0 \quad\left(\bmod L_{1}\right) .
$$

Next, we describe the stationary Toda algorithm. Since this is a somewhat lengthy affair, we will break it up into several steps.

\section{The stationary (complex) Toda algorithm:}

We prescribe the following data:

(i) The set

$$
\left\{E_{m}\right\}_{m=0}^{2 p+1} \subset \mathbb{C}, \quad E_{m} \neq E_{m^{\prime}} \text { for } m \neq m^{\prime}, m, m^{\prime}=0, \ldots, 2 p+1
$$

for some fixed $p \in \mathbb{N}$. Given $\left\{E_{m}\right\}_{m=0}^{2 p+1}$, we introduce the function $R_{2 p+2}$ and the (nonsingular) hyperelliptic curve $\mathcal{K}_{p}$ as in (3.1). 
(ii) The nonspecial divisor

$$
\mathcal{D}_{\underline{\hat{\mu}}\left(n_{0}\right)} \in \operatorname{Sym}^{p}\left(\mathcal{K}_{p}\right)
$$

where $\underline{\hat{\mu}}\left(n_{0}\right)$ is of the form

$$
\begin{aligned}
\underline{\hat{\mu}}\left(n_{0}\right) & =\left\{\hat{\mu}_{1}\left(n_{0}\right), \ldots, \hat{\mu}_{p}\left(n_{0}\right)\right\} \\
& =\{\underbrace{\hat{\mu}_{1}\left(n_{0}\right), \ldots, \hat{\mu}_{1}\left(n_{0}\right)}_{p_{1}\left(n_{0}\right) \text { times }}, \ldots, \underbrace{\hat{\mu}_{q\left(n_{0}\right)}, \ldots, \hat{\mu}_{q\left(n_{0}\right)}}_{p_{q\left(n_{0}\right)}\left(n_{0}\right) \text { times }}\}
\end{aligned}
$$

with

$$
\begin{aligned}
& \hat{\mu}_{k}\left(n_{0}\right)=\left(\mu_{k}\left(n_{0}\right), y\left(\hat{\mu}_{k}\left(n_{0}\right)\right)\right), \\
& \mu_{k}\left(n_{0}\right) \neq \mu_{k^{\prime}}\left(n_{0}\right) \text { for } k \neq k^{\prime}, k, k^{\prime}=1, \ldots, q\left(n_{0}\right),
\end{aligned}
$$

and

$$
p_{k}\left(n_{0}\right) \in \mathbb{N}, k=1, \ldots, q\left(n_{0}\right), \quad \sum_{k=1}^{q\left(n_{0}\right)} p_{k}\left(n_{0}\right)=p .
$$

With $\left\{E_{m}\right\}_{m=0}^{2 p+1}$ and $\mathcal{D}_{\underline{\hat{u}}\left(n_{0}\right)}$ prescribed, we next introduce the following quantities (for $z \in \mathbb{C}$ ):

$$
F_{p}\left(z, n_{0}\right)=\prod_{k=1}^{q\left(n_{0}\right)}\left(z-\mu_{k}\left(n_{0}\right)\right)^{p_{k}\left(n_{0}\right)}
$$

$$
\begin{gathered}
T_{p-1}\left(z, n_{0}\right)=-F_{p}\left(z, n_{0}\right) \sum_{k=1}^{q\left(n_{0}\right)} \sum_{\ell=0}^{p_{k}\left(n_{0}\right)-1} \frac{\left.\left(d^{\ell}\left(R_{2 p+2}(\zeta)^{1 / 2}\right) / d \zeta^{\ell}\right)\right|_{\zeta=\mu_{k}\left(n_{0}\right)}}{\ell !\left(p_{k}\left(n_{0}\right)-\ell-1\right) !} \\
\times\left.\left(\frac{d^{p_{k}\left(n_{0}\right)-\ell-1}}{d \zeta^{p_{k}\left(n_{0}\right)-\ell-1}}\left((z-\zeta)^{-1} \prod_{k^{\prime}=1, k^{\prime} \neq k}^{q\left(n_{0}\right)}\left(\zeta-\mu_{k^{\prime}}\left(n_{0}\right)\right)^{-p_{k^{\prime}}\left(n_{0}\right)}\right)\right)\right|_{\zeta=\mu_{k}\left(n_{0}\right)} \\
\quad b\left(n_{0}\right)=\frac{1}{2} \sum_{m=0}^{2 p+1} E_{m}-\sum_{k=1}^{q\left(n_{0}\right)} p_{k}\left(n_{0}\right) \mu_{k}\left(n_{0}\right) \\
G_{p+1}\left(z, n_{0}\right)=-\left(z-b\left(n_{0}\right)\right) F_{p}\left(z, n_{0}\right)+T_{p-1}\left(z, n_{0}\right)
\end{gathered}
$$

Here the sign of the square root in (4.24) is chosen according to (4.21),

$$
\begin{array}{r}
\hat{\mu}_{k}\left(n_{0}\right)=\left(\mu_{k}\left(n_{0}\right), y\left(\hat{\mu}_{k}\left(n_{0}\right)\right)\right)=\left(\mu_{k}\left(n_{0}\right), R_{2 p+2}\left(\mu_{k}\left(n_{0}\right)\right)^{1 / 2}\right), \\
k=1, \ldots, q\left(n_{0}\right) .
\end{array}
$$


Next we record a series of facts:

(I) By construction (cf. Lemma B.1),

$$
\begin{gathered}
T_{p-1}^{(\ell)}\left(\mu_{k}\left(n_{0}\right), n_{0}\right)=-\left.\frac{d^{\ell}\left(R_{2 p+2}(z)^{1 / 2}\right)}{d z^{\ell}}\right|_{z=\mu_{k}\left(n_{0}\right)}=G_{p+1}^{(\ell)}\left(\mu_{k}\left(n_{0}\right), n_{0}\right), \\
\ell=0, \ldots, p_{k}\left(n_{0}\right)-1, k=1, \ldots, q\left(n_{0}\right),
\end{gathered}
$$

(here the superscript $(\ell)$ denotes $\ell$ derivatives w.r.t. $z$ ) and hence

$$
\hat{\mu}_{k}\left(n_{0}\right)=\left(\mu_{k}\left(n_{0}\right),-G_{p+1}\left(\mu_{k}\left(n_{0}\right), n_{0}\right)\right), \quad k=1, \ldots, q\left(n_{0}\right) .
$$

(II) Since $\mathcal{D}_{\underline{\hat{\mu}}\left(n_{0}\right)}$ is nonspecial by hypothesis, one concludes that

$$
p_{k}\left(n_{0}\right) \geq 2 \text { implies } R_{2 p+2}\left(\mu_{k}\left(n_{0}\right)\right) \neq 0, \quad k=1, \ldots, q\left(n_{0}\right) .
$$

(III) By (I) and (II) one computes

$$
\begin{aligned}
& \left.\frac{d^{\ell}\left(G_{p+1}\left(z, n_{0}\right)^{2}\right)}{d z^{\ell}}\right|_{z=\mu_{k}\left(n_{0}\right)}=\left.\frac{d^{\ell} R_{2 p+2}(z)}{d z^{\ell}}\right|_{z=\mu_{k}\left(n_{0}\right)}, \\
& z \in \mathbb{C}, \quad \ell=0, \ldots, p_{k}\left(n_{0}\right)-1, k=1, \ldots, q\left(n_{0}\right) .
\end{aligned}
$$

(IV) By (4.26) and (4.31) one infers that $F_{p}$ divides $R_{2 p+2}-G_{p+1}^{2}$.

(V) By (4.25) and (4.26) one verifies that

$$
R_{2 p+2}(z)-G_{p+1}\left(z, n_{0}\right)^{2} \underset{z \rightarrow \infty}{=} O\left(z^{2 p}\right) .
$$

By (IV) and (4.32) we may write

$$
R_{2 p+2}(z)-G_{p+1}\left(z, n_{0}\right)^{2}=F_{p}\left(z, n_{0}\right) \check{F}_{p-r}\left(z, n_{0}+1\right), \quad z \in \mathbb{C},
$$

for some $r \in\{0, \ldots, p\}$, where the polynomial $\check{F}_{p-r}$ has degree $p-r$. If in fact $\check{F}_{0}=0$, then $R_{2 p+2}(z)=G_{p+1}\left(z, n_{0}\right)^{2}$ would yield double zeros of $R_{2 p+2}$, contradicting our basic hypothesis (4.18). Thus we conclude that in the case $r=p, \check{F}_{0}$ cannot vanish identically and hence we may break up (4.33) in the following manner

$$
\check{\phi}\left(P, n_{0}\right)=\frac{y-G_{p+1}\left(z, n_{0}\right)}{F_{p}\left(z, n_{0}\right)}=\frac{\check{F}_{p-r}\left(z, n_{0}+1\right)}{y+G_{p+1}\left(z, n_{0}\right)}, \quad P=(z, y) \in \mathcal{K}_{p} .
$$

Next we decompose

$$
\check{F}_{p-r}\left(z, n_{0}+1\right)=\check{C} \prod_{j=1}^{p-r}\left(z-\mu_{j}\left(n_{0}+1\right)\right), \quad z \in \mathbb{C},
$$


where $\check{C} \in \mathbb{C} \backslash\{0\}$ and $\left\{\mu_{j}\left(n_{0}+1\right)\right\}_{j=1}^{p-r} \subset \mathbb{C}$ (if $r=p$ we follow the usual convention and replace the product in (4.35) by 1). By inspection of the local zeros and poles as well as the behavior near $P_{\infty_{ \pm}}$of the function $\check{\phi}\left(\cdot, n_{0}\right)$, its divisor, $\left(\check{\phi}\left(\cdot, n_{0}\right)\right)$, is given by

$$
\left(\check{\phi}\left(\cdot, n_{0}\right)\right)=\mathcal{D}_{P_{\infty_{+}} \underline{\hat{\mu}}\left(n_{0}+1\right)}-\mathcal{D}_{P_{\infty_{-}} \underline{\hat{\mu}}\left(n_{0}\right)},
$$

where

$$
\underline{\hat{\mu}}\left(n_{0}+1\right)=\{\hat{\mu}_{1}\left(n_{0}+1\right), \ldots, \hat{\mu}_{p-r}\left(n_{0}+1\right), \underbrace{P_{\infty_{+}}, \ldots, P_{\infty_{+}}}_{r \text { times }}\} .
$$

In particular,

$$
\mathcal{D}_{\underline{\hat{\mu}}\left(n_{0}+1\right)} \text { is a finite divisor if and only if } r=0 .
$$

We note that

$$
\underline{\alpha}_{Q_{0}}\left(\mathcal{D}_{\underline{\hat{\mu}}\left(n_{0}+1\right)}\right)=\underline{\alpha}_{Q_{0}}\left(\mathcal{D}_{\underline{\hat{\mu}}\left(n_{0}\right)}\right)-\underline{A}_{P_{\infty_{-}}}\left(P_{\infty_{+}}\right),
$$

in accordance with (4.2).

(VI) Assuming that (4.32) is precisely of order $z^{2 p}$ as $z \rightarrow \infty$, that is, assuming $r=0$ in (4.33), we rewrite (4.33) in the more appropriate manner

$$
R_{2 p+2}(z)-G_{p+1}\left(z, n_{0}\right)^{2}=-4 a\left(n_{0}\right)^{2} F_{p}\left(z, n_{0}\right) F_{p}\left(z, n_{0}+1\right), \quad z \in \mathbb{C},
$$

where we introduced the coefficient $a\left(n_{0}\right)^{2}$ to make $F_{p}\left(\cdot, n_{0}+1\right)$ a monic polynomial of degree $p$. (We will later discuss conditions which indeed guarantee that $r=0$, cf. (4.38) and the discussion in step (XI) below.) By construction, $F_{p}\left(\cdot, n_{0}+1\right)$ is then of the type

$$
\begin{aligned}
F_{p}\left(z, n_{0}+1\right) & =\prod_{k=1}^{q\left(n_{0}+1\right)}\left(z-\mu_{k}\left(n_{0}+1\right)\right)^{p_{k}\left(n_{0}+1\right)}, \quad \sum_{k=1}^{q\left(n_{0}+1\right)} p_{k}\left(n_{0}+1\right)=p \\
\mu_{k}\left(n_{0}+1\right) & \neq \mu_{k^{\prime}}\left(n_{0}+1\right) \text { for } k \neq k^{\prime}, k, k^{\prime}=1, \ldots, q\left(n_{0}+1\right), \quad z \in \mathbb{C},
\end{aligned}
$$

and we define

$$
\hat{\mu}_{k}\left(n_{0}+1\right)=\left(\mu_{k}\left(n_{0}+1\right), G_{p+1}\left(\mu_{k}\left(n_{0}+1\right), n_{0}\right)\right), \quad k=1, \ldots, q\left(n_{0}+1\right) .
$$

Moreover, we introduce the divisor

$$
\mathcal{D}_{\underline{\hat{\mu}}\left(n_{0}+1\right)} \in \operatorname{Sym}^{p}\left(\mathcal{K}_{p}\right)
$$


by

$$
\begin{aligned}
\underline{\hat{\mu}}\left(n_{0}+1\right) & =\left\{\hat{\mu}_{1}\left(n_{0}+1\right), \ldots, \hat{\mu}_{p}\left(n_{0}+1\right)\right\} \\
& =\{\underbrace{\hat{\mu}_{1}\left(n_{0}+1\right), \ldots, \hat{\mu}_{1}\left(n_{0}+1\right)}_{p_{1}\left(n_{0}+1\right) \text { times }}, \ldots, \underbrace{\hat{\mu}_{q\left(n_{0}+1\right)}, \ldots, \hat{\mu}_{q\left(n_{0}+1\right)}}_{p_{q\left(n_{0}+1\right)}\left(n_{0}+1\right) \text { times }}\} .
\end{aligned}
$$

In particular, because of the definition $(4.42), \mathcal{D}_{\hat{\underline{\mu}}\left(n_{0}+1\right)}$ is nonspecial and hence

(4.45) $p_{k}\left(n_{0}+1\right) \geq 2$ implies $R_{2 p+2}\left(\mu_{k}\left(n_{0}+1\right)\right) \neq 0, \quad k=1, \ldots, q\left(n_{0}+1\right)$.

Again we note that

$$
\underline{\alpha}_{Q_{0}}\left(\mathcal{D}_{\underline{\hat{\mu}}\left(n_{0}+1\right)}\right)=\underline{\alpha}_{Q_{0}}\left(\mathcal{D}_{\underline{\hat{\mu}}\left(n_{0}\right)}\right)-\underline{A}_{P_{\infty_{-}}}\left(P_{\infty_{+}}\right),
$$

in accordance with (4.2).

(VII) Introducing

$$
b\left(n_{0}+1\right)=\frac{1}{2} \sum_{m=0}^{2 p+1} E_{m}-\sum_{k=1}^{q\left(n_{0}+1\right)} p_{k}\left(n_{0}+1\right) \mu_{k}\left(n_{0}+1\right),
$$

and interpolating $G_{p+1}\left(\cdot, n_{0}\right)$ with $F_{p}\left(\cdot, n_{0}+1\right)$ rather than $F_{p}\left(\cdot, n_{0}\right)$ yields

$$
G_{p+1}\left(z, n_{0}\right)=-\left(z-b\left(n_{0}+1\right)\right) F_{p}\left(z, n_{0}+1\right)-T_{p-1}\left(z, n_{0}+1\right), \quad z \in \mathbb{C},
$$

where

$$
\begin{aligned}
& T_{p-1}\left(z, n_{0}+1\right)=F_{p}\left(z, n_{0}+1\right) \\
& \times \sum_{k=1}^{q\left(n_{0}+1\right)} \sum_{\ell=0}^{p_{k}\left(n_{0}+1\right)-1} \frac{\left.\left(d^{\ell}\left(R_{2 p+2}(\zeta)^{1 / 2}\right) / d \zeta^{\ell}\right)\right|_{\zeta=\mu_{k}\left(n_{0}+1\right)}}{\ell !\left(p_{k}\left(n_{0}+1\right)-\ell-1\right) !} \\
& \times\left(\frac { d ^ { p _ { k } ( n _ { 0 } + 1 ) - \ell - 1 } } { d \zeta ^ { p _ { k } ( n _ { 0 } + 1 ) - \ell - 1 } } \left((z-\zeta)^{-1}\right.\right. \\
& \left.\left.\times \prod_{k^{\prime}=1, k^{\prime} \neq k}^{q\left(n_{0}+1\right)}\left(\zeta-\mu_{k^{\prime}}\left(n_{0}+1\right)\right)^{-p_{k^{\prime}}\left(n_{0}+1\right)}\right)\right)\left.\right|_{\zeta=\mu_{k}\left(n_{0}+1\right)}
\end{aligned}
$$

Here the sign of the square root in (4.49) is chosen in accordance with (4.42), that is,

$$
\begin{array}{r}
\hat{\mu}_{k}\left(n_{0}+1\right)=\left(\mu_{k}\left(n_{0}+1\right), y\left(\hat{\mu}_{k}\left(n_{0}+1\right)\right)\right) \\
=\left(\mu_{k}\left(n_{0}+1\right), G_{p+1}\left(\mu_{k}\left(n_{0}+1\right), n_{0}\right)\right) \\
=\left(\mu_{k}\left(n_{0}+1\right), R_{2 p+2}\left(\mu_{k}\left(n_{0}+1\right)\right)^{1 / 2}\right), \\
\quad k=1, \ldots, q\left(n_{0}+1\right) .
\end{array}
$$


(VIII) An explicit computation of $a\left(n_{0}\right)^{2}$ then yields

$$
\begin{aligned}
a\left(n_{0}\right)^{2}= & \frac{1}{2} \sum_{k=1}^{q\left(n_{0}\right)} \frac{\left.\left(d^{\ell}\left(R_{2 p+2}(z)^{1 / 2}\right) / d z^{\ell}\right)\right|_{z=\mu_{k}\left(n_{0}\right)}}{\left(p_{k}\left(n_{0}\right)-1\right) !} \\
& \times \prod_{k^{\prime}=1, k^{\prime} \neq k}^{q\left(n_{0}\right)}\left(\mu_{k}\left(n_{0}\right)-\mu_{k^{\prime}}\left(n_{0}\right)\right)^{-p_{k}\left(n_{0}\right)}+\frac{1}{4}\left(b^{(2)}\left(n_{0}\right)-b\left(n_{0}\right)^{2}\right) .
\end{aligned}
$$

Here and in the following we abbreviate

$$
b^{(2)}(n)=\frac{1}{2} \sum_{m=0}^{2 p+1} E_{m}^{2}-\sum_{k=1}^{q(n)} p_{k}(n) \mu_{k}(n)^{2}
$$

for an appropriate range of $n \in \mathbb{N}$.

The result (4.51) is obtained as follows: One starts from the identity (4.40), inserts the expressions (4.23) and (4.26) for $F_{p}\left(\cdot, n_{0}\right)$ and $G_{p+1}\left(\cdot, n_{0}\right)$, respectively, then inserts the explicit form $(4.24)$ of $T_{p-1}\left(\cdot, n_{0}\right)$, and finally collects all terms of order $z^{2 p}$ as $z \rightarrow \infty$. An entirely elementary but fairly tedious calculation then produces (4.51).

In the special case $q\left(n_{0}\right)=p, p_{k}\left(n_{0}\right)=1, k=1, \ldots, p,(4.51)$ and (4.52) reduce to (3.26) and (3.24) (for $k=2$ ).

(IX) Introducing

$$
G_{p+1}\left(z, n_{0}+1\right)=-\left(z-b\left(n_{0}+1\right)\right) F_{p}\left(z, n_{0}+1\right)+T_{p-1}\left(z, n_{0}+1\right)
$$

one then obtains

$$
G_{p+1}\left(z, n_{0}+1\right)=-G_{p+1}\left(z, n_{0}\right)-2\left(z-b\left(n_{0}+1\right)\right) F_{p}\left(z, n_{0}+1\right) .
$$

(X) At this point one can iterate the procedure step by step to construct $F_{p}(\cdot, n), G_{p+1}(\cdot, n), T_{p-1}(\cdot, n), a(n), b(n), \mu_{k}(n), k=1, \ldots, q(n)$, etc., for $n \in\left[n_{0}, \infty\right) \cap \mathbb{Z}$, subject to the following assumption (cf. (4.38)) at each step:

$$
\mathcal{D}_{\underline{\hat{\mu}}(n+1)} \text { is a finite divisor (and hence } a(n) \neq 0 \text { ) }
$$

$$
\text { for all } n \in\left[n_{0}, \infty\right) \cap \mathbb{Z} \text {. }
$$

The formalism is symmetric with respect to $n_{0}$ and can equally well be developed for $n \in\left(-\infty, n_{0}\right] \cap \mathbb{Z}$ subject to the analogous assumption

$$
\begin{array}{r}
\left.\mathcal{D}_{\underline{\hat{\mu}}(n-1)} \text { is a finite divisor (and hence } a(n) \neq 0\right) \\
\text { for all } n \in\left(-\infty, n_{0}\right] \cap \mathbb{Z} .
\end{array}
$$


Indeed, one first interpolates $G_{p+1}\left(\cdot, n_{0}-1\right)$ with the help of $F_{p}\left(\cdot, n_{0}\right)$, then with $F_{p}\left(\cdot, n_{0}-1\right)$, etc.

Moreover, we once again remark for consistency reasons that

$$
\underline{\alpha}_{Q_{0}}\left(\mathcal{D}_{\underline{\hat{\mu}}(n)}\right)=\underline{\alpha}_{Q_{0}}\left(\mathcal{D}_{\underline{\hat{\mu}}\left(n_{0}\right)}\right)-\left(n-n_{0}\right) \underline{A}_{P_{\infty_{-}}}\left(P_{\infty_{+}}\right), \quad n \in \mathbb{Z},
$$

in agreement with our starting point (4.2).

(XI) Choosing the initial data $\mathcal{D}_{\underline{\hat{\mu}}\left(n_{0}\right)}$ such that

$$
\mathcal{D}_{\underline{\hat{\mu}}\left(n_{0}\right)} \in \mathcal{M}_{0},
$$

where $\mathcal{M}_{0} \subset \operatorname{Sym}^{p}\left(\mathcal{K}_{p}\right)$ is the set of finite divisors introduced in Lemma 4.2, then guarantees that assumptions (4.55) and (4.56) are satisfied for all $n \in \mathbb{Z}$.

(XII) Performing these iterations for all $n \in \mathbb{Z}$, one then arrives at the following set of equations for $F_{p}$ and $G_{p+1}$ after the following elementary manipulations: Utilizing

$$
G_{p+1}^{2}-4 a^{2} F_{p} F_{p}^{+}=R_{2 p+2}=\left(G_{p+1}^{-}\right)^{2}-4\left(a^{-}\right)^{2} F_{p}^{-} F_{p},
$$

and inserting

$$
G_{p+1}^{+}=-G_{p+1}-2\left(z-b^{+}\right) F_{p}^{+}
$$

into

$$
G_{p+1}^{2}-\left(G_{p+1}^{-}\right)^{2}-4 a^{2} F_{p} F_{p}^{+}+4\left(a^{-}\right)^{2} F_{p}^{-} F_{p}=0
$$

then yields

$$
2 a^{2} F_{p}^{+}-2\left(a^{-}\right)^{2} F_{p}^{-}+(z-b)\left(G_{p+1}-G_{p+1}^{-}\right)=0 .
$$

Subtracting (4.60) from its shifted version $G_{p+1}=-G_{p+1}^{-}-2(z-b) F_{p}$ then also yields

$$
2\left(z-b^{+}\right) F_{p}^{+}-2(z-b) F_{p}+G_{p+1}^{+}-G_{p+1}^{-}=0 .
$$

As discussed in Section 2, (4.62) and (4.63) are equivalent to the stationary Lax and zero-curvature equations (2.15) and (2.60). At this stage we have verified the basic hypotheses of Section 3 (i.e., (3.2) and the assumption that $a, b$ satisfy the $p$ th stationary Toda system (2.20)) and hence all results of Section 3 apply.

Finally, we briefly summarize these considerations: 
Theorem 4.4. Suppose the set $\left\{E_{m}\right\}_{m=0}^{2 p+1} \subset \mathbb{C}$ satisfies $E_{m} \neq E_{m^{\prime}}$ for $m \neq m^{\prime}, m, m^{\prime}=0, \ldots, 2 p+1$, and introduce the function $R_{2 p+2}$ and the hyperelliptic curve $\mathcal{K}_{p}$ as in (3.1). Choose a nonspecial divisor $\mathcal{D}_{\underline{\hat{\mu}}\left(n_{0}\right)} \in \mathcal{M}_{0}$, where $\mathcal{M}_{0} \subset \operatorname{Sym}^{p}\left(\mathcal{K}_{p}\right)$ is the set of finite divisors introduced in Lemma 4.2. Then the stationary (complex) Toda algorithm as outlined in steps (I)-(XII) produces solutions $a, b$ of the pth stationary Toda system,

$$
\mathrm{s}-\mathrm{Tl}_{p}(a, b)=\left(\begin{array}{c}
f_{p+1}^{+}-f_{p+1} \\
g_{p+1}-g_{p+1}^{-}
\end{array}\right)=0, \quad p \in \mathbb{N}_{0}
$$

satisfying (3.2) and

$$
\begin{aligned}
a(n)^{2}= & \frac{1}{2} \sum_{k=1}^{q(n)} \frac{\left.\left(d^{\ell}\left(R_{2 p+2}(z)^{1 / 2}\right) / d z^{\ell}\right)\right|_{z=\mu_{k}(n)}}{\left(p_{k}(n)-1\right) !} \\
& \times \prod_{k^{\prime}=1, k^{\prime} \neq k}^{q(n)}\left(\mu_{k}(n)-\mu_{k^{\prime}}(n)\right)^{-p_{k}(n)}+\frac{1}{4}\left(b^{(2)}(n)-b(n)^{2}\right), \\
b(n)= & \frac{1}{2} \sum_{m=0}^{2 p+1} E_{m}-\sum_{k=1}^{q(n)} p_{k}(n) \mu_{k}(n), \quad n \in \mathbb{Z} .
\end{aligned}
$$

Moreover, Lemmas 3.2-3.4 apply.

Remark 4.5. Suppose that the hypotheses of the previous theorem are satisfied and that $a\left(n_{0}\right), b\left(n_{0}\right), b\left(n_{0}+1\right), F_{p}\left(z, n_{0}\right), F_{p}\left(z, n_{0}+1\right), G_{p+1}\left(z, n_{0}\right)$, and $G_{p+1}\left(z, n_{0}+1\right)$ have already been computed using steps $(\mathbf{I})-(\mathbf{I X})$. Then, alternatively, one can use

$$
\begin{aligned}
\left(a^{-}\right)^{2} F_{p}^{-}= & a^{2} F_{p}^{+}+2^{-1}(z-b)\left(G_{p+1}-G_{p+1}^{+}\right)+(z-b)^{2} F_{p} \\
& -\left(z-b^{+}\right)(z-b) F_{p}^{+} \\
G_{p+1}^{-}= & 2\left(\left(z-b^{+}\right) F_{p}^{+}-(z-b) F_{p}\right)+G_{p+1}^{+}
\end{aligned}
$$

(derived from (2.60)) to compute $a(n), b(n), F_{p}(z, n), G_{p+1}(z, n)$ for $n<n_{0}$ and

$$
\begin{aligned}
a^{+} F_{p}^{++} & =a F_{p}-2^{-1}(z-b)\left(G_{p+1}^{+}-G_{p+1}\right), \\
G_{p+1}^{++} & =G_{p+1}-2\left(\left(z-b^{++}\right) F_{p}^{++}-\left(z-b^{+}\right) F_{p}^{+}\right)
\end{aligned}
$$

to compute $a(n-1), b(n), F_{p}(z, n), G_{p+1}(z, n)$ for $n>n_{0}+1$.

Theta function representations of $a$ and $b$ can now be derived in complete analogy to the self-adjoint case. Since the final results are formally the same 
as in the self-adjoint case we just refer, for instance, to [6], [7], [9], [10], [14, Sect. 1.3], [18], [19], [20], [21] (cf. also the appendix written in [8]), [25], [30, Appendix, Sect. 9], [32, Sect. 9.2], [33, Sect. 4.5].

The stationary (complex) Toda algorithm as outlined in steps (I )-(XII ), starting from a nonspecial divisor $\mathcal{D}_{\underline{\hat{\mu}}\left(n_{0}\right)} \in \mathcal{M}_{0}$, represents a solution of the inverse algebro-geometric spectral problem for generally non-self-adjoint Jacobi operators. While we do not assume periodicity (or even quasiperiodicity), let alone real-valuedness of the coefficients of the underlying Jacobi operator, once can view this algorithm as a continuation of the inverse periodic spectral problem started around 1975 (in the self-adjoint context) by Kac and van Moerbeke [15], [16] and Flaschka [12], continued in the seminal papers by van Moerbeke [24], Date and Tanaka [7], and Dubrovin, Matveev, and Novikov [10], and further developed by Krichever [18], McKean [23], van Moerbeke and Mumford [25], Mumford [26], and others, in part in the more general quasi-periodic algebro-geometric case.

We note that in general (i.e., unless one is, e.g., in the special periodic or self-adjoint case), $\mathcal{D}_{\hat{\mu}(n)}$ will get arbitrarily close to $P_{\infty_{+}}$since straight motions on the torus are generically dense (see e.g. [2, Sect. 51] or [17, Sects. $1.4,1.5])$. Thus, no uniform bound on the sequences $a(n), b(n)$ exists as $n$ varies in $\mathbb{Z}$. In particular, these complex-valued algebro-geometric solutions of some of the equations of the stationary Toda hierarchy, generally, will not be quasi-periodic (cf. the usual definition of quasi-periodic functions, e.g., in $[31$, p. 31]) with respect to $n$. For the special case of complex-valued and quasi-periodic Jacobi matrices where all quasi-periods are real-valued, we refer to [4] (cf. also [3]).

\section{Properties of algebro-geometric solutions of the time- dependent Toda hierarchy}

In this section we present a quick review of properties of algebro-geometric solutions of the time-dependent Toda hierarchy. Since this material is standard we omit all proofs and just refer to [6] (cf. also [14, Sect. 1.4], [32, Chs. 12, 13]) for detailed presentations and an extensive list references to the literature.

For most of this section we will make the following assumption:

Hypothesis 5.1. Suppose that $a, b$ satisfy

$$
\begin{aligned}
& a(\cdot, t), b(\cdot, t) \in \mathbb{C}^{\mathbb{Z}}, t \in \mathbb{R}, \quad a(n, \cdot), b(n, \cdot) \in C^{1}(\mathbb{R}), n \in \mathbb{Z}, \\
& a(n, t) \neq 0,(n, t) \in \mathbb{Z} \times \mathbb{R}
\end{aligned}
$$

and assume that the hyperelliptic curve $\mathcal{K}_{p}, p \in \mathbb{N}_{0}$, is nonsingular. 
In order to briefly analyze algebro-geometric solutions of the time-dependent Toda hierarchy we proceed as follows. Given $p \in \mathbb{N}_{0}$, consider a complexvalued solution $a^{(0)}, b^{(0)}$ of the $p$ th stationary Toda system s- $\operatorname{Tl}_{p}(a, b)=0$, associated with $\mathcal{K}_{p}$ and a given set of summation constants $\left\{c_{\ell}\right\}_{\ell=1, \ldots, p} \subset \mathbb{C}$. Next, let $r \in \mathbb{N}_{0}$; we intend to consider solutions $a=a\left(t_{r}\right), b=b\left(t_{r}\right)$ of the $r$ th $\mathrm{Tl}$ flow $\mathrm{Tl}_{r}(a, b)=0$ with $a\left(t_{0, r}\right)=a^{(0)}, b\left(t_{0, r}\right)=b^{(0)}$ for some $t_{0, r} \in \mathbb{R}$. To emphasize that the summation constants in the definitions of the stationary and the time-dependent $\mathrm{Tl}$ equations are independent of each other, we indicate this by adding a tilde on all the time-dependent quantities. Hence we shall employ the notation $\widetilde{P}_{2 r+2}, \widetilde{V}_{r+1}, \widetilde{F}_{r}, \widetilde{G}_{r+1}, \tilde{f}_{s}$, $\tilde{g}_{s}, \tilde{c}_{s}$, in order to distinguish them from $P_{2 p+2}, V_{p+1}, F_{p}, G_{p+1}, f_{\ell}, g_{\ell}, c_{\ell}$, in the following. In addition, we will follow a more elaborate notation inspired by Hirota's $\tau$-function approach and indicate the individual $r$ th $\mathrm{Tl}$ flow by a separate time variable $t_{r} \in \mathbb{R}$. More precisely, we will review properties of solutions $a, b$ of the time-dependent algebro-geometric initial value problem

$$
\begin{aligned}
& \widetilde{\mathrm{T}} l_{r}(a, b)=\left(\begin{array}{c}
a_{t_{r}}-a\left(\tilde{f}_{p+1}^{+}(a, b)-\tilde{f}_{p+1}(a, b)\right) \\
b_{t_{r}}+\tilde{g}_{p+1}(a, b)-\tilde{g}_{p+1}^{-}(a, b)
\end{array}\right)=0, \\
& \left.(a, b)\right|_{t_{r}=t_{0, r}}=\left(a^{(0)}, b^{(0)}\right), \\
& \mathrm{s}-\mathrm{Tl}_{p}\left(a^{(0)}, b^{(0)}\right)=\left(\begin{array}{c}
-a\left(f_{p+1}^{+}\left(p^{(0)}, q^{(0)}\right)-f_{p+1}\left(p^{(0)}, q^{(0)}\right)\right) \\
g_{p+1}\left(a^{(0)}, b^{(0)}\right)-g_{p+1}^{-}\left(a^{(0)}, b^{(0)}\right)
\end{array}\right)=0
\end{aligned}
$$

for some $t_{0, r} \in \mathbb{R}, p, r \in \mathbb{N}_{0}$, where $a=a\left(n, t_{r}\right), b=b\left(n, t_{r}\right)$ satisfy (5.1) and a fixed curve $\mathcal{K}_{p}$ is associated with the stationary solutions $a^{(0)}, b^{(0)}$ in (5.3). In terms of Lax pairs this amounts to solving

$$
\begin{aligned}
\frac{d}{d t_{r}} L\left(t_{r}\right)-\left[\widetilde{P}_{2 r+2}\left(t_{r}\right), L\left(t_{r}\right)\right] & =0, \quad t_{r} \in \mathbb{R}, \\
{\left[P_{2 p+2}\left(t_{0, r}\right), L\left(t_{0, r}\right)\right] } & =0 .
\end{aligned}
$$

Anticipating that the $\mathrm{Tl}$ flows are isospectral deformations of $L\left(t_{0, r}\right)$, we are going a step further replacing (5.5) by

$$
\left[P_{2 p+2}\left(t_{r}\right), L\left(t_{r}\right)\right]=0, \quad t_{r} \in \mathbb{R} .
$$

This then implies

$$
P_{2 p+2}\left(t_{r}\right)^{2}=R_{2 p+2}\left(L\left(t_{r}\right)\right)=\prod_{m=0}^{2 p+1}\left(L\left(t_{r}\right)-E_{m}\right), \quad t_{r} \in \mathbb{R} .
$$

Actually, instead of working with (5.4), (5.5), and (5.6), one can equivalently take the zero-curvature equations (2.63) as one's point of departure, that is, 
one can also start from

$$
\begin{aligned}
U_{t_{r}}+U \widetilde{V}_{r+1}-\widetilde{V}_{r+1}^{+} U & =0, \\
U V_{p+1}-V_{p+1}^{+} U & =0,
\end{aligned}
$$

where (cf. (2.23), (2.24), (2.58), (2.59))

$$
\begin{aligned}
U(z) & =\left(\begin{array}{cc}
0 & 1 \\
-a^{-} / a & (z-b) / a
\end{array}\right), \\
V_{p+1}(z) & =\left(\begin{array}{cc}
G_{p+1}^{-}(z) & 2 a^{-} F_{p}^{-}(z) \\
-2 a^{-} F_{p}(z) & 2(z-b) F_{p}+G_{p+1}(z)
\end{array}\right), \\
\widetilde{V}_{r+1}(z) & =\left(\begin{array}{cc}
\widetilde{G}_{r+1}^{-}(z) & 2 a^{-} \widetilde{F}_{r}^{-}(z) \\
-2 a^{-} \widetilde{F}_{r}(z) & 2(z-b) \widetilde{F}_{r}(z)+\widetilde{G}_{r+1}(z)
\end{array}\right),
\end{aligned}
$$

and

$$
\begin{aligned}
F_{p}(z) & =\sum_{\ell=0}^{p} f_{p-\ell} z^{\ell}=\prod_{j=1}^{p}\left(z-\mu_{j}\right), \quad f_{0}=1, \\
G_{p+1}(z) & =-z^{p+1}+\sum_{\ell=0}^{p} g_{p-\ell} z^{\ell}+f_{p+1}, \quad g_{0}=-c_{1}, \\
\widetilde{F}_{r}(z) & =\sum_{s=0}^{r} \tilde{f}_{r-s} z^{s}, \quad \tilde{f}_{0}=1, \\
\widetilde{G}_{r+1}(z) & =-z^{r+1}+\sum_{s=0}^{r} \tilde{g}_{r-s} z^{s}+\tilde{f}_{r+1}, \quad \tilde{g}_{0}=-\tilde{c}_{1},
\end{aligned}
$$

for fixed $p, r \in \mathbb{N}_{0}$. Here $f_{\ell}, \tilde{f}_{s}, g_{\ell}$, and $\tilde{g}_{s}, \ell=0, \ldots, p, s=0, \ldots, r$, are defined as in (2.4)-(2.6) with appropriate sets of summation constants $c_{\ell}$, $\ell \in \mathbb{N}$, and $\tilde{c}_{k}, k \in \mathbb{N}$. Explicitly, (5.8) and (5.9) are equivalent to (cf. (2.55), (2.56), (2.32), (2.33))

$$
\begin{aligned}
a_{t_{r}} & =-a\left(2\left(z-b^{+}\right) \widetilde{F}_{r}^{+}+\widetilde{G}_{r+1}^{+}+\widetilde{G}_{r+1}\right), \\
b_{t_{r}} & =2\left((z-b)^{2} \widetilde{F}_{r}+(z-b) \widetilde{G}_{r+1}+a^{2} \widetilde{F}_{r}^{+}-\left(a^{-}\right)^{2} \widetilde{F}_{r}^{-}\right), \\
0 & =2\left(z-b^{+}\right) F_{p}^{+}+G_{p+1}^{+}+G_{p+1}, \\
0 & =(z-b)^{2} F_{p}+(z-b) G_{p+1}+a^{2} F_{p}^{+}-\left(a^{-}\right)^{2} F_{p}^{-},
\end{aligned}
$$

respectively. In particular, (2.34) holds in the present $t_{r}$-dependent setting, that is,

$$
G_{p+1}^{2}-4 a^{2} F_{p} F_{p}^{+}=R_{2 p+2} .
$$


As in (3.6) one introduces

$$
\begin{array}{r}
\hat{\mu}_{j}\left(n, t_{r}\right)=\left(\mu_{j}\left(n, t_{r}\right),-G_{p+1}\left(\mu_{j}\left(n, t_{r}\right), n, t_{r}\right)\right) \in \mathcal{K}_{p}, \\
j=1, \ldots, p,\left(n, t_{r}\right) \in \mathbb{Z} \times \mathbb{R}, \\
\hat{\mu}_{j}^{+}\left(n, t_{r}\right)=\left(\mu_{j}^{+}\left(n, t_{r}\right), G_{p+1}\left(\mu_{j}^{+}\left(n, t_{r}\right), n, t_{r}\right)\right) \in \mathcal{K}_{p}, \\
j=1, \ldots, p,\left(n, t_{r}\right) \in \mathbb{Z} \times \mathbb{R},
\end{array}
$$

and notes that the regularity assumptions (5.1) on $a, b$ imply continuity of $\mu_{j}$ with respect to $t_{r} \in \mathbb{R}$ (away from collisions of zeros, $\mu_{j}$ are of course $C^{\infty}$ ).

In analogy to $(3.7),(3.8)$, one defines the meromorphic function $\phi\left(\cdot, n, t_{r}\right)$ on $\mathcal{K}_{p}$,

$$
\begin{aligned}
& \phi\left(P, n, t_{r}\right)=\frac{y-G_{p+1}\left(z, n, t_{r}\right)}{2 a\left(n, t_{r}\right) F_{p}\left(z, n, t_{r}\right)} \\
&=\frac{-2 a\left(n, t_{r}\right) F_{p}\left(z, n+1, t_{r}\right)}{y+G_{p+1}\left(z, n, t_{r}\right)}, \\
& P(z, y) \in \mathcal{K}_{p},\left(n, t_{r}\right) \in \mathbb{Z} \times \mathbb{R},
\end{aligned}
$$

with divisor $\left(\phi\left(\cdot, n, t_{r}\right)\right)$ of $\phi\left(\cdot, n, t_{r}\right)$ given by

$$
\left(\phi\left(\cdot, n, t_{r}\right)\right)=\mathcal{D}_{P_{\infty+\underline{\hat{\mu}}}\left(n+1, t_{r}\right)}-\mathcal{D}_{P_{\infty} \underline{\hat{\mu}}\left(n, t_{r}\right)},
$$

using (5.11) and (5.20).

The time-dependent Baker-Akhiezer function $\psi\left(P, n, n_{0}, t_{r}, t_{0, r}\right)$, meromorphic on $\mathcal{K}_{p} \backslash\left\{P_{\infty_{+}}, P_{\infty_{-}}\right\}$, is then defined in terms of $\phi$ by

$$
\begin{aligned}
& \psi\left(P, n, n_{0}, t_{r}, t_{0, r}\right) \\
& \quad=\exp \left(\int_{t_{0, r}}^{t_{r}} d s\left(2 a\left(n_{0}, s\right) \widetilde{F}_{r}\left(z, n_{0}, s\right) \phi\left(P, n_{0}, s\right)+\widetilde{G}_{r+1}\left(z, n_{0}, s\right)\right)\right)
\end{aligned}
$$

$$
\begin{array}{r}
\times \begin{cases}\prod_{m=n_{0}}^{n-1} \phi\left(P, m, t_{r}\right) & \text { for } n \geq n_{0}+1, \\
1 & \text { for } n=n_{0}, \\
\prod_{m=n}^{n_{0}-1} \phi\left(P, m, t_{r}\right)^{-1} & \text { for } n \leq n_{0}-1,\end{cases} \\
P \in \mathcal{K}_{p} \backslash\left\{P_{\infty_{ \pm}}\right\},\left(n, n_{0}, t_{r}, t_{0, r}\right) \in \mathbb{Z}^{2} \times \mathbb{R}^{2} .
\end{array}
$$

For subsequent purposes we also introduce the following Baker-Akhiezer vector,

$$
\begin{gathered}
\Psi\left(P, n, n_{0}, t_{r}, t_{0, r}\right)=\left(\begin{array}{c}
\psi^{-}\left(P, n, n_{0}, t_{r}, t_{0, r}\right) \\
\psi\left(P, n, n_{0}, t_{r}, t_{0, r}\right)
\end{array}\right), \\
P \in \mathcal{K}_{p} \backslash\left\{P_{\infty_{ \pm}}\right\},\left(n, n_{0}, t_{r}, t_{0, r}\right) \in \mathbb{Z}^{2} \times \mathbb{R}^{2} .
\end{gathered}
$$


Basic properties of $\phi, \psi$, and $\Psi$ are summarized in the following lemma.

Lemma 5.2. Assume Hypothesis 5.1 and suppose that $a, b$ satisfy (5.15)(5.18). In addition, let $P=(z, y) \in \mathcal{K}_{p} \backslash\left\{P_{\infty_{ \pm}}\right\},\left(n, n_{0}, t_{r}, t_{0, r}\right) \in \mathbb{Z}^{2} \times \mathbb{R}^{2}$, and $r \in \mathbb{N}_{0}$. Then $\phi$ satisfies

$$
\begin{aligned}
& a \phi(P)+a^{-}\left(\phi^{-}(P)\right)^{-1}=z-b, \\
& \phi_{t_{r}}(P)=-2 a\left(\widetilde{F}_{r}(z) \phi(P)^{2}+\widetilde{F}_{r}^{+}(z)\right)+2\left(z-b^{+}\right) \widetilde{F}_{r}^{+}(z) \phi(P) \\
& +\left(\widetilde{G}_{r+1}^{+}(z)-\widetilde{G}_{r+1}(z)\right) \phi(P), \\
& \phi(P) \phi\left(P^{*}\right)=\frac{F_{p}^{+}(z)}{F_{p}(z)}, \\
& \phi(P)-\phi\left(P^{*}\right)=\frac{y(P)}{a F_{p}(z)}, \\
& \phi(P)+\phi\left(P^{*}\right)=-\frac{G_{p+1}(z)}{a F_{p}(z)} .
\end{aligned}
$$

Moreover, $\psi$ and $\Psi$ satisfy

$$
\begin{aligned}
& (L-z(P)) \psi(P)=0, \quad\left(P_{2 p+2}-y(P)\right) \psi(P)=0 \\
& \psi_{t_{r}}(P)=\widetilde{P}_{2 r+2} \psi(P)
\end{aligned}
$$$$
=2 a \widetilde{F}_{r}(z) \psi^{+}(P)+\widetilde{G}_{r+1}(z) \psi(P),
$$

$$
\Psi^{+}(P)=U(z) \Psi(P), \quad y \Psi(P)=V_{p+1} \Psi(P),
$$

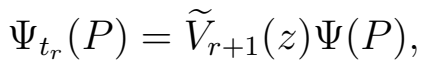

$$
\begin{aligned}
& \psi\left(P, n, n_{0}, t_{r}, t_{0, r}\right) \psi\left(P^{*}, n, n_{0}, t_{r}, t_{0, r}\right)=\frac{F_{p}\left(z, n, t_{r}\right)}{F_{p}\left(z, n_{0}, t_{0, r}\right)} \\
& a\left(n, t_{r}\right)\left(\psi\left(P, n, n_{0}, t_{r}, t_{0, r}\right) \psi\left(P^{*}, n+1, n_{0}, t_{r}, t_{0, r}\right)\right.
\end{aligned}
$$$$
\left.+\psi\left(P^{*}, n, n_{0}, t_{r}, t_{0, r}\right) \psi\left(P, n+1, n_{0}, t_{r}, t_{0, r}\right)\right)=-\frac{G_{p+1}\left(z, n, t_{r}\right)}{F_{p}\left(z, n_{0}, t_{0, r}\right)},
$$

$$
W\left(\psi\left(P, \cdot, n_{0}, t_{r}, t_{0, r}\right), \psi\left(P^{*}, \cdot, n_{0}, t_{r}, t_{0, r}\right)\right)=-\frac{y(P)}{F_{p}\left(z, n_{0}, t_{0, r}\right)} .
$$

In complete analogy to the case of stationary trace formulas one obtains trace formulas in the time-dependent setting (cf. the abbreviation (3.24)).

Lemma 5.3. Assume Hypothesis 5.1 and suppose that a,b satisfy (5.15) and (5.16). Then,

$$
b=\frac{1}{2} \sum_{m=0}^{2 p+1} E_{m}-\sum_{j=1}^{p} \mu_{j} .
$$


In addition, if for all $\left(n, t_{r}\right) \in \mathbb{Z} \times \mathbb{R}, \mu_{j}\left(n, t_{r}\right) \neq \mu_{k}\left(n, t_{r}\right)$ for $j \neq k$, $j, k=1, \ldots, p$, then,

$$
a^{2}=\frac{1}{2} \sum_{j=1}^{p} y\left(\hat{\mu}_{j}\right) \prod_{\substack{k=1 \\ k \neq j}}^{p}\left(\mu_{j}-\mu_{k}\right)^{-1}+\frac{1}{4}\left(b^{(2)}-b^{2}\right) .
$$

For completeness we next mention the Dubrovin equations for the time variation of the Dirichlet eigenvalues of the Toda lattice.

Lemma 5.4. Assume Hypothesis 5.1 and suppose that $a, b$ satisfy (5.15) and (5.16). In addition, assume that the zeros $\mu_{j}\left(n, t_{r}\right), j=1, \ldots, p$, of $F_{p}\left(\cdot, n, t_{r}\right)$ remain distinct for all $\left(n, t_{r}\right) \in \mathbb{Z} \times \mathbb{R}$. Then,

$$
\begin{array}{r}
\frac{d}{d t_{r}} \mu_{j}\left(n, t_{r}\right)=-2 \tilde{F}_{r}\left(\mu_{j}\left(n, t_{r}\right), n, t_{r}\right) \frac{y\left(\hat{\mu}_{j}\left(n, t_{r}\right)\right)}{\prod_{\substack{\ell=1 \\
\ell \neq j}}^{p}\left(\mu_{j}\left(n, t_{r}\right)-\mu_{\ell}\left(n, t_{r}\right)\right)}, \\
j=1, \ldots, p,\left(n, t_{r}\right) \in \mathbb{Z} \times \mathbb{R} .
\end{array}
$$

When attempting to solve the Dubrovin system (5.42), it must be augmented with appropriate divisors $\mathcal{D}_{\hat{\mu}\left(n_{0}, t_{0, r}\right)} \in \operatorname{Sym}^{p} \mathcal{K}_{p}$ as initial conditions.

For the $t_{r}$-dependence of $F_{p}$ and $G_{p+1}$ one obtains the following result.

Lemma 5.5. Assume Hypothesis 5.1 and suppose that $a, b$ satisfy (5.15) and (5.16). In addition, let $\left(z, n, t_{r}\right) \in \mathbb{C} \times \mathbb{Z} \times \mathbb{R}$. Then,

$$
\begin{aligned}
F_{p, t_{r}} & =2\left(F_{p} \widetilde{G}_{r+1}-G_{p+1} \widetilde{F}_{r}\right), \\
G_{p+1, t_{r}} & =4 a^{2}\left(F_{p} \widetilde{F}_{r}^{+}-F_{p}^{+} \widetilde{F}_{r}\right) .
\end{aligned}
$$

In particular, (5.43) and (5.44) are equivalent to

$$
V_{p+1, t_{r}}=\left[\widetilde{V}_{r+1}, V_{p+1}\right] .
$$

It will be shown in Section 6 that Lemma 5.5 in conjunction with the fundamental identity (5.19) yields a first-order system of differential equations for $f_{\ell}, g_{\ell}, \ell=1, \ldots, p$, that serves as a pertinent substitute for the Dubrovin equations (5.42) even (in fact, especially) when some of the $\mu_{j}$ coincide.

As in the case of trace formulas, also Lemma 3.4 on nonspecial Dirichlet divisors $\mathcal{D}_{\hat{\mu}}$ and the linearization property of the Abel map when applied to $\mathcal{D}_{\underline{\hat{\mu}}}$ extends to the present time-dependent setting. For the latter fact we need to introduce a particular differential of the second kind, $\widetilde{\Omega}_{r}^{(2)}$, defined 
as follows. Let $\omega_{P_{\infty_{+}}, q}^{(2)}$ be the normalized Abelian differential of the second kind (i.e., with vanishing $a$-periods) with a single pole at $P_{\infty_{ \pm}}$of the form

$$
\omega_{P_{\infty_{ \pm}}, q}^{(2)}=\left(\zeta^{-2-q}+O(1)\right) d \zeta \text { near } P_{\infty_{ \pm}}, \quad q \in \mathbb{N}_{0} .
$$

Given the summation constants $\tilde{c}_{1}, \ldots, \tilde{c}_{r}$ in $\widetilde{F}_{r}$ (cf. (5.13)), we then define

$$
\widetilde{\Omega}_{r}^{(2)}=\sum_{q=0}^{r}(q+1) \tilde{c}_{r-q}\left(\omega_{P_{\infty_{+}}, q}^{(2)}-\omega_{P_{\infty_{-}, q}}^{(2)}\right), \quad \tilde{c}_{0}=1 .
$$

Since the differentials $\omega_{P_{\infty_{ \pm}}, q}^{(2)}$ were supposed to be normalized we have

$$
\int_{a_{j}} \widetilde{\Omega}_{r}^{(2)}=0, \quad j=1, \ldots, p
$$

Moreover, writing

$$
\omega_{j}=\left(\sum_{m=0}^{\infty} d_{j, m}\left(P_{\infty_{ \pm}}\right) \zeta^{m}\right) d \zeta= \pm\left(\sum_{m=0}^{\infty} d_{j, m}\left(P_{\infty_{+}}\right) \zeta^{m}\right) d \zeta \text { near } P_{\infty_{ \pm}}
$$

relation (A.20) yields for the vector of $b$-periods $\underline{U}_{r}^{(2)}$ of $\widetilde{\Omega}_{r}^{(2)}$,

$$
\begin{aligned}
& \widetilde{U}_{r}^{(2)}=\left(\widetilde{U}_{r, 1}^{(2)}, \ldots, \widetilde{U}_{r, p}^{(2)}\right), \\
& \widetilde{U}_{r, j}^{(2)}=\frac{1}{2 \pi i} \int_{b_{j}} \widetilde{\Omega}_{r}^{(2)}=2 \sum_{q=0}^{r} \tilde{c}_{r-q} d_{j, q}\left(P_{\infty_{+}}\right), \quad j=1, \ldots, p .
\end{aligned}
$$

The time-dependent analog of Lemma 3.4 then reads as follows.

Lemma 5.6. Assume Hypothesis 5.1 and suppose that $a, b$ satisfy (5.15) and (5.16). Let $\mathcal{D}_{\underline{\hat{\mu}}}, \underline{\hat{\mu}}=\left\{\hat{\mu}_{1}, \ldots, \hat{\mu}_{p}\right\} \in \operatorname{Sym}^{p}\left(\mathcal{K}_{p}\right)$, be the Dirichlet divisor of degree $p$ associate $\bar{d}$ with $a, b$, and $\phi$ defined according to (5.20), that is,

$$
\begin{array}{r}
\hat{\mu}_{j}\left(n, t_{r}\right)=\left(\mu_{j}\left(n, t_{r}\right),-G_{p+1}\left(\mu_{j}\left(n, t_{r}\right), n, t_{r}\right)\right) \in \mathcal{K}_{p}, \\
j=1, \ldots, p,\left(n, t_{r}\right) \in \mathbb{Z} \times \mathbb{R} .
\end{array}
$$

Then $\mathcal{D}_{\underline{\hat{\mu}}\left(n, t_{r}\right)}$ is nonspecial for all $\left(n, t_{r}\right) \in \mathbb{Z} \times \mathbb{R}$. Moreover, the Abel map linearizes the auxiliary divisor $\mathcal{D}_{\hat{\mu}}$ in the sense that

$$
\underline{\alpha}_{Q_{0}}\left(\mathcal{D}_{\hat{\hat{u}}\left(n, t_{r}\right)}\right)=\underline{\alpha}_{Q_{0}}\left(\mathcal{D}_{\underline{\hat{\mu}}\left(n_{0}, t_{0, r}\right)}\right)-\left(n-n_{0}\right) \underline{A}_{P_{\infty_{-}}}\left(P_{\infty_{+}}\right)-\left(t_{r}-t_{0, r}\right) \underline{\widetilde{U}}_{r}^{(2)}
$$

where $Q_{0} \in \mathcal{K}_{p}$ is a given base point and $\widetilde{U}_{r}^{(2)}$ is the vector of b-periods of the differential of the second kind $\widetilde{\Omega}_{r}^{(2)}$ introduced in (5.51). 
In addition, if $a, b \in L^{\infty}(\mathbb{Z} \times \mathbb{R})$, then there exists a constant $C_{\mu}>0$ such that

$$
\left|\mu_{j}\left(n, t_{r}\right)\right| \leq C_{\mu}, \quad j=1, \ldots, p,\left(n, t_{r}\right) \in \mathbb{Z} \times \mathbb{R} .
$$

Proof. We will prove that

$$
\begin{aligned}
& \psi\left(P, n, n_{0}, t_{r}, t_{0, r}\right)=C\left(n, t_{r}\right) \frac{\theta\left(\underline{z}\left(P, n, t_{r}\right)\right)}{\theta\left(\underline{z}\left(P, n_{0}, t_{0, r}\right)\right)} \\
& \times \exp \left(\left(n-n_{0}\right) \int_{Q_{0}}^{P} \omega_{P_{\infty_{+}}, P_{\infty_{-}}}^{(3)}+\left(t_{r}-t_{0, r}\right) \int_{Q_{0}}^{P} \widetilde{\Omega}_{r}^{(2)}\right),
\end{aligned}
$$

where

$$
\underline{z}\left(P, n, t_{r}\right)=\underline{A}_{Q_{0}}(P)-\underline{\alpha}_{Q_{0}}\left(\mathcal{D}_{\underline{\hat{\mu}}\left(n, t_{r}\right)}\right)+\Xi_{Q_{0}} .
$$

By Lemma 13.4 of [32] it suffices to show that the essential singularities at $P_{\infty_{ \pm}}$are equal. That is, by (5.25) we need to show that

$$
\begin{aligned}
\psi & \left.P, n_{0}, n_{0}, t_{r}, t_{0, r}\right) \\
& =\exp \left(\int_{t_{0, r}}^{t_{r}} d s\left(2 a\left(n_{0}, s\right) \widetilde{F}_{r}\left(z, n_{0}, t_{r}\right) \phi\left(P, n_{0}, t_{0, r}\right)+\widetilde{G}_{r+1}\left(z, n_{0}, s\right)\right)\right) \\
& =\exp \left( \pm\left(t_{r}-t_{0, r}\right) \sum_{k=0}^{r} c_{r-k} \zeta^{-k-1}+O(1)\right) \text { for } P \text { near } P_{\infty_{ \pm}} \cdot
\end{aligned}
$$

Using (5.22) and (5.43) one obtains

$$
\psi\left(P, n_{0}, n_{0}, t_{r}, t_{0, r}\right)=\left(\frac{F_{p}\left(z, n_{0}, t_{r}\right)}{F_{p}\left(z, n_{0}, t_{0, r}\right)}\right)^{1 / 2} \exp \left(y \int_{t_{0, r}}^{t_{r}} d s \frac{\widetilde{F}_{r}\left(z, n_{0}, s\right)}{F_{p}\left(z, n_{0}, s\right)}\right)
$$

and the desired asymptotics follow from Theorem C.1, which tells us that

$$
\frac{y}{F_{p}\left(z, n_{0}, s\right)} \widehat{F}_{k}\left(z, n_{0}, s\right)= \pm \zeta^{-k-1}+O(1) \text { for } P \text { near } P_{\infty_{ \pm}}
$$

together with (2.23).

Again the analog of Remark 3.5 applies in the present time-dependent context. 


\section{The algebro-geometric Toda hierarchy initial value problem}

In this section we consider the algebro-geometric Toda hierarchy initial value problem (5.2), (5.3) with complex-valued initial data. For a generic set of initial data we will prove unique solvability of the initial value problem globally in time.

While it is natural in the special self-adjoint case to base the solution of the algebro-geometric initial value problem on the Dubrovin equations (5.42) (and the trace formula (5.40) for $b$ and formula (5.41) for $a^{2}$ ), this strategy meets with difficulties in the non-self-adjoint case as Dirichlet eigenvalues $\hat{\mu}_{j}$ may now collide on $\mathcal{K}_{p}$ and hence the denominator of (5.42) can blow up. Hence, we will develop an alternative strategy based on the use of elementary symmetric functions of the variables $\left\{\mu_{j}\right\}_{j=1, \ldots, p}$ in this section, which can accommodate collisions of $\hat{\mu}_{j}$. In short, our strategy will be as follows:

(i) Replace the first-order autonomous Dubrovin system (5.42) of differential equations in $t_{r}$ for the Dirichlet eigenvalues $\mu_{j}\left(n, t_{r}\right), j=1, \ldots, p$, augmented by appropriate initial conditions, by the first-order autonomous system $(6.27),(6.28)$ for the coefficients $f_{j}, j=1, \ldots, p, g_{j}, j=1, \ldots, p-1$, and $g_{p}+f_{p+1}$ with respect to $t_{r}$. (We note that $f_{j}, j=1, \ldots, p$, are symmetric functions of $\mu_{1}, \ldots, \mu_{p}$.) Solve this first-order autonomous system in some time interval $\left(t_{0, r}-T_{0}, t_{0, r}+T_{0}\right)$ under appropriate initial conditions at $\left(n_{0}, t_{0, r}\right)$ derived from an initial (nonspecial) Dirichlet divisor $\mathcal{D}_{\underline{\hat{\mu}}\left(n_{0}, t_{0, r}\right)}$.

(ii) Use the stationary algorithm derived in Section 4 to extend the solution of step $(i)$ from $\left\{n_{0}\right\} \times\left(t_{0, r}-T_{0}, t_{0, r}+T_{0}\right)$ to $\mathbb{Z} \times\left(t_{0, r}-T_{0}, t_{0, r}+T_{0}\right)$ (cf. Lemma 6.2).

(iii) Prove consistency of this approach, that is, show that the discrete algorithm of Section 4 is compatible with the time-dependent Lax and zero-curvature equations in the sense that first solving the autonomous system (6.27), (6.28) and then applying the discrete algorithm, or first applying the discrete algorithm and then solving the autonomous system (6.27), (6.28) yields the same result whenever the same endpoint $\left(n, t_{r}\right)$ is reached (cf. the discussion following Lemma 6.2 and the subsequent Lemma 6.3 and Theorem 6.4).

(iv) Prove that there is a dense set of initial conditions of full measure for which this strategy yields global solutions of the algebro-geometric Toda hierarchy initial value problem (cf. Lemma 6.5 and Theorem 6.6).

To set up this formalism we need some preparations. From the outset we make the following assumption. 
Hypothesis 6.1. Suppose that

$$
a, b \in \mathbb{C}^{\mathbb{Z}} \text { and } a(n) \neq 0 \text { for all } n \in \mathbb{Z},
$$

and assume that $a, b$ satisfy the pth stationary Toda system (2.20). In addition, suppose that the hyperelliptic curve $\mathcal{K}_{p}$ in (3.1) is nonsingular.

Assuming Hypothesis 6.1, we consider the polynomials $F_{p}, G_{p+1}, \widetilde{F}_{r}$, and $\widetilde{G}_{r+1}$ given by (5.11)-(5.14) for fixed $p, r \in \mathbb{N}_{0}$. Here $f_{\ell}, \tilde{f}_{s}, g_{\ell}$, and $\tilde{g}_{s}$, $\ell=0, \ldots, p, s=0, \ldots, r$, are defined as in (2.4)-(2.6) with appropriate sets of summation constants.

Our aim will be to find an autonomous first-order system of ordinary differential equations with respect to $t_{r}$ of $f_{\ell}$ and $g_{\ell}$ rather than $\mu_{j}$. Indeed, we will take the coupled system of differential equations (5.43), (5.44), properly rewritten next, as our point of departure. In order to turn (5.43), (5.44) into a system of first-order ordinary differential equations for $f_{\ell}$ and $g_{\ell}$, we first need to express $f_{\ell}^{+}, \tilde{f}_{s}, \tilde{g}_{s}$, and $\tilde{f}_{s}^{+}$in terms of $f_{\ell}$ and $g_{\ell}$ as follows.

Using (2.9), (2.23), (2.25), and Theorem C.1 one infers

$$
\begin{aligned}
& \widetilde{F}_{r}(z)=\sum_{s=0}^{r} \tilde{f}_{r-s} z^{s}=\sum_{s=0}^{r} \tilde{c}_{r-s} \widehat{F}_{s}(z), \\
& \widehat{F}_{\ell}(z)=\sum_{k=0}^{\ell} \hat{f}_{\ell-k} z^{k}, \quad \hat{f}_{0}=1, \quad \hat{f}_{\ell}=\sum_{k=0}^{\ell \wedge p} \hat{c}_{\ell-k}(\underline{E}) f_{k}, \quad \ell \in \mathbb{N}_{0},
\end{aligned}
$$

where $m \wedge n=\min \{m, n\}$ and $\hat{c}_{\ell}(\underline{E})$ has been introduced in (C.4). Hence one obtains

$$
\tilde{f}_{0}=1, \quad \tilde{f}_{s}=\mathcal{F}_{1, s}\left(f_{1}, \ldots, f_{p}\right), \quad s=1, \ldots, r
$$

where $\mathcal{F}_{1, s}, s=1, \ldots, r$, are polynomials in $p$ variables.

Next, using (2.9), (2.24), (2.26), and Theorem C.1 one concludes

$$
\widetilde{G}_{r+1}(z)=-z^{r+1}+\sum_{s=0}^{r} \tilde{g}_{r-s} z^{s}+\tilde{f}_{r+1}=\sum_{s=1}^{r+1} \tilde{c}_{r+1-s} \widehat{G}_{s}(z),
$$

$$
\widehat{G}_{0}(z)=\left.G_{0}(z)\right|_{c_{1}=0}=0, \quad \widehat{G}_{1}(z)=G_{1}(z)=-z-b
$$$$
\widehat{G}_{\ell+1}(z)=\left.G_{\ell+1}(z)\right|_{c_{k}=0, k=1, \ldots, \ell}=-z^{\ell+1}+\sum_{k=0}^{\ell} \hat{g}_{\ell-k} z^{k}+\hat{f}_{\ell+1}, \quad \ell \in \mathbb{N},
$$

(6.6) $\hat{g}_{0}=0, \quad \hat{g}_{\ell}=\sum_{k=0}^{\ell \wedge p} \hat{c}_{\ell-k}(\underline{E})\left(g_{k}+f_{p+1} \delta_{p, k}\right)-\hat{c}_{\ell+1}(\underline{E}), \quad \ell \in \mathbb{N}$. 
Hence one concludes

$$
\tilde{g}_{0}=-\tilde{c}_{1}, \quad \tilde{g}_{s}=\mathcal{F}_{2, s}\left(f_{1}, \ldots, f_{p}, g_{1}, \ldots, g_{p-1},\left(g_{p}+f_{p+1}\right)\right), \quad s=1, \ldots, r
$$

where $\mathcal{F}_{2, s}, s=1, \ldots, r$, are polynomials in $2 p$ variables. We also recall (cf. (2.18)) that $f_{p+1}$ is a lattice constant, that is,

$$
f_{p+1}=f_{p+1}^{-} \text {. }
$$

Next we invoke the fundamental identity (2.34) in the form

$$
-4 a^{2} F_{p}^{+}=\frac{R_{2 p+2}-G_{p+1}^{2}}{F_{p}} .
$$

While (6.9) at this point only holds in the stationary context, we will use it later on also in the $t_{r}$-dependent context and verify after the time-dependent solutions of (5.2), (5.3) have been obtained that (6.9) indeed is valid for all $\left(n, t_{r}\right) \in \mathbb{Z} \times \mathbb{R}$. A comparison of powers of $z$ in (6.9) then yields

$$
\begin{aligned}
& 4 a^{2} f_{0}^{+}=-2 g_{1}-2 c_{2}, \\
& 4 a^{2} f_{\ell}^{+}=\mathcal{F}_{3, \ell}\left(f_{1}, \ldots, f_{p}, g_{1}, \ldots, g_{p-1},\left(g_{p}+f_{p+1}\right)\right), \quad \ell=1, \ldots, p,
\end{aligned}
$$

where $\mathcal{F}_{3, \ell}, \ell=1, \ldots, p$, are polynomials in $2 p$ variables.

Finally, combining (6.2), (6.3), (6.9), and (6.10), one obtains

$$
\begin{aligned}
& 4 a^{2} \tilde{f}_{0}^{+}=-2 g_{1}-2 c_{2}, \\
& 4 a^{2} \tilde{f}_{s}^{+}=\mathcal{F}_{4, s}\left(f_{1}, \ldots, f_{p}, g_{1}, \ldots, g_{p-1},\left(g_{p}+f_{p+1}\right)\right), \quad s=1, \ldots, r,
\end{aligned}
$$

where $\mathcal{F}_{4, s}, s=1, \ldots, 3$, are polynomials in $2 p$ variables.

We emphasize that also the Dubrovin equations (5.42) require an analogous rewriting of $\widetilde{F}_{r}$ in terms of (symmetric functions of) $\mu_{j}$ in order to represent a first-order system of differential equations for $\mu_{j}, j=1, \ldots, p$.

Next, we make the transition to the algebro-geometric initial value problem (5.2), (5.3). We introduce a deformation (time) parameter $t_{r} \in \mathbb{R}$ in $a=a\left(t_{r}\right)$ and $b=b\left(t_{r}\right)$ and hence obtain $t_{r}$-dependent quantities $f_{\ell}=f_{\ell}\left(t_{r}\right)$, $g_{\ell}=g_{\ell}\left(t_{r}\right), F_{p}(z)=F_{p}\left(z, t_{r}\right), G_{p+1}(z)=G_{p+1}\left(z, t_{r}\right)$, etc. At a fixed initial time $t_{0, r} \in \mathbb{R}$ we require that

$$
\left.(a, b)\right|_{t_{r}=t_{0, r}}=\left(a^{(0)}, b^{(0)}\right)
$$

where $a^{(0)}=a\left(\cdot, t_{0, r}\right), b^{(0)}=b\left(\cdot, t_{0, r}\right)$ satisfy the $p$ th stationary Toda equation (2.20) as in (6.1)-(6.11). As discussed in Section 4, in order to guarantee that the stationary solutions $(6.12)$ can be constructed for all $n \in \mathbb{Z}$ one starts from a particular divisor

$$
\mathcal{D}_{\underline{\hat{\mu}}\left(n_{0}, t_{0, r}\right)} \in \mathcal{M}_{0}
$$


where $\underline{\hat{\mu}}\left(n_{0}, t_{0, r}\right)$ is of the form

$$
\begin{aligned}
& \underline{\hat{\mu}}\left(n_{0}, t_{0, r}\right)=\left\{\hat{\mu}_{1}\left(n_{0}, t_{0, r}\right), \ldots, \hat{\mu}_{p}\left(n_{0}, t_{0, r}\right)\right\} \\
& =\{\underbrace{\hat{\mu}_{1}\left(n_{0}, t_{0, r}\right), \ldots, \hat{\mu}_{1}\left(n_{0}, t_{0, r}\right)}_{p_{1}\left(n_{0}, t_{0, r}\right) \text { times }}, \ldots, \\
& \ldots, \underbrace{\hat{\mu}_{q\left(n_{0}, t_{0, r}\right)}\left(n_{0}, t_{0, r}\right), \ldots, \hat{\mu}_{q\left(n_{0}, t_{0, r}\right)}\left(n_{0}, t_{0, r}\right)}_{p_{q\left(n_{0}, t_{0, r}\right)}\left(n_{0}, t_{0, r}\right) \text { times }}\}
\end{aligned}
$$

with

$$
\begin{aligned}
& \hat{\mu}_{k}\left(n_{0}, t_{0, r}\right)=\left(\mu_{k}\left(n_{0}, t_{0, r}\right), y\left(\hat{\mu}_{k}\left(n_{0}, t_{0, r}\right)\right)\right), \\
& \mu_{k}\left(n_{0}, t_{0, r}\right) \neq \mu_{k^{\prime}}\left(n_{0}, t_{0, r}\right) \text { for } k \neq k^{\prime}, k, k^{\prime}=1, \ldots, q\left(n_{0}, t_{0, r}\right),
\end{aligned}
$$

and

$$
p_{k}\left(n_{0}, t_{0, r}\right) \in \mathbb{N}, k=1, \ldots, q\left(n_{0}, t_{0, r}\right), \quad \sum_{k=1}^{q\left(n_{0}, t_{0, r}\right)} p_{k}\left(n_{0}, t_{0, r}\right)=p
$$

Next we recall

$$
\begin{aligned}
& F_{p}\left(z, n_{0}, t_{0, r}\right)=\sum_{\ell=0}^{p} f_{p-\ell}\left(n_{0}, t_{0, r}\right) z^{\ell}=\prod_{k=1}^{q\left(n_{0}, t_{0, r}\right)}\left(z-\mu_{k}\left(n_{0}, t_{0, r}\right)\right)^{p_{k}\left(n_{0}, t_{0, r}\right)}, \\
& T_{p-1}\left(z, n_{0}, t_{0, r}\right)=-F_{p}\left(z, n_{0}, t_{0, r}\right) \\
& \times \sum_{k=1}^{q\left(n_{0}, t_{0, r}\right)} \sum_{\ell=0}^{p_{k}\left(n_{0}, t_{0, r}\right)-1} \frac{\left.\left(d^{\ell}\left(R_{2 p+2}(\zeta)^{1 / 2}\right) / d \zeta^{\ell}\right)\right|_{\zeta=\mu_{k}\left(n_{0}, t_{0, r}\right)}}{\ell !\left(p_{k}\left(n_{0}, t_{0, r}\right)-\ell-1\right) !} \\
& \times\left(\frac { d ^ { p _ { k } ( n _ { 0 } , t _ { 0 , r } ) - \ell - 1 } } { d \zeta ^ { p _ { k } ( n _ { 0 } , t _ { 0 , r } ) - \ell - 1 } } \left((z-\zeta)^{-1}\right.\right. \\
& \left.\left.\times \prod_{k^{\prime}=1, k^{\prime} \neq k}^{q\left(n_{0}, t_{0, r}\right)}\left(\zeta-\mu_{k^{\prime}}\left(n_{0}, t_{0, r}\right)\right)^{-p_{k^{\prime}}\left(n_{0}, t_{0, r}\right)}\right)\right)\left.\right|_{\zeta=\mu_{k}\left(n_{0}, t_{0, r}\right)}, \\
& b\left(n_{0}, t_{0, r}\right)=\frac{1}{2} \sum_{m=0}^{2 p+1} E_{m}-\sum_{k=1}^{q\left(n_{0}, t_{0, r}\right)} p_{k}\left(n_{0}, t_{0, r}\right) \mu_{k}\left(n_{0}, t_{0, r}\right) \\
& G_{p+1}\left(z, n_{0}, t_{0, r}\right)=-z^{p+1}+\sum_{\ell=0}^{p} g_{p-\ell}\left(n_{0}, t_{0, r}\right) z^{\ell}+f_{p+1}\left(t_{0, r}\right), \\
& =-\left(z-b\left(n_{0}, t_{0, r}\right)\right) F_{p}\left(z, n_{0}, t_{0, r}\right)+T_{p-1}\left(z, n_{0}, t_{0, r}\right) \text {. }
\end{aligned}
$$


Here the sign of the square root in (6.18) is chosen as usual by

$$
\begin{array}{r}
\hat{\mu}_{k}\left(n_{0}, t_{0, r}\right)=\left(\mu_{k}\left(n_{0}, t_{0, r}\right), y\left(\hat{\mu}_{k}\left(n_{0}, t_{0, r}\right)\right)\right) \\
=\left(\mu_{k}\left(n_{0}, t_{0, r}\right), R_{2 p+2}\left(\mu_{k}\left(n_{0}, t_{0, r}\right)\right)^{1 / 2}\right) \\
=\left(\mu_{k}\left(n_{0}, t_{0, r}\right),-G_{p+1}\left(\mu_{k}\left(n_{0}, t_{0, r}\right), n_{0}, t_{0, r}\right)\right), \\
\quad k=1, \ldots, q\left(n_{0}, t_{0, r}\right) .
\end{array}
$$

By (6.17) one concludes that (6.14) uniquely determines $F_{p}\left(z, n_{0}, t_{0, r}\right)$ and hence

$$
f_{1}\left(n_{0}, t_{0, r}\right), \ldots, f_{p}\left(n_{0}, t_{0, r}\right) \text {. }
$$

By (6.18)-(6.22) one concludes that also $G_{p+1}\left(z, n_{0}, t_{0, r}\right)$ and hence

$$
g_{1}\left(n_{0}, t_{0, r}\right), \ldots, g_{p-1}\left(n_{0}, t_{0, r}\right), g_{p}\left(n_{0}, t_{0, r}\right)+f_{p+1}\left(t_{0, r}\right)
$$

are uniquely determined by the initial divisor $\mathcal{D}_{\underline{\hat{\mu}}\left(n_{0}, t_{0, r}\right)}$ in $(6.13)$.

Summing up the discussion in (6.2)-(6.23), we can transform the differential equations

$$
\begin{aligned}
& F_{p, t_{r}}\left(z, n_{0}, t_{r}\right)=2( F_{p}\left(z, n_{0}, t_{r}\right) \widetilde{G}_{r+1}\left(z, n_{0}, t_{r}\right) \\
&\left.-G_{p+1}\left(z, n_{0}, t_{r}\right) \widetilde{F}_{r}\left(z, n_{0}, t_{r}\right)\right), \\
& G_{p+1, t_{r}}\left(z, n_{0}, t_{r}\right)=4 a\left(n_{0}, t_{r}\right)^{2}\left(F_{p}\left(z, n_{0}, t_{r}\right) \widetilde{F}_{r}^{+}\left(z, n_{0}, t_{r}\right)\right. \\
&\left.-F_{p}^{+}\left(z, n_{0}, t_{r}\right) \widetilde{F}_{r}\left(z, n_{0}, t_{r}\right)\right)
\end{aligned}
$$

subject to the constraint

$$
-4 a^{2} F_{p}^{+}\left(z, n_{0}, t_{r}\right)=\frac{R_{2 p+2}(z)-G_{p+1}\left(z, n_{0}, t_{r}\right)^{2}}{F_{p}\left(z, n_{0}, t_{r}\right)}
$$

and associated with an initial divisor $\mathcal{D}_{\hat{\underline{\mu}}\left(n_{0}, t_{0, r}\right)}$ in (6.13) into the following autonomous first-order system of ordinary differential equations (for fixed $n=n_{0}$ ),

$$
\begin{aligned}
& f_{j, t_{r}}=\mathcal{F}_{j}\left(f_{1}, \ldots, f_{p}, g_{1}, \ldots, g_{p-1}, g_{p}+f_{p+1}\right), \quad j=1, \ldots, p, \\
& g_{j, t_{r}}=\mathcal{G}_{j}\left(f_{1}, \ldots, f_{p}, g_{1}, \ldots, g_{p-1}, g_{p}+f_{p+1}\right), \quad j=1, \ldots, p-1, \\
& \left(g_{p}+f_{p+1}\right)_{t_{r}}=\mathcal{G}_{p}\left(f_{1}, \ldots, f_{p}, g_{1}, \ldots, g_{p-1}, g_{p}+f_{p+1}\right)
\end{aligned}
$$

with initial condition

$$
\begin{aligned}
& f_{j}\left(n_{0}, t_{0, r}\right), \quad j=1, \ldots, p \\
& g_{j}\left(n_{0}, t_{0, r}\right), \quad j=1, \ldots, p-1, \\
& g_{p}\left(n_{0}, t_{0, r}\right)+f_{p+1}\left(t_{0, r}\right)
\end{aligned}
$$


where $\mathcal{F}_{j}, \mathcal{G}_{j}, j=1, \ldots, p$, are polynomials in $2 p$ variables. As just discussed, the initial conditions (6.28) are uniquely determined by the initial divisor $\mathcal{D}_{\underline{\hat{\mu}}\left(n_{0}, t_{0, r}\right)}$ in $(6.13)$.

Being autonomous with polynomial right-hand sides, there exists a $T_{0}>0$, such that the first-order initial value problem (6.27), (6.28) has a unique solution

$$
\begin{aligned}
& f_{j}=f_{j}\left(n_{0}, t_{r}\right), \quad j=1, \ldots, p, \\
& g_{j}=g_{j}\left(n_{0}, t_{r}\right), \quad j=1, \ldots, p-1, \\
& g_{p}+f_{p+1}=g_{p}\left(n_{0}, t_{r}\right)+f_{p+1}\left(t_{r}\right) \\
& \text { for all } t_{r} \in\left(t_{0, r}-T_{0}, t_{0, r}+T_{0}\right)
\end{aligned}
$$

(cf., e.g., [35, Sect. III.10]). Given the solution (6.29), we next introduce the following quantities (where $t_{r} \in\left(t_{0, r}-T_{0}, t_{0, r}+T_{0}\right)$ ):

$$
\begin{aligned}
& F_{p}\left(z, n_{0}, t_{r}\right)=\sum_{\ell=0}^{p} f_{p-\ell}\left(n_{0}, t_{r}\right) z^{\ell}=\prod_{k=1}^{q\left(n_{0}, t_{r}\right)}\left(z-\mu_{k}\left(n_{0}, t_{r}\right)\right)^{p_{k}\left(n_{0}, t_{r}\right)} \\
& T_{p-1}\left(z, n_{0}, t_{r}\right)=-F_{p}\left(z, n_{0}, t_{r}\right) \\
& \times \sum_{k=1}^{q\left(n_{0}, t_{r}\right)} \sum_{\ell=0}^{p_{k}\left(n_{0}, t_{r}\right)-1} \frac{\left.\left(d^{\ell}\left(R_{2 p+2}(\zeta)^{1 / 2}\right) / d \zeta^{\ell}\right)\right|_{\zeta=\mu_{k}\left(n_{0}, t_{r}\right)}}{\ell !\left(p_{k}\left(n_{0}, t_{r}\right)-\ell-1\right) !} \\
& \times\left(\frac { d ^ { p _ { k } ( n _ { 0 } , t _ { r } ) - \ell - 1 } } { d \zeta ^ { p _ { k } ( n _ { 0 } , t _ { r } ) - \ell - 1 } } \left((z-\zeta)^{-1}\right.\right. \\
& \left.\left.\times \prod_{k^{\prime}=1, k^{\prime} \neq k}^{q\left(n_{0}, t_{r}\right)}\left(\zeta-\mu_{k^{\prime}}\left(n_{0}, t_{r}\right)\right)^{-p_{k^{\prime}}\left(n_{0}, t_{r}\right)}\right)\right)\left.\right|_{\zeta=\mu_{k}\left(n_{0}, t_{r}\right)}, \\
& b\left(n_{0}, t_{r}\right)=\frac{1}{2} \sum_{m=0}^{2 p+1} E_{m}-\sum_{k=1}^{q\left(n_{0}, t_{r}\right)} p_{k}\left(n_{0}, t_{r}\right) \mu_{k}\left(n_{0}, t_{r}\right), \\
& G_{p+1}\left(z, n_{0}, t_{r}\right)=-z^{p+1}+\sum_{\ell=0}^{p} g_{p-\ell}\left(n_{0}, t_{r}\right) z^{\ell}+f_{p+1}\left(t_{r}\right) \\
& =-\left(z-b\left(n_{0}, t_{r}\right)\right) F_{p}\left(z, n_{0}, t_{r}\right)+T_{p-1}\left(z, n_{0}, t_{r}\right) .
\end{aligned}
$$

In particular, this leads to the divisor

$$
\mathcal{D}_{\underline{\hat{\mu}}\left(n_{0}, t_{r}\right)} \in \operatorname{Sym}^{p}\left(\mathcal{K}_{p}\right)
$$

and the sign of the square root in (6.31) is chosen as usual by

$$
\begin{aligned}
\hat{\mu}_{k}\left(n_{0}, t_{r}\right) & =\left(\mu_{k}\left(n_{0}, t_{r}\right),-G_{p+1}\left(\mu_{k}\left(n_{0}, t_{r}\right), n_{0}, t_{r}\right)\right) \\
& =\left(\mu_{k}\left(n_{0}, t_{r}\right), R_{2 p+2}\left(\mu_{k}\left(n_{0}, t_{r}\right)\right)^{1 / 2}\right), \quad k=1, \ldots, q\left(n_{0}, t_{r}\right),
\end{aligned}
$$


and

$$
\begin{aligned}
& \underline{\hat{\mu}}\left(n_{0}, t_{r}\right)=\left\{\mu_{1}\left(n_{0}, t_{r}, \ldots, \mu_{p}\left(n_{0}, t_{r}\right)\right\}\right. \\
& \quad=\{\underbrace{\mu_{1}\left(n_{0}, t_{r}\right), \ldots, \mu_{1}\left(n_{0}, t_{r}\right)}_{p_{1}\left(n_{0}, t_{r}\right) \text { times }}, \ldots, \underbrace{\mu_{q\left(n_{0}, t_{r}\right)}\left(n_{0}, t_{r}\right), \ldots, \mu_{q\left(n_{0}, t_{r}\right)}\left(n_{0}, t_{r}\right)}_{p_{q\left(n_{0}, t_{r}\right)}\left(n_{0}, t_{r}\right) \text { times }}\}
\end{aligned}
$$

with

$$
\mu_{k}\left(n_{0}, t_{r}\right) \neq \mu_{k^{\prime}}\left(n_{0}, t_{r}\right) \text { for } k \neq k^{\prime}, k, k^{\prime}=1, \ldots, q\left(n_{0}, t_{r}\right)
$$

and

$$
p_{k}\left(n_{0}, t_{r}\right) \in \mathbb{N}, k=1, \ldots, q\left(n_{0}, t_{r}\right), \quad \sum_{k=1}^{q\left(n_{0}, t_{r}\right)} p_{k}\left(n_{0}, t_{r}\right)=p .
$$

By construction (cf. (6.35)), the divisor $\mathcal{D}_{\underline{\hat{\mu}}\left(n_{0}, t_{r}\right)}$ is nonspecial for all $t_{r} \in$ $\left(t_{0, r}-T_{0}, t_{0, r}+T_{0}\right)$.

In exactly the same manner as in (4.28)-(4.31) one then infers that $F_{p}\left(\cdot, n_{0}, t_{r}\right)$ divides $R_{2 p+2}-G_{p+1}^{2}$ (since $t_{r}$ is just a fixed additional parameter). Moreover, arguing as in (4.32)-(4.38) we now assume that the polynomial

$$
R_{2 p+2}(z)-G_{p+1}\left(z, n_{0}, t_{r}\right)^{2} \underset{z \rightarrow \infty}{=} O\left(z^{2 p}\right)
$$

is precisely of maximal order $2 p$ for all $t_{r} \in\left(t_{0, r}-T_{0}, t_{0, r}+T_{0}\right)$. One then obtains

$$
\begin{gathered}
R_{2 p+2}(z)-G_{p+1}\left(z, n_{0}, t_{r}\right)^{2}=-4 a\left(n_{0}, t_{r}\right)^{2} F_{p}\left(z, n_{0}, t_{r}\right) F_{p}\left(z, n_{0}+1, t_{r}\right), \\
\left(z, t_{r}\right) \in \mathbb{C} \times\left(t_{0, r}-T_{0}, t_{0, r}+T_{0}\right),
\end{gathered}
$$

where we introduced the coefficient $a\left(n_{0}, t_{r}\right)^{2}$ to make $F_{p}\left(\cdot, n_{0}+1, t_{r}\right)$ a monic polynomial of degree $p$. As in Section 4 , the assumption that the polynomial $F_{p}\left(\cdot, n_{0}+1, t_{r}\right)$ is precisely of order $p$ is implied by the hypothesis that

$$
\mathcal{D}_{\underline{\hat{\mu}}\left(n_{0}, t_{r}\right)} \in \mathcal{M}_{0} \text { for all } t_{r} \in\left(t_{0, r}-T_{0}, t_{0, r}+T_{0}\right),
$$

a point we will revisit later (cf. Lemma 6.5). Given (6.40), we obtain consistency with (6.9) for $n=n_{0}$ and $t_{r} \in\left(t_{0, r}-T_{0}, t_{0, r}+T_{0}\right)$.

The explicit formula for $a\left(n_{0}, t_{r}\right)^{2}$ then reads (for $t_{r} \in\left(t_{0, r}-T_{0}, t_{0, r}+T_{0}\right)$ )

$$
\begin{aligned}
a\left(n_{0}, t_{r}\right)^{2}= & \frac{1}{2} \sum_{k=1}^{q\left(n_{0}, t_{r}\right)} \frac{\left.\left(d^{\ell}\left(R_{2 p+2}(z)^{1 / 2}\right) / d z^{\ell}\right)\right|_{z=\mu_{k}\left(n_{0}, t_{r}\right)}}{\left(p_{k}\left(n_{0}, t_{r}\right)-1\right) !} \\
& \times \prod_{k^{\prime}=1, k^{\prime} \neq k}^{q\left(n_{0}, t_{r}\right)}\left(\mu_{k}\left(n_{0}, t_{r}\right)-\mu_{k^{\prime}}\left(n_{0}, t_{r}\right)\right)^{-p_{k}\left(n_{0}, t_{r}\right)} \\
& +\frac{1}{4}\left(b^{(2)}\left(n_{0}, t_{r}\right)-b\left(n_{0}, t_{r}\right)^{2}\right) .
\end{aligned}
$$


Here and in the following we use the abbreviation

$$
b^{(2)}\left(n, t_{r}\right)=\frac{1}{2} \sum_{m=0}^{2 p+1} E_{m}^{2}-\sum_{k=1}^{q\left(n, t_{r}\right)} p_{k}\left(n, t_{r}\right) \mu_{k}\left(n, t_{r}\right)^{2}
$$

for appropriate ranges of $\left(n, t_{r}\right) \in \mathbb{N} \times \mathbb{R}$.

With (6.30)-(6.43) in place, we can now apply the stationary formalism as summarized in Theorem 4.4, subject to the additional hypothesis (6.41), for each fixed $t_{r} \in\left(t_{0, r}-T_{0}, t_{0, r}+T_{0}\right)$. This yields, in particular, the quantities

$$
F_{p}, G_{p+1}, a, b \text {, and } \underline{\hat{\mu}} \text { for }\left(n, t_{r}\right) \in \mathbb{Z} \times\left(t_{0, r}-T_{0}, t_{0, r}+T_{0}\right),
$$

which are of the form (6.30)-(6.43), replacing the fixed $n_{0} \in \mathbb{Z}$ by an arbitrary $n \in \mathbb{Z}$. In addition, one has the following fundamental identities (cf. (4.54), (4.59), (4.62), and (4.63)), which we summarize in the following result.

Lemma 6.2. Assume Hypothesis 6.1 and condition (6.41). Then the following relations are valid:

$$
\begin{array}{r}
R_{2 p+2}-G_{p+1}^{2}+4 a^{2} F_{p} F_{p}^{+}=0, \\
2\left(z-b^{+}\right) F_{p}^{+}+G_{p+1}^{+}+G_{p+1}=0, \\
2 a^{2} F_{p}^{+}-2\left(a^{-}\right)^{2} F_{p}^{-}+(z-b)\left(G_{p+1}-G_{p+1}^{-}\right)=0 \\
2\left(z-b^{+}\right) F_{p}^{+}-2(z-b) F_{p}+G_{p+1}^{+}-G_{p+1}^{-}=0 \\
\text { on } \mathbb{C} \times \mathbb{Z} \times\left(t_{0, r}-T_{0}, t_{0, r}+T_{0}\right)
\end{array}
$$

and hence the stationary part, (5.9), of the algebro-geometric initial value problem holds,

$$
U V_{p+1}-V_{p+1}^{+} U=0 \text { on } \mathbb{C} \times \mathbb{Z} \times\left(t_{0, r}-T_{0}, t_{0, r}+T_{0}\right) .
$$

In particular, Lemmas 3.2-3.4 apply.

Lemma 6.2 now raises the following important consistency issue: On one hand, one can solve the initial value problem (6.27), (6.28) at $n=n_{0}$ in some interval $t_{r} \in\left(t_{0, r}-T_{0}, t_{0, r}+T_{0}\right)$, and then extend the quantities $F_{p}, G_{p+1}$ to all $\mathbb{C} \times \mathbb{Z} \times\left(t_{0, r}-T_{0}, t_{0, r}+T_{0}\right)$ using the stationary algorithm summarized in Theorem 4.4 as just recorded in Lemma 6.2. On the other hand, one can solve the initial value problem (6.27), (6.28) at $n=n_{1}, n_{1} \neq n_{0}$, in some interval $t_{r} \in\left(t_{0, r}-T_{1}, t_{0, r}+T_{1}\right)$ with the initial condition obtained by applying the discrete algorithm to the quantities $F_{p}, G_{p+1}$ starting at $\left(n_{0}, t_{0, r}\right)$ and ending at $\left(n_{1}, t_{0, r}\right)$. Consistency then requires that the two approaches yield the same result at $n=n_{1}$ for $t_{r}$ in some open neighborhood of $t_{0, r}$. 
Equivalently, and pictorially speaking, envisage a vertical $t_{r}$-axis and a horizontal $n$-axis. Then, consistency demands that first solving the initial value problem (6.27), (6.28) at $n=n_{0}$ in some $t_{r}$-interval around $t_{0, r}$ and using the stationary algorithm to extend $F_{p}, G_{p+1}$ horizontally to $n=n_{1}$ and the same $t_{r}$-interval around $t_{0, r}$, or first applying the stationary algorithm starting at $\left(n_{0}, t_{0, r}\right)$ to extend $F_{p}, G_{p+1}$ horizontally to $\left(n_{1}, t_{0, r}\right)$ and then solving the initial value problem (6.27), (6.28) at $n=n_{1}$ in some $t_{r}$-interval around $t_{0, r}$ should produce the same result at $n=n_{1}$ in a sufficiently small open $t_{r}$ interval around $t_{0, r}$.

To settle this consistency issue, we will prove the following result. To this end we find it convenient to replace the initial value problem (6.27), (6.28) by the original $t_{r}$-dependent zero-curvature equation (5.8), $U_{t_{r}}+U \widetilde{V}_{r+1}-$ $\widetilde{V}_{r+1}^{+} U=0$ on $\mathbb{C} \times \mathbb{Z} \times\left(t_{0, r}-T_{0}, t_{0, r}+T_{0}\right)$.

Lemma 6.3. Assume Hypothesis 6.1 and condition (6.41). Moreover, suppose that (6.24)-(6.26) hold on $\mathbb{C} \times\left\{n_{0}\right\} \times\left(t_{0, r}-T_{0}, t_{0, r}+T_{0}\right)$.

Then (6.24)-(6.26) hold on $\mathbb{C} \times \mathbb{Z} \times\left(t_{0, r}-T_{0}, t_{0, r}+T_{0}\right)$, that is,

$$
\begin{aligned}
& F_{p, t_{r}}\left(z, n, t_{r}\right)=2\left(F_{p}\left(z, n, t_{r}\right) \widetilde{G}_{r+1}\left(z, n, t_{r}\right)\right. \\
&\left.-G_{p+1}\left(z, n, t_{r}\right) \widetilde{F}_{r}\left(z, n, t_{r}\right)\right), \\
& G_{p+1, t_{r}}\left(z, n, t_{r}\right)=4 a\left(n, t_{r}\right)^{2}\left(F_{p}\left(z, n, t_{r}\right) \widetilde{F}_{r}^{+}\left(z, n, t_{r}\right)\right. \\
&\left.-F_{p}^{+}\left(z, n, t_{r}\right) \widetilde{F}_{r}\left(z, n, t_{r}\right)\right), \\
& R_{2 p+2}(z)=G_{p+1}\left(z, n, t_{r}\right)^{2}-4 a\left(n, t_{r}\right)^{2} F_{p}\left(z, n, t_{r}\right) F_{p}^{+}\left(z, n, t_{r}\right), \\
&\left(z, n, t_{r}\right) \in \mathbb{C} \times \mathbb{Z} \times\left(t_{0, r}-T_{0}, t_{0, r}+T_{0}\right) .
\end{aligned}
$$

Moreover,

$$
\begin{aligned}
& \phi_{t_{r}}\left(P, n, t_{r}\right)=-2 a\left(n, t_{r}\right)\left(\widetilde{F}_{r}\left(z, n, t_{r}\right) \phi\left(P, n, t_{r}\right)^{2}+\widetilde{F}_{r}^{+}\left(z, n, t_{r}\right)\right) \\
&+2\left(z-b^{+}\left(n, t_{r}\right)\right) \widetilde{F}_{r}^{+}\left(z, n, t_{r}\right) \phi\left(P, n, t_{r}\right) \\
&+\left(\widetilde{G}_{r+1}^{+}\left(z, n, t_{r}\right)-\widetilde{G}_{r+1}\left(z, n, t_{r}\right)\right) \phi\left(P, n, t_{r}\right) \\
& a_{t_{r}}\left(n, t_{r}\right)=- a\left(n, t_{r}\right)\left(2\left(z-b^{+}\left(n, t_{r}\right)\right) \widetilde{F}_{r}^{+}\left(z, n, t_{r}\right)\right. \\
&+\left.\widetilde{G}_{r+1}^{+}\left(z, n, t_{r}\right)+\widetilde{G}_{r+1}\left(z, n, t_{r}\right)\right), \\
& b_{t_{r}}\left(n, t_{r}\right)=2\left(\left(z-b\left(n, t_{r}\right)\right)^{2} \widetilde{F}_{r}\left(z, n, t_{r}\right)+\left(z-b\left(n, t_{r}\right)\right) \widetilde{G}_{r+1}\left(z, n, t_{r}\right)\right. \\
&\left.+a\left(n, t_{r}\right)^{2} \widetilde{F}_{r}^{+}\left(z, n, t_{r}\right)-\left(a^{-}\left(n, t_{r}\right)\right)^{2} \widetilde{F}_{r}^{-}\left(z, n, t_{r}\right)\right), \\
&\left(z, n, t_{r}\right) \in \mathbb{C} \times \mathbb{Z} \times\left(t_{0, r}-T_{0}, t_{0, r}+T_{0}\right) .
\end{aligned}
$$


Proof. By Lemma 6.2 we have (5.22), (5.23), (5.27), (5.29)-(5.31), and (6.45)-(6.48) for $\left(n, t_{r}\right) \in \mathbb{Z} \times\left(t_{0, r}-T_{0}, t_{0, r}+T_{0}\right)$ at our disposal. Differentiating (6.52) at $n=n_{0}$ with respect to $t_{r}$, inserting (6.50) and (6.51) at $n=n_{0}$, yields

$$
\begin{aligned}
& 2 F_{p}^{+} a_{t_{r}}+a F_{p, t_{r}}^{+}=2 a\left(G_{p+1} \widetilde{F}_{r}^{+}-F_{p}^{+} \widetilde{G}_{r+1}\right) \\
& \quad=2 F_{p}^{+} a\left(-2\left(z-b^{+}\right) \widetilde{F}_{r}^{+}-\widetilde{G}_{r+1}^{+}-\widetilde{G}_{r+1}\right)+2 a\left(F_{p}^{+} \widetilde{G}_{r+1}^{+}-G_{p+1}^{+} \widetilde{F}_{r}^{+}\right)
\end{aligned}
$$

at $n=n_{0}$. By inspection,

$$
F_{p}^{+}(z) \widetilde{G}_{r+1}^{+}(z)-G_{p+1}^{+}(z) \widetilde{F}_{r}^{+}(z) \underset{|z| \rightarrow \infty}{=} O\left(z^{p-1}\right) .
$$

This can be shown directly using formulas such as (2.23)-(2.26), (6.2), (6.3), (6.5), and (6.6). It also follows from (5.43) and the fact that $F_{p}$ is a monic polynomial of degree $p$. Thus one concludes that

$$
2 F_{p}^{+} a_{t_{r}}=2 F_{p}^{+} a\left(-2\left(z-b^{+}\right) \widetilde{F}_{r}^{+}-\widetilde{G}_{r+1}^{+}-\widetilde{G}_{r+1}\right)
$$

at $n=n_{0}$, and upon cancelling $2 F_{p}^{+}$that (6.54) holds at $n=n_{0}$. This and (6.56) then also prove that (6.50) holds at $n=n_{0}+1$.

Next, differentiating $2 a F_{p} \phi=y-G_{p+1}$ at $n=n_{0}$ with respect to $t_{r}$, inserting (6.50), (6.51), and (6.54) at $n=n_{0}$, and using (5.23) to replace $2 a F_{p}^{+}$ by $-\left(y+G_{p+1}\right) \phi$ and $(5.22)$ to replace $\left(G_{p+1}-y\right)$ by $-2 a F_{p} \phi$, yields $(6.53)$ at $n=n_{0}$ upon cancelling the factor $2 a F_{p}$.

Differentiating (6.46) with respect to $t_{r}$ (fixing $n=n_{0}$ ), inserting (6.46) (to replace $\left.G_{p+1}^{+}\right),(6.51)$ at $n=n_{0}$, and (6.50) at $n=n_{0}+1$ yields

$$
\begin{aligned}
0= & -2 F_{p}^{+}\left(b_{t_{r}}^{+}-2\left(z-b^{+}\right)^{2} \widetilde{F}_{r}^{+}+2 a^{2} \widetilde{F}_{r}-2\left(z-b^{+}\right) \widetilde{G}_{r+1}^{+}\right) \\
& +4\left(z-b^{+}\right)^{2} F_{p}^{+} \widetilde{F}_{r}^{+}+4\left(z-b^{+}\right) G_{p+1} \widetilde{F}_{r}^{+}+4(a)^{2} F_{p} \widetilde{F}_{r}^{+}+G_{p+1, t_{r}}^{+} \\
= & -2 F_{p}^{+}\left(b_{t_{r}}^{+}-2\left(z-b^{+}\right)^{2} \widetilde{F}_{r}^{+}-2\left(z-b^{+}\right) \widetilde{G}_{r+1}^{+}+2 a^{2} \widetilde{F}_{r}-2\left(a^{+}\right)^{2} \widetilde{F}_{r}^{++}\right) \\
& -4\left(a^{+}\right)^{2} F_{p}^{+} \widetilde{F}_{r}^{++}+4\left(z-b^{+}\right)^{2} F_{p}^{+} \widetilde{F}_{r}^{+}+4\left(z-b^{+}\right) G_{p+1} \widetilde{F}_{r}^{+} \\
& +4 a^{2} F_{p} \widetilde{F}_{r}^{+}+G_{p+1, t_{r}}^{+} \\
= & -2 F_{p}^{+}\left(b_{t_{r}}^{+}-2\left(z-b^{+}\right)^{2} \widetilde{F}_{r}^{+}-2\left(z-b^{+}\right) \widetilde{G}_{r+1}^{+}+2 a^{2} \widetilde{F}_{r}-2\left(a^{+}\right)^{2} \widetilde{F}_{r}^{++}\right)
\end{aligned}
$$

$$
\begin{aligned}
& +G_{p+1, t_{r}}^{+}-4\left(a^{+}\right)^{2} F_{p}^{+} \widetilde{F}_{r}^{++} \\
& +\left(4 a^{2} F_{p}+4\left(z-b^{+}\right)^{2} F_{p}^{+}+4\left(z-b^{+}\right) G_{p+1}\right) \widetilde{F}_{r}^{+}
\end{aligned}
$$

at $n=n_{0}$. Combining (6.46) and (6.47) at $n=n_{0}$ one computes

$$
4\left(a^{+}\right)^{2} F_{p}^{++}=4 a^{2} F_{p}+4\left(z-b^{+}\right)^{2} F_{p}^{+}+4\left(z-b^{+}\right) G_{p+1}
$$


at $n=n_{0}$. Insertion of (6.60) into (6.59) then yields

$$
0=-2 F_{p}^{+}\left(b_{t_{r}}^{+}-2\left(z-b^{+}\right)^{2} \widetilde{F}_{r}^{+}-2\left(z-b^{+}\right) \widetilde{G}_{r+1}^{+}+2 a^{2} \widetilde{F}_{r}-2\left(a^{+}\right)^{2} \widetilde{F}_{r}^{++}\right)
$$

$$
+G_{p+1, t_{r}}^{+}-4\left(a^{+}\right)^{2} F_{p}^{+} \widetilde{F}_{r}^{++}+4\left(a^{+}\right)^{2} F_{p}^{++} \widetilde{F}_{r}^{+}
$$

at $n=n_{0}$. In close analogy to (6.57) one observes that

$$
F_{p}^{+}(z) \widetilde{F}_{r}^{++}(z)-F_{p}^{++}(z) \widetilde{F}_{r}^{+}(z) \underset{|z| \rightarrow \infty}{=} O\left(z^{p-1}\right) \text { for } p \in \mathbb{N} .
$$

Thus, since $F_{p}^{+}$is a monic polynomial of degree $p,(6.61)$ proves that

$$
b_{t_{r}}^{+}-2\left(z-b^{+}\right)^{2} \widetilde{F}_{r}^{+}-2\left(z-b^{+}\right) \widetilde{G}_{r+1}^{+}+2 a^{2} \widetilde{F}_{r}-2\left(a^{+}\right)^{2} \widetilde{F}_{r}^{++}=0
$$

at $n=n_{0}$, upon cancelling $F_{p}^{+}$. Thus, (6.55) holds at $n=n_{0}+1$. Simultaneously, this proves (6.51) at $n=n_{0}+1$.

Iterating the arguments just presented (and performing the analogous considerations for $n<n_{0}$ ) then extends these results to all lattice points $n \in \mathbb{Z}$ and hence proves (6.50)-(6.55) for $\left(z, n, t_{r}\right) \in \mathbb{C} \times \mathbb{Z} \times\left(t_{0, r}-T_{0}, t_{0, r}+T_{0}\right)$.

We summarize Lemmas 6.2 and 6.3 next.

Theorem 6.4. Assume Hypothesis 6.1 and condition (6.41). Moreover, suppose that

$$
\begin{aligned}
& f_{j}=f_{j}\left(n_{0}, t_{r}\right), \quad j=1, \ldots, p, \\
& g_{j}=g_{j}\left(n_{0}, t_{r}\right), \quad j=1, \ldots, p-1, \\
& g_{p}+f_{p+1}=g_{p}\left(n_{0}, t_{r}\right)+f_{p+1}\left(t_{r}\right) \\
& \text { for all } t_{r} \in\left(t_{0, r}-T_{0}, t_{0, r}+T_{0}\right),
\end{aligned}
$$

satisfies the autonomous first-order system of ordinary differential equations (6.27) (for fixed $n=n_{0}$ ),

$$
\begin{aligned}
& f_{j, t_{r}}=\mathcal{F}_{j}\left(f_{1}, \ldots, f_{p}, g_{1}, \ldots, g_{p-1}, g_{p}+f_{p+1}\right), \quad j=1, \ldots, p, \\
& g_{j, t_{r}}=\mathcal{G}_{j}\left(f_{1}, \ldots, f_{p}, g_{1}, \ldots, g_{p-1}, g_{p}+f_{p+1}\right), \quad j=1, \ldots, p-1, \\
& \left(g_{p}+f_{p+1}\right)_{t_{r}}=\mathcal{G}_{p}\left(f_{1}, \ldots, f_{p}, g_{1}, \ldots, g_{p-1}, g_{p}+f_{p+1}\right)
\end{aligned}
$$

with initial condition

$$
\begin{aligned}
& f_{j}\left(n_{0}, t_{0, r}\right), \quad j=1, \ldots, p, \\
& g_{j}\left(n_{0}, t_{0, r}\right), \quad j=1, \ldots, p-1, \\
& g_{p}\left(n_{0}, t_{0, r}\right)+f_{p+1}\left(t_{0, r}\right) .
\end{aligned}
$$


Then $F_{p}$ and $G_{p+1}$ as constructed in (6.2)-(6.44) on $\mathbb{C} \times \mathbb{Z} \times\left(t_{0, r}-T_{0}, t_{0, r}+T_{0}\right)$ satisfy the zero-curvature equations (5.8), (5.9), and (5.45),

$$
\begin{array}{r}
U_{t_{r}}+U \widetilde{V}_{r+1}-\widetilde{V}_{r+1}^{+} U=0, \\
U V_{p+1}-V_{p+1}^{+} U=0, \\
V_{p+1, t_{r}}-\left[\widetilde{V}_{r+1}, V_{p+1}\right]=0 \\
\text { on } \mathbb{C} \times \mathbb{Z} \times\left(t_{0, r}-T_{0}, t_{0, r}+T_{0}\right),
\end{array}
$$

with $U, V_{p+1}$, and $\widetilde{V}_{r+1}$ given by (5.10). In particular, a, b satisfy the algebrogeometric initial value problem (5.2), (5.3) on $\mathbb{Z} \times\left(t_{0, r}-T_{0}, t_{0, r}+T_{0}\right)$,

$$
\begin{aligned}
& \widetilde{\mathrm{Tl}}_{r}(a, b)=\left(\begin{array}{c}
a_{t_{r}}-a\left(\tilde{f}_{p+1}^{+}(a, b)-\tilde{f}_{p+1}(a, b)\right) \\
b_{t_{r}}+\tilde{g}_{p+1}(a, b)-\tilde{g}_{p+1}^{-}(a, b)
\end{array}\right)=0, \\
& \left.(a, b)\right|_{t_{r}=t_{0, r}}=\left(a^{(0)}, b^{(0)}\right), \\
& \mathrm{S}^{-} \mathrm{Tl}_{p}\left(a^{(0)}, b^{(0)}\right)=\left(\begin{array}{c}
-a\left(f_{p+1}^{+}\left(p^{(0)}, q^{(0)}\right)-f_{p+1}\left(p^{(0)}, q^{(0)}\right)\right) \\
g_{p+1}\left(a^{(0)}, b^{(0)}\right)-g_{p+1}^{-}\left(a^{(0)}, b^{(0)}\right)
\end{array}\right)=0,
\end{aligned}
$$

and are given by

$$
\begin{aligned}
a\left(n, t_{r}\right)^{2}= & \frac{1}{2} \sum_{k=1}^{q\left(n, t_{r}\right)} \frac{\left.\left(d^{\ell}\left(R_{2 p+2}(z)^{1 / 2}\right) / d z^{\ell}\right)\right|_{z=\mu_{k}\left(n, t_{r}\right)}}{\left(p_{k}\left(n, t_{r}\right)-1\right) !} \\
& \times \prod_{k^{\prime}=1, k^{\prime} \neq k}^{q\left(n, t_{r}\right)}\left(\mu_{k}\left(n, t_{r}\right)-\mu_{k^{\prime}}\left(n, t_{r}\right)\right)^{-p_{k}\left(n, t_{r}\right)} \\
& +\frac{1}{4}\left(b^{(2)}\left(n, t_{r}\right)-b\left(n, t_{r}\right)^{2}\right), \\
b\left(n, t_{r}\right)= & \frac{1}{2} \sum_{m=0}^{2 p+1} E_{m}-\sum_{k=1}^{q\left(n, t_{r}\right)} p_{k}\left(n, t_{r}\right) \mu_{k}\left(n, t_{r}\right), \\
& \left(z, n, t_{r}\right) \in \mathbb{Z} \times\left(t_{0, r}-T_{0}, t_{0, r}+T_{0}\right) .
\end{aligned}
$$

Moreover, Lemmas 3.2-3.4 and 5.2-5.6 apply.

As in the stationary case, the theta function representations of $a$ and $b$ in the time-dependent context can be derived in complete analogy to the self-adjoint case. Since the final results are formally the same as in the selfadjoint case we again just refer, for instance, to [6], [7], [9], [10], [14, Sect. 1.4], [18] (cf. also the appendix written in [8]), [25], [30, Appendix, Sect. 9], [32, Sect. 13.2], [33, Sect. 4.6], [34, Ch. 28].

As in Lemma 4.2 we now show that also in the time-dependent case, most initial divisors are nice in the sense that the corresponding divisor trajectory stays away from $P_{\infty_{ \pm}}$for all $\left(n, t_{r}\right) \in \mathbb{Z} \times \mathbb{R}$. 
Lemma 6.5. The set $\mathcal{M}_{1}$ of initial divisors $\mathcal{D}_{\underline{\hat{\mu}}\left(n_{0}, t_{0, r}\right)}$ for which $\mathcal{D}_{\underline{\hat{\mu}}\left(n, t_{r}\right)}$, defined via (5.53), is nonspecial and finite for all $\left(n, t_{r}\right) \in \mathbb{Z} \times \mathbb{R}$, forms a dense set of full measure in the set $\operatorname{Sym}^{p}\left(\mathcal{K}_{p}\right)$ of nonnegative divisors of degree $p$.

Proof. Let $\mathcal{M}_{\text {sing }}$ be as introduced in the proof of Lemma 4.2. Then

$$
\begin{aligned}
\bigcup_{t_{r} \in \mathbb{R}}\left(\underline{\alpha}_{Q_{0}}\left(\mathcal{M}_{\text {sing }}\right)+t_{r} \underline{\widetilde{U}}_{r}^{(2)}\right) \\
=\bigcup_{t_{r} \in \mathbb{R}}\left(\underline{A}_{Q_{0}}\left(P_{\infty_{+}}\right)+\underline{\alpha}_{Q_{0}}\left(\operatorname{Sym}^{p-1}\left(\mathcal{K}_{p}\right)\right)+t_{r} \underline{\widetilde{U}}_{r}^{(2)}\right) \\
\cup \bigcup_{t_{r} \in \mathbb{R}}\left(\underline{A}_{Q_{0}}\left(P_{\infty_{-}}\right)+\underline{\alpha}_{Q_{0}}\left(\operatorname{Sym}^{p-1}\left(\mathcal{K}_{p}\right)\right)+t_{r} \underline{\widetilde{U}}_{r}^{(2)}\right)
\end{aligned}
$$

is of measure zero as well, since it is contained in the image of $\mathbb{R} \times \operatorname{Sym}^{p-1}\left(\mathcal{K}_{p}\right)$ which misses one real dimension in comparison to the $2 p$ real dimensions of $J\left(\mathcal{K}_{p}\right)$. But then

$$
\bigcup_{\left(n, t_{r}\right) \in \mathbb{Z} \times \mathbb{R}}\left(\underline{\alpha}_{Q_{0}}\left(\mathcal{M}_{\text {sing }}\right)+n \underline{A}_{P_{\infty_{-}}}\left(P_{\infty_{+}}\right)+t_{r} \underline{\widetilde{U}}_{r}^{(2)}\right)
$$

is also of measure zero. Applying $\underline{\alpha}_{Q_{0}}^{-1}$ to the complement of the set in (6.75) then yields a set $\mathcal{M}_{1}$ of full measure in $\operatorname{Sym}^{p}\left(\mathcal{K}_{p}\right)$. In particular, $\mathcal{M}_{1}$ is necessarily dense in $\operatorname{Sym}^{p}\left(\mathcal{K}_{p}\right)$.

Theorem 6.6. Let $\mathcal{D}_{\hat{\mu}\left(n_{0}, t_{0, r}\right)} \in \mathcal{M}_{1}$ be an initial divisor as in Lemma 6.5. Then the sequences $a, \bar{b}$ constructed from $\hat{\mu}\left(n_{0}, t_{0, r}\right)$ as described in Theorem 6.4 satisfy Hypothesis 5.1. In particular, the solution a, $b$ of the algebrogeometric initial value problem (6.70), (6.71) is global in $\left(n, t_{r}\right) \in \mathbb{Z} \times \mathbb{R}$.

Proof. Starting with $\mathcal{D}_{\underline{\hat{\mu}}\left(n_{0}, t_{0, r}\right)} \in \mathcal{M}_{1}$, the procedure outlined in this section and summarized in Theorem 6.4 leads to $\mathcal{D}_{\underline{\hat{\mu}}\left(n, t_{r}\right)}$ for all $\left(n, t_{r}\right) \in$ $\mathbb{Z} \times\left(t_{0, r}-T_{0}, t_{0, r}+T_{0}\right)$ such that (5.53)holds. But if $a, b$ should blow up, then $\mathcal{D}_{\hat{\mu}\left(n, t_{r}\right)}$ must hit $P_{\infty_{+}}$or $P_{\infty_{-}}$which is impossible by our choice of initial condition.

Note, however, that in general (i.e., unless one is, e.g., in the special periodic or self-adjoint case), $\mathcal{D}_{\underline{\hat{\mu}}\left(n, t_{r}\right)}$ will get arbitrarily close to $P_{\infty_{+}}$since straight motions on the torus are generically dense (see e.g. [2, Sect. 51] or $[17$, Sects. $1.4,1.5])$ and hence no uniform bound on the sequences $a\left(n, t_{r}\right), b\left(n, t_{r}\right)$ exists as $\left(n, t_{r}\right)$ vary in $\mathbb{Z} \times \mathbb{R}$. In particular, these complexvalued algebro-geometric solutions of the Toda hierarchy initial value problem, in general, will not be quasi-periodic (cf. the usual definition of quasiperiodic functions, e.g., in [31, p. 31]) with respect to $n$ or $t_{r}$. 


\section{A. Hyperelliptic curves of the Toda-type}

We provide a brief summary of some of the fundamental notations needed from the theory of hyperelliptic Riemann surfaces. More details can be found in some of the standard textbooks [11] and [27] as well as in monographs and surveys dedicated to integrable systems such as [5, Ch. 2], [8], [13, App. A, B], [32, App. A].

Fix $p \in \mathbb{N}$. We intend to describe the hyperelliptic Riemann surface $\mathcal{K}_{p}$ of genus $p$ of the Toda-type curve (2.43), associated with the polynomial

$$
\begin{aligned}
& \mathcal{F}_{p}(z, y)=y^{2}-R_{2 p+2}(z)=0 \\
& R_{2 p+2}(z)=\prod_{m=0}^{2 p+1}\left(z-E_{m}\right), \quad\left\{E_{m}\right\}_{m=0}^{2 p+1} \subset \mathbb{C} .
\end{aligned}
$$

To simplify the discussion we will assume that the affine part of $\mathcal{K}_{p}$ is nonsingular, that is, we assume that

$$
E_{m} \neq E_{m^{\prime}} \text { for } m \neq m^{\prime}, m, m^{\prime}=0, \ldots, 2 p+1
$$

throughout this appendix. Next we introduce an appropriate set of (nonintersecting) cuts $\mathcal{C}_{j}$ joining $E_{m(j)}$ and $E_{m^{\prime}(j)}, j=1, \ldots, p+1$, and denote

$$
\mathcal{C}=\bigcup_{j=1}^{p+1} \mathcal{C}_{j}, \quad \mathcal{C}_{j} \cap \mathcal{C}_{k}=\emptyset, \quad j \neq k
$$

Define the cut plane

$$
\Pi=\mathbb{C} \backslash \mathcal{C}
$$

and introduce the holomorphic function

$$
R_{2 p+2}(\cdot)^{1 / 2}: \Pi \rightarrow \mathbb{C}, \quad z \mapsto\left(\prod_{m=0}^{2 p+1}\left(z-E_{m}\right)\right)^{1 / 2}
$$

on $\Pi$ with an appropriate choice of the square root branch in (A.5). Next we define the set

$$
\mathcal{M}_{p}=\left\{\left(z, \sigma R_{2 p+2}(z)^{1 / 2}\right) \mid z \in \mathbb{C}, \sigma \in\{1,-1\}\right\} \cup\left\{P_{\infty_{+}}, P_{\infty_{-}}\right\}
$$

by extending $R_{2 p+2}(\cdot)^{1 / 2}$ to $\mathcal{C}$. The hyperelliptic curve $\mathcal{K}_{p}$ is then the set $\mathcal{M}_{p}$ with its natural complex structure obtained upon gluing the two sheets 
of $\mathcal{M}_{p}$ crosswise along the cuts. Moreover, we introduce the set of branch points

$$
\mathcal{B}\left(\mathcal{K}_{p}\right)=\left\{\left(E_{m}, 0\right)\right\}_{m=0}^{2 p+1}
$$

Points $P \in \mathcal{K}_{p} \backslash\left\{P_{\infty_{ \pm}}\right\}$are denoted by

$$
P=\left(z, \sigma R_{2 p+2}(z)^{1 / 2}\right)=(z, y),
$$

where $y$ denotes the meromorphic function on $\mathcal{K}_{p}$ satisfying $\mathcal{F}_{p}(z, y)=y^{2}-$ $R_{2 p+2}(z)=0$ and

(A.9) $y(P) \underset{\zeta \rightarrow 0}{=} \mp\left(1-\frac{1}{2}\left(\sum_{m=0}^{2 p+1} E_{m}\right) \zeta+O\left(\zeta^{2}\right)\right) \zeta^{-p-1}$ as $P \rightarrow P_{\infty_{ \pm}}, \zeta=1 / z$.

In addition, we introduce the holomorphic sheet exchange map (involution)

$$
(\mathrm{A} .10) \quad *: \mathcal{K}_{p} \rightarrow \mathcal{K}_{p}, \quad P=(z, y) \mapsto P^{*}=(z,-y), P_{\infty_{ \pm}} \mapsto P_{\infty_{ \pm}}^{*}=P_{\infty_{\mp}}
$$

and the two meromorphic projection maps

$$
\tilde{\pi}: \mathcal{K}_{p} \rightarrow \mathbb{C} \cup\{\infty\}, \quad P=(z, y) \mapsto z, P_{\infty_{ \pm}} \mapsto \infty
$$

and

$$
y: \mathcal{K}_{p} \rightarrow \mathbb{C} \cup\{\infty\}, \quad P=(z, y) \mapsto y, P_{\infty_{ \pm}} \mapsto \infty .
$$

Thus the map $\tilde{\pi}$ has a pole of order 1 at $P_{\infty_{ \pm}}$and $y$ has a pole of order $p+1$ at $P_{\infty_{ \pm}}$. Moreover,

$$
\tilde{\pi}\left(P^{*}\right)=\tilde{\pi}(P), \quad y\left(P^{*}\right)=-y(P), \quad P \in \mathcal{K}_{p} .
$$

As a result, $\mathcal{K}_{p}$ is a two-sheeted branched covering of the Riemann sphere $\mathbb{C P}^{1}(\cong \mathbb{C} \cup\{\infty\})$ branched at the $2 p+4$ points $\left\{\left(E_{m}, 0\right)\right\}_{m=0}^{2 p+1}, P_{\infty_{ \pm}} . \mathcal{K}_{p}$ is compact since $\tilde{\pi}$ is open and $\mathbb{C P}^{1}$ is compact. Therefore, the compact hyperelliptic Riemann surface resulting in this manner has topological genus $p$.

Next we introduce the upper and lower sheets $\Pi_{ \pm}$by

$$
\Pi_{ \pm}=\left\{\left(z, \pm R_{2 p+2}(z)^{1 / 2}\right) \in \mathcal{M}_{p} \mid z \in \Pi\right\}
$$

and the associated charts

$$
\zeta_{ \pm}: \Pi_{ \pm} \rightarrow \Pi, \quad P \mapsto z
$$


Let $\left\{a_{j}, b_{j}\right\}_{j=1}^{p}$ be a homology basis for $\mathcal{K}_{p}$ with intersection matrix of the cycles satisfying

$$
a_{j} \circ b_{k}=\delta_{j, k}, \quad a_{j} \circ a_{k}=0, \quad b_{j} \circ b_{k}=0, \quad j, k=1, \ldots, p .
$$

Associated with the homology basis $\left\{a_{j}, b_{j}\right\}_{j=1}^{p}$ we also recall the canonical dissection of $\mathcal{K}_{p}$ along its cycles yielding the simply connected interior $\widehat{\mathcal{K}}_{p}$ of the fundamental polygon $\partial \widehat{\mathcal{K}}_{p}$ given by

$$
\partial \widehat{\mathcal{K}}_{p}=a_{1} b_{1} a_{1}^{-1} b_{1}^{-1} a_{2} b_{2} a_{2}^{-1} b_{2}^{-1} \cdots a_{p}^{-1} b_{p}^{-1} .
$$

Let $\mathcal{M}\left(\mathcal{K}_{n}\right)$ and $\mathcal{M}^{1}\left(\mathcal{K}_{n}\right)$ denote the set of meromorphic functions (0forms) and meromorphic differentials (1-forms) on $\mathcal{K}_{n}$. The residue of a meromorphic differential $\nu \in \mathcal{M}^{1}\left(\mathcal{K}_{n}\right)$ at a point $Q \in \mathcal{K}_{n}$ is defined by

$$
\operatorname{res}_{Q}(\nu)=\frac{1}{2 \pi i} \int_{\gamma_{Q}} \nu
$$

where $\gamma_{Q}$ is a counterclockwise oriented smooth simple closed contour encircling $Q$ but no other pole of $\nu$. Holomorphic differentials are also called Abelian differentials of the first kind. Abelian differentials of the second kind $\omega^{(2)} \in \mathcal{M}^{1}\left(\mathcal{K}_{n}\right)$ are characterized by the property that all their residues vanish. They will usually be normalized by demanding that all their $a$-periods vanish, that is,

$$
\int_{a_{j}} \omega^{(2)}=0, \quad j=1, \ldots, p
$$

If $\omega_{P_{1}, n}^{(2)}$ is a differential of the second kind on $\mathcal{K}_{n}$ whose only pole is $P_{1} \in \widehat{\mathcal{K}}_{n}$ with principal part $\zeta^{-n-2} d \zeta, n \in \mathbb{N}_{0}$ near $P_{1}$ and $\omega_{j}=\left(\sum_{m=0}^{\infty} d_{j, m}\left(P_{1}\right) \zeta^{m}\right) d \zeta$ near $P_{1}$, then

$$
\frac{1}{2 \pi i} \int_{b_{j}} \omega_{P_{1}, m}^{(2)}=\frac{d_{j, m}\left(P_{1}\right)}{m+1}, \quad m=0,1, \ldots
$$

Using local charts one infers that $d z / y$ is a holomorphic differential on $\mathcal{K}_{p}$ with zeros of order $p-1$ at $P_{\infty_{ \pm}}$and hence

$$
\eta_{j}=\frac{z^{j-1} d z}{y}, \quad j=1, \ldots, p,
$$

form a basis for the space of holomorphic differentials on $\mathcal{K}_{p}$. Introducing the invertible matrix $C$ in $\mathbb{C}^{p}$

$$
\begin{aligned}
C & =\left(C_{j, k}\right)_{j, k=1, \ldots, p}, \quad C_{j, k}=\int_{a_{k}} \eta_{j}, \\
\underline{c}(k) & =\left(c_{1}(k), \ldots, c_{p}(k)\right), \quad c_{j}(k)=\left(C^{-1}\right)_{j, k}, \quad j, k=1, \ldots, p,
\end{aligned}
$$


the normalized differentials $\omega_{j}$ for $j=1, \ldots, p$,

$$
\omega_{j}=\sum_{\ell=1}^{p} c_{j}(\ell) \eta_{\ell}, \quad \int_{a_{k}} \omega_{j}=\delta_{j, k}, \quad j, k=1, \ldots, p,
$$

form a canonical basis for the space of holomorphic differentials on $\mathcal{K}_{p}$.

In the chart $\left(U_{P_{\infty_{ \pm}}}, \zeta_{P_{\infty_{ \pm}}}\right)$induced by $1 / \tilde{\pi}$ near $P_{\infty_{ \pm}}$one infers,

$$
\begin{aligned}
& \underline{\omega}=\left(\omega_{1}, \ldots, \omega_{p}\right)=\mp \sum_{j=1}^{p} \frac{\underline{c}(j) \zeta^{p-j} d \zeta}{\left(\prod_{m=0}^{2 p+1}\left(1-\zeta E_{m}\right)\right)^{1 / 2}} \\
&= \pm\left(\underline{c}(p)+\zeta\left(\frac{1}{2} \underline{c}(p) \sum_{m=0}^{2 p+1} E_{m}+\underline{c}(p-1)\right)+O\left(\zeta^{2}\right)\right) d \zeta \text { as } P \rightarrow P_{\infty_{ \pm}}, \\
& \zeta=1 / z .
\end{aligned}
$$

The matrix $\tau=\left(\tau_{j, \ell}\right)_{j, \ell=1}^{p}$ in $\mathbb{C}^{p \times p}$ of $b$-periods defined by

$$
\tau_{j, \ell}=\int_{b_{j}} \omega_{\ell}, \quad j, \ell=1, \ldots, p,
$$

satisfies

$$
\operatorname{Im}(\tau)>0 \text { and } \tau_{j, \ell}=\tau_{\ell, j}, j, \ell=1, \ldots, p .
$$

Associated with the matrix $\tau$ one introduces the period lattice

$$
L_{p}=\left\{\underline{z} \in \mathbb{C}^{p} \mid \underline{z}=\underline{m}+\underline{n} \tau, \underline{m}, \underline{n} \in \mathbb{Z}^{p}\right\}
$$

and the Riemann theta function associated with $\mathcal{K}_{n}$ and the given homology basis $\left\{a_{j}, b_{j}\right\}_{j=1, \ldots, n}$,

$$
\theta(\underline{z})=\sum_{\underline{n} \in \mathbb{Z}^{n}} \exp (2 \pi i(\underline{n}, \underline{z})+\pi i(\underline{n}, \underline{n} \tau)), \quad \underline{z} \in \mathbb{C}^{n},
$$

where $(\underline{u}, \underline{v})=\underline{\bar{u}} \underline{v}^{\top}=\sum_{j=1}^{n} \overline{u_{j}} v_{j}$ denotes the scalar product in $\mathbb{C}^{n}$. It has the fundamental properties

(A.30) $\theta\left(z_{1}, \ldots, z_{j-1},-z_{j}, z_{j+1}, \ldots, z_{n}\right)=\theta(\underline{z})$,

(A.31) $\theta(\underline{z}+\underline{m}+\underline{n} \tau)=\exp (-2 \pi i(\underline{n}, \underline{z})-\pi i(\underline{n}, \underline{n} \tau)) \theta(\underline{z}), \quad \underline{m}, \underline{n} \in \mathbb{Z}^{n}$.

Next, fixing a base point $Q_{0} \in \mathcal{K}_{p} \backslash\left\{P_{\infty_{ \pm}}\right\}$, one denotes by $J\left(\mathcal{K}_{p}\right)=$ $\mathbb{C}^{p} / L_{p}$ the Jacobi variety of $\mathcal{K}_{p}$, and defines the Abel map $\underline{A}_{Q_{0}}$ by

$$
\begin{gathered}
\underline{A}_{Q_{0}}: \mathcal{K}_{n} \rightarrow J\left(\mathcal{K}_{p}\right), \quad \underline{A}_{Q_{0}}(P)=\left(\int_{Q_{0}}^{P} \omega_{1}, \ldots, \int_{Q_{0}}^{P} \omega_{p}\right) \quad\left(\bmod L_{p}\right), \\
P \in \mathcal{K}_{p} .
\end{gathered}
$$


Similarly, one introduces

$$
\underline{\alpha}_{Q_{0}}: \operatorname{Div}\left(\mathcal{K}_{p}\right) \rightarrow J\left(\mathcal{K}_{p}\right), \quad \mathcal{D} \mapsto \underline{\alpha}_{Q_{0}}(\mathcal{D})=\sum_{P \in \mathcal{K}_{p}} \mathcal{D}(P) \underline{A}_{Q_{0}}(P),
$$

where $\operatorname{Div}\left(\mathcal{K}_{p}\right)$ denotes the set of divisors on $\mathcal{K}_{p}$. Here a map $\mathcal{D}: \mathcal{K}_{p} \rightarrow \mathbb{Z}$ is called a divisor on $\mathcal{K}_{p}$ if $\mathcal{D}(P) \neq 0$ for only finitely many $P \in \mathcal{K}_{p}$. (In the main body of this paper we will choose $Q_{0}$ to be one of the branch points, i.e., $Q_{0} \in \mathcal{B}\left(\mathcal{K}_{p}\right)$, and for simplicity we will always choose the same path of integration from $Q_{0}$ to $P$ in all Abelian integrals.)

In connection with divisors on $\mathcal{K}_{p}$ we will employ the following (additive) notation,

$$
\begin{aligned}
& \mathcal{D}_{Q_{0} \underline{Q}}=\mathcal{D}_{Q_{0}}+\mathcal{D}_{\underline{Q}}, \quad \mathcal{D}_{\underline{Q}}=\mathcal{D}_{Q_{1}}+\cdots+\mathcal{D}_{Q_{m}}, \\
& \underline{Q}=\left\{Q_{1}, \ldots, Q_{m}\right\} \in \operatorname{Sym}^{m} \mathcal{K}_{p}, \quad Q_{0} \in \mathcal{K}_{p}, \quad m \in \mathbb{N},
\end{aligned}
$$

where for any $Q \in \mathcal{K}_{p}$,

$$
\mathcal{D}_{Q}: \mathcal{K}_{p} \rightarrow \mathbb{N}_{0}, \quad P \mapsto \mathcal{D}_{Q}(P)= \begin{cases}1 & \text { for } P=Q, \\ 0 & \text { for } P \in \mathcal{K}_{p} \backslash\{Q\},\end{cases}
$$

and $\operatorname{Sym}^{m} \mathcal{K}_{p}$ denotes the $m$ th symmetric product of $\mathcal{K}_{p}$. In particular, $\operatorname{Sym}^{m} \mathcal{K}_{p}$ can be identified with the set of nonnegative divisors $0 \leq \mathcal{D} \in$ $\operatorname{Div}\left(\mathcal{K}_{p}\right)$ of degree $m \in \mathbb{N}$. A divisor $\mathcal{D}_{\underline{Q}}=\mathcal{D}_{Q_{1}}+\cdots+\mathcal{D}_{Q_{m}}$ will be called finite if $Q_{k} \in \mathcal{K}_{p} \backslash\left\{P_{\infty_{+}}, P_{\infty_{-}}\right\}, k=1, \ldots, m$.

For $f \in \mathcal{M}\left(\mathcal{K}_{p}\right) \backslash\{0\}, \omega \in \mathcal{M}^{1}\left(\mathcal{K}_{p}\right) \backslash\{0\}$ the divisors of $f$ and $\omega$ are denoted by $(f)$ and $(\omega)$, respectively. Two divisors $\mathcal{D}, \mathcal{E} \in \operatorname{Div}\left(\mathcal{K}_{p}\right)$ are called equivalent, denoted by $\mathcal{D} \sim \mathcal{E}$, if and only if $\mathcal{D}-\mathcal{E}=(f)$ for some $f \in \mathcal{M}\left(\mathcal{K}_{p}\right) \backslash\{0\}$. The divisor class $[\mathcal{D}]$ of $\mathcal{D}$ is then given by $[\mathcal{D}]=\{\mathcal{E} \in$ $\left.\operatorname{Div}\left(\mathcal{K}_{p}\right) \mid \mathcal{E} \sim \mathcal{D}\right\}$. We recall that

$$
\begin{aligned}
& \operatorname{deg}((f))=0, \quad \operatorname{deg}((\omega))=2(p-1), \\
& f \in \mathcal{M}\left(\mathcal{K}_{p}\right) \backslash\{0\}, \omega \in \mathcal{M}^{1}\left(\mathcal{K}_{p}\right) \backslash\{0\}
\end{aligned}
$$

where the degree $\operatorname{deg}(\mathcal{D})$ of $\mathcal{D}$ is given by $\operatorname{deg}(\mathcal{D})=\sum_{P \in \mathcal{K}_{p}} \mathcal{D}(P)$. It is customary to call $(f)$ (respectively, $(\omega)$ ) a principal (respectively, canonical) divisor.

Introducing the complex linear spaces

$(\mathrm{A} .37) \quad \mathcal{L}(\mathcal{D})=\left\{f \in \mathcal{M}\left(\mathcal{K}_{p}\right) \mid f=0\right.$ or $\left.(f) \geq \mathcal{D}\right\}, \quad r(\mathcal{D})=\operatorname{dim}_{\mathbb{C}} \mathcal{L}(\mathcal{D})$, $(\mathrm{A} .38) \mathcal{L}^{1}(\mathcal{D})=\left\{\omega \in \mathcal{M}^{1}\left(\mathcal{K}_{p}\right) \mid \omega=0\right.$ or $\left.(\omega) \geq \mathcal{D}\right\}, \quad i(\mathcal{D})=\operatorname{dim}_{\mathbb{C}} \mathcal{L}^{1}(\mathcal{D})$

(with $i(\mathcal{D})$ the index of specialty of $\mathcal{D}$ ), one infers that $\operatorname{deg}(\mathcal{D}), r(\mathcal{D})$, and $i(\mathcal{D})$ only depend on the divisor class $[\mathcal{D}]$ of $\mathcal{D}$. Moreover, we recall the following fundamental facts. 
Theorem A.1. Let $\mathcal{D} \in \operatorname{Div}\left(\mathcal{K}_{p}\right), \omega \in \mathcal{M}^{1}\left(\mathcal{K}_{p}\right) \backslash\{0\}$. Then,

$$
i(\mathcal{D})=r(\mathcal{D}-(\omega)), \quad p \in \mathbb{N}_{0} .
$$

The Riemann-Roch theorem reads

$$
r(-\mathcal{D})=\operatorname{deg}(\mathcal{D})+i(\mathcal{D})-p+1, \quad n \in \mathbb{N}_{0} .
$$

By Abel's theorem, $\mathcal{D} \in \operatorname{Div}\left(\mathcal{K}_{p}\right), p \in \mathbb{N}$, is principal if and only if

$$
\operatorname{deg}(\mathcal{D})=0 \text { and } \underline{\alpha}_{Q_{0}}(\mathcal{D})=\underline{0} \text {. }
$$

Finally, assume $p \in \mathbb{N}$. Then $\underline{\alpha}_{Q_{0}}: \operatorname{Div}\left(\mathcal{K}_{p}\right) \rightarrow J\left(\mathcal{K}_{p}\right)$ is surjective (Jacobi's inversion theorem).

Theorem A.2. Let $\mathcal{D}_{\underline{Q}} \in \operatorname{Sym}^{p} \mathcal{K}_{p}, \underline{Q}=\left\{Q_{1}, \ldots, Q_{p}\right\}$. Then,

$$
1 \leq i\left(\mathcal{D}_{\underline{Q}}\right)=s
$$

if and only if there are $s$ pairs of the type $\left\{P, P^{*}\right\} \subseteq\left\{Q_{1}, \ldots, Q_{p}\right\}$ (this includes, of course, branch points for which $\left.P=P^{*}\right)$. Obviously, one has $s \leq p / 2$.

Next, we denote by $\Xi_{Q_{0}}=\left(\Xi_{Q_{0,1}}, \ldots, \Xi_{Q_{0, p}}\right)$ the vector of Riemann constants,

$$
\Xi_{Q_{0, j}}=\frac{1}{2}\left(1+\tau_{j, j}\right)-\sum_{\substack{\ell=1 \\ \ell \neq j}}^{p} \int_{a_{\ell}} \omega_{\ell}(P) \int_{Q_{0}}^{P} \omega_{j}, \quad j=1, \ldots, p .
$$

Theorem A.3. Let $\underline{Q}=\left\{Q_{1}, \ldots, Q_{p}\right\} \in \operatorname{Sym}^{p} \mathcal{K}_{p}$ and assume $\mathcal{D}_{\underline{Q}}$ to be nonspecial, that is, $i\left(\overline{\mathcal{D}}_{\underline{Q}}\right)=0$. Then,

(A.44) $\theta\left(\Xi_{Q_{0}}-\underline{A}_{Q_{0}}(P)+\alpha_{Q_{0}}\left(\mathcal{D}_{\underline{Q}}\right)\right)=0$ if and only if $P \in\left\{Q_{1}, \ldots, Q_{p}\right\}$.

\section{B. Some interpolation formulas}

In this appendix we recall a useful interpolation formula which goes beyond the standard Lagrange interpolation formula for polynomials in the sense that the zeros of the interpolating polynomial need not be distinct.

Lemma B.1. Let $p \in \mathbb{N}$ and $S_{p-1}$ be a polynomial of degree $p-1$. In addition, let $F_{p}$ be a monic polynomial of degree $p$ of the form

$$
F_{p}(z)=\prod_{k=1}^{q}\left(z-\mu_{k}\right)^{p_{k}}, \quad p_{j} \in \mathbb{N}, \mu_{j} \in \mathbb{C}, j=1, \ldots, q, \quad \sum_{k=1}^{q} p_{k}=p .
$$


Then,

$$
\begin{aligned}
& S_{p-1}(z)=F_{p}(z) \sum_{k=1}^{q} \sum_{\ell=0}^{p_{k}-1} \frac{S_{p-1}^{(\ell)}\left(\mu_{k}\right)}{\ell !\left(p_{k}-\ell-1\right) !} \\
& \quad \times\left.\left(\frac{d^{p_{k}-\ell-1}}{d \zeta^{p_{k}-\ell-1}}\left((z-\zeta)^{-1} \prod_{k^{\prime}=1, k^{\prime} \neq k}^{q}\left(\zeta-\mu_{k^{\prime}}\right)^{-p_{k^{\prime}}}\right)\right)\right|_{\zeta=\mu_{k}}, \quad z \in \mathbb{C} .
\end{aligned}
$$

In particular, $S_{p-1}$ is uniquely determined by prescribing the $p$ values

$$
S_{p-1}\left(\mu_{k}\right), S_{p-1}^{\prime}\left(\mu_{k}\right), \ldots, S_{p-1}^{\left(p_{k}-1\right)}\left(\mu_{k}\right), \quad k=1, \ldots, q,
$$

at the given points $\mu_{1} \ldots, \mu_{q}$.

Conversely, prescribing the $p$ complex numbers

$$
\alpha_{k}^{(0)}, \alpha_{k}^{(1)}, \ldots, \alpha_{k}^{\left(p_{k}-1\right)}, \quad k=1, \ldots, q,
$$

there exists a unique polynomial $T_{p-1}$ of degree $p-1$,

$$
\begin{aligned}
& T_{p-1}(z)=F_{p}(z) \sum_{k=1}^{q} \sum_{\ell=0}^{p_{k}-1} \frac{\alpha_{k}^{(\ell)}}{\ell !\left(p_{k}-\ell-1\right) !} \\
& \quad \times\left.\left(\frac{d^{p_{k}-\ell-1}}{d \zeta^{p_{k}-\ell-1}}\left((z-\zeta)^{-1} \prod_{k^{\prime}=1, k^{\prime} \neq k}^{q}\left(\zeta-\mu_{k^{\prime}}\right)^{-p_{k^{\prime}}}\right)\right)\right|_{\zeta=\mu_{k}}, \quad z \in \mathbb{C},
\end{aligned}
$$

such that

$$
\begin{array}{r}
T_{p-1}\left(\mu_{k}\right)=\alpha_{k}^{(0)}, T_{p-1}^{\prime}\left(\mu_{k}\right)=\alpha_{k}^{(1)}, \ldots, T_{p-1}^{\left(p_{k}-1\right)}\left(\mu_{k}\right)=\alpha_{k}^{\left(p_{k}-1\right)}, \\
k=1, \ldots, q .
\end{array}
$$

Proof. Our starting point for proving (B.2) is the following formula derived, for instance, by Markushevich [22, Part 2, Sect. 2.11, p. 68],

$$
S_{p-1}(z)=\frac{1}{2 \pi i} \oint_{\Gamma} \frac{d \zeta S_{p-1}(\zeta)}{F_{p}(\zeta)} \frac{F_{p}(\zeta)-F_{p}(z)}{\zeta-z}, \quad z \in \mathbb{C},
$$

where $\Gamma$ is a simple, smooth, counterclockwise oriented curve encircling $\mu_{1}, \ldots, \mu_{q}$. Since the integrand in (B.7) is analytic at the point $\zeta=z$, we may, without loss of generality, assume that $\Gamma$ does not encircle $z$. With this assumption one obtains

$$
\frac{1}{2 \pi i} \oint_{\Gamma} \frac{d \zeta S_{p-1}(\zeta)}{\zeta-z}=0
$$

and hence deforming $\Gamma$ into sufficiently small counterclockwise oriented circles $\Gamma_{k}$ with center at $\mu_{k}, k=1, \ldots, q$, such that no $\mu_{k^{\prime}}, k^{\prime} \neq k$, is encircled 
by $\Gamma_{k}$, one obtains

$$
\begin{aligned}
S_{p-1}(z) & =-\frac{F_{p}(z)}{2 \pi i} \oint_{\Gamma} \frac{d \zeta S_{p-1}(\zeta)}{F_{p}(\zeta)(\zeta-z)} \\
& =-\frac{F_{p}(z)}{2 \pi i} \sum_{k=1}^{q} \oint_{\Gamma_{k}} \frac{d \zeta S_{p-1}(\zeta)}{F_{p}(\zeta)(\zeta-z)} \\
& =-\frac{F_{p}(z)}{2 \pi i} \sum_{k=1}^{q} \sum_{\ell=0}^{p-1} \frac{S_{p-1}^{(\ell)}\left(\mu_{k}\right)}{\ell !} \oint_{\Gamma_{k}} \frac{d \zeta\left(\zeta-\mu_{k}\right)^{\ell}}{F_{p}(\zeta)(\zeta-z)} \\
& =-\frac{F_{p}(z)}{2 \pi i} \sum_{k=1}^{q} \sum_{\ell=0}^{p-1} \frac{S_{p-1}^{(\ell)}\left(\mu_{k}\right)}{\ell !} \oint_{\Gamma_{k}} \frac{d \zeta\left(\zeta-\mu_{k}\right)^{\ell}}{(\zeta-z) \prod_{k^{\prime}=1}^{q}\left(\zeta-\mu_{k^{\prime}}\right)^{p_{k^{\prime}}}} \\
& =-\frac{F_{p}(z)}{2 \pi i} \sum_{k=1}^{q} \sum_{\ell=0}^{p-1} \frac{S_{p-1}^{(\ell)}\left(\mu_{k}\right)}{\ell !} \oint_{\Gamma_{k}} \frac{d \zeta\left(\zeta-\mu_{k}\right)^{\ell-p_{k}}}{(\zeta-z) \prod_{\substack{k^{\prime}=1 \\
k^{\prime} \neq k}}^{q}\left(\zeta-\mu_{k^{\prime}}\right)^{p_{k^{\prime}}}} \\
& =-\frac{F_{p}(z)}{2 \pi i} \sum_{k=1}^{q} \sum_{\ell=0}^{p_{k}-1} \frac{S_{p-1}^{(\ell)}\left(\mu_{k}\right)}{\ell !} \oint_{\Gamma_{k}} \frac{d \zeta\left(\zeta-\mu_{k}\right)^{\ell-p_{k}}}{(\zeta-z) \prod_{\substack{k^{\prime}=1 \\
k^{\prime} \neq k}}^{q}\left(\zeta-\mu_{k^{\prime}}\right)^{p_{k^{\prime}}}}
\end{aligned}
$$

where we used

$$
\oint_{\Gamma_{k}} d \zeta\left(\zeta-\mu_{k}\right)^{\ell-p_{k}} f(\zeta)=0 \quad \text { for } \ell \geq p_{k}, \ell \in \mathbb{N}
$$

for any function $f$ analytic in a neighborhood of the disk $D_{k}$ with boundary $\Gamma_{k}, k=1, \ldots, q$, to arrive at the last line of (B.9). An application of Cauchy's formula for derivatives of analytic functions to (B.9) then yields

$$
\begin{aligned}
S_{p-1}(z)= & -F_{p}(z) \sum_{k=1}^{q} \sum_{\ell=0}^{p_{k}-1} \frac{S_{p-1}^{(\ell)}\left(\mu_{k}\right)}{\ell !} \\
& \times \frac{1}{2 \pi i} \oint_{\Gamma_{k}} d \zeta \frac{1}{\left(\zeta-\mu_{k}\right)^{\left(p_{k}-\ell-1\right)+1}} \frac{1}{(\zeta-z) \prod_{k^{\prime}=1, k^{\prime} \neq k}^{q}\left(\zeta-\mu_{k^{\prime}}\right)^{p_{k^{\prime}}}} \\
(\mathrm{B} .11)= & F_{p}(z) \sum_{k=1}^{q} \sum_{\ell=0}^{p_{k}-1} \frac{S_{p-1}^{(\ell)}\left(\mu_{k}\right)}{\ell !\left(p_{k}-\ell-1\right) !} \\
& \times\left.\left(\frac{d^{p_{k}-\ell-1}}{d \zeta^{p_{k}-\ell-1}}\left(\frac{1}{(z-\zeta) \prod_{k^{\prime}=1, k^{\prime} \neq k}^{q}\left(\zeta-\mu_{k^{\prime}}\right)^{p_{k^{\prime}}}}\right)\right)\right|_{\zeta=\mu_{k}}, \quad z \in \mathbb{C},
\end{aligned}
$$

and hence (B.2). Conversely, a linear algebraic argument shows that any polynomial $T_{p-1}$ of degree $p-1$ is uniquely determined by data of the type

$$
T_{p-1}\left(\mu_{k}\right), T_{p-1}^{\prime}\left(\mu_{k}\right), \ldots, T_{p-1}^{\left(p_{k}-1\right)}\left(\mu_{k}\right), \quad k=1, \ldots, q .
$$

Uniqueness of the representation (B.2) then proves (B.5). 
We briefly mention two special cases of (B.2). First, assume the generic case where all zeros of $F_{p}$ are distinct, that is,

$$
q=p, \quad p_{k}=1, \quad \mu_{k} \neq \mu_{k^{\prime}} \text { for } k \neq k^{\prime}, k, k^{\prime}=1, \ldots, p .
$$

In this case (B.2) reduces to the classical Lagrange interpolation formula

$$
S_{p-1}(z)=F_{p}(z) \sum_{k=1}^{p} \frac{S_{p-1}\left(\mu_{k}\right)}{\left(\left.\left(d F_{p}(\zeta) / d \zeta\right)\right|_{\zeta=\mu_{k}}\right)\left(z-\mu_{k}\right)}, \quad z \in \mathbb{C} .
$$

Second, we consider the other extreme case where all zeros of $F_{p}$ coincide, that is,

$$
q=1, \quad p_{1}=p, \quad F_{p}(z)=\left(z-\mu_{1}\right)^{p}, \quad z \in \mathbb{C} .
$$

In this case (B.2) reduces of course to the Taylor expansion of $S_{p-1}$ around $z=\mu_{1}$,

$$
S_{p-1}(z)=\sum_{\ell=0}^{p-1} \frac{S_{p-1}^{(\ell)}\left(\mu_{1}\right)}{\ell !}\left(z-\mu_{1}\right)^{\ell}, \quad z \in \mathbb{C}
$$

\section{Asymptotic spectral parameter expansions and non- linear recursion relations}

In this appendix we discuss asymptotic spectral parameter expansions for $F_{p} / y$ and $G_{p+1} / y$ as well as nonlinear recursion relations for the corresponding homogeneous coefficients $\hat{f}_{\ell}$ and $\hat{g}_{\ell}$ and analogous quantities fundamental to the polynomial recursion formalism for the Toda hierarchy.

We start by recalling the following elementary results (which are consequences of the binomial expansion). Let

$$
\begin{aligned}
& \left\{E_{m}\right\}_{m=0, \ldots, 2 p+1} \subset \mathbb{C} \text { for some } p \in \mathbb{N}_{0} \\
& \text { and } \eta \in \mathbb{C} \text { such that }|\eta|<\min \left\{\left|E_{0}\right|^{-1}, \ldots,\left|E_{2 p+1}\right|^{-1}\right\} .
\end{aligned}
$$

Then

$$
\left(\prod_{m=0}^{2 p+1}\left(1-E_{m} \eta\right)\right)^{-1 / 2}=\sum_{k=0}^{\infty} \hat{c}_{k}(\underline{E}) \eta^{k}
$$

where

$$
\begin{aligned}
& \hat{c}_{0}(\underline{E})=1, \\
& \hat{c}_{k}(\underline{E})=\sum_{\substack{j_{0}, \ldots, j_{2 p+1}=0 \\
j_{0}+\cdots+j_{2 p+1}=k}}^{k} \frac{\left(2 j_{0}\right) ! \cdots\left(2 j_{2 p+1}\right) !}{2^{2 k}\left(j_{0} !\right)^{2} \cdots\left(j_{2 p+1} !\right)^{2}} E_{0}^{j_{0}} \cdots E_{2 p+1}^{j_{2 p+1}}, \quad k \in \mathbb{N} .
\end{aligned}
$$


The first few coefficients explicitly read

$$
\begin{aligned}
& \hat{c}_{0}(\underline{E})=1, \\
& \hat{c}_{1}(\underline{E})=\frac{1}{2} \sum_{m=0}^{2 p+1} E_{m}, \\
& \hat{c}_{2}(\underline{E})=\frac{1}{4} \sum_{\substack{m_{1}, m_{2}=0 \\
m_{1}<m_{2}}}^{2 p+1} E_{m_{1}} E_{m_{2}}+\frac{3}{8} \sum_{m=0}^{2 p+1} E_{m}^{2}, \quad \text { etc. }
\end{aligned}
$$

Similarly,

$$
\left(\prod_{m=0}^{2 p+1}\left(1-E_{m} \eta\right)\right)^{1 / 2}=\sum_{k=0}^{\infty} c_{k}(\underline{E}) \eta^{k}
$$

where

$$
\begin{aligned}
c_{0}(\underline{E}) & =1, \\
\text { (C.6) } \quad c_{k}(\underline{E}) & =\sum_{\substack{j_{0}, \ldots, j_{2 p+1}=0 \\
j_{0}+\cdots+j_{2 p+1}=k}}^{k} \frac{\left(2 j_{0}\right) ! \cdots\left(2 j_{2 p+1}\right) ! E_{0}^{j_{0}} \cdots E_{2 p+1}^{j_{2 p+1}}}{2^{2 k}\left(j_{0} !\right)^{2} \cdots\left(j_{2 p+1} !\right)^{2}\left(2 j_{0}-1\right) \cdots\left(2 j_{2 p+1}-1\right)}, \\
& k \in \mathbb{N} .
\end{aligned}
$$

The first few coefficients are given explicitly by

$$
\begin{aligned}
& c_{0}(\underline{E})=1, \\
& c_{1}(\underline{E})=-\frac{1}{2} \sum_{m=0}^{2 p+1} E_{m}, \\
& c_{2}(\underline{E})=\frac{1}{4} \sum_{\substack{m_{1}, m_{2}=0 \\
m_{1}<m_{2}}}^{2 p+1} E_{m_{1}} E_{m_{2}}-\frac{1}{8} \sum_{m=0}^{2 p+1} E_{m}^{2}, \quad \text { etc. }
\end{aligned}
$$

Theorem C.1. Assume (2.1), $\mathrm{s}^{-\mathrm{Tl}_{p}}(a, b)=0$, and suppose $P=(z, y) \in$ $\mathcal{K}_{p} \backslash\left\{P_{\infty_{+}}, P_{\infty_{-}}\right\}$. Then $F_{p} / y$ and $G_{p+1} / y$ have the following convergent expansions as $P \rightarrow P_{\infty_{ \pm}}$,

$$
\frac{F_{p}(z)}{y}=\mp \sum_{\ell=0}^{\infty} \hat{f}_{\ell} \zeta^{\ell+1}, \quad \frac{G_{p+1}(z)}{y}=\mp \sum_{\ell=-1}^{\infty} \hat{g}_{\ell} \zeta^{\ell+1},
$$


where $\zeta=1 / z$ is the local coordinate near $P_{\infty_{ \pm}}$and $\hat{f}_{\ell}$ and $\hat{g}_{\ell}$ are the homogeneous versions of the coefficients $f_{\ell}$ and $g_{\ell}$ introduced in (2.8). In particular, $\hat{f}_{\ell}$ and $\hat{g}_{\ell}$ can be computed from the nonlinear recursion relations

$$
\begin{aligned}
\hat{f}_{0}=1 & \\
\hat{f}_{1}=- & b \\
\hat{f}_{2}=a^{2} & +\left(a^{-}\right)^{2}+b^{2} \\
(\mathrm{C} .9) \quad \hat{f}_{\ell+2}= & -\frac{1}{2} \sum_{k=1}^{\ell+1} \hat{f}_{\ell+2-k} \hat{f}_{k}-2 b \sum_{k=0}^{\ell+1} \hat{f}_{\ell+1-k} \hat{f}_{k} \\
& +\sum_{k=0}^{\ell}\left(-3 b^{2} \hat{f}_{\ell-k} \hat{f}_{k}+a^{2} \hat{f}_{\ell-k}^{+} \hat{f}_{k}+\left(a^{-}\right)^{2} \hat{f}_{\ell-k} \hat{f}_{k}^{-}\right) \\
& +\sum_{k=0}^{\ell-1}\left(-2 b^{3} \hat{f}_{\ell-1-k} \hat{f}_{k}+2 a^{2} b \hat{f}_{\ell-1-k}^{+} \hat{f}_{k}+2\left(a^{-}\right)^{2} b \hat{f}_{\ell-1-k} \hat{f}_{k}^{-}\right) \\
& +\sum_{k=0}^{\ell-2}\left(a^{2} b^{2} \hat{f}_{\ell-2-k}^{+} \hat{f}_{k}+\left(a^{-}\right)^{2} b^{2} \hat{f}_{\ell-2-k} \hat{f}_{k}^{-}+a^{2}\left(a^{-}\right)^{2} \hat{f}_{\ell-2-k}^{+} \hat{f}_{k}^{-}\right. \\
& \left.-\frac{1}{2} a^{4} \hat{f}_{\ell-2-k}^{+} \hat{f}_{k}^{+}-\frac{1}{2}\left(a^{-}\right)^{4} \hat{f}_{\ell-2-k}^{-} \hat{f}_{k}^{-}\right), \quad \ell \in \mathbb{N},
\end{aligned}
$$

and

$$
\begin{aligned}
\hat{g}_{-1} & =-1 \\
\hat{g}_{0}= & 0 \\
\hat{g}_{1}= & -2 a^{2} \\
(\mathrm{C} .10) \quad \hat{g}_{\ell+1} & =\frac{1}{2} \sum_{k=-1}^{\ell}\left(b+b^{+}\right) \hat{g}_{\ell-1-k} \hat{g}_{k}+\frac{1}{2} \sum_{k=0}^{\ell} \hat{g}_{\ell-k} \hat{g}_{k} \\
& +\frac{1}{2} \sum_{k=-1}^{\ell-1}\left(b b^{+} \hat{g}_{\ell-2-k} \hat{g}_{k}-a^{2}\left(\hat{g}_{\ell-2-k}^{-}+\hat{g}_{\ell-2-k}\right)\left(\hat{g}_{k}+\hat{g}_{k}^{+}\right)\right), \quad \ell \in \mathbb{N} .
\end{aligned}
$$

Moreover, one infers for the $E_{m}$-dependent summation constants $c_{\ell}, \ell=$ $0, \ldots, p+1$, in $F_{p}$ and $G_{p+1}$ that

$$
c_{\ell}=c_{\ell}(\underline{E}), \quad \ell=0, \ldots, p+1
$$


$a n d^{3}$

$$
\begin{aligned}
f_{\ell}= & \sum_{k=0}^{\ell} c_{\ell-k}(\underline{E}) \hat{f}_{k}, \quad \ell=0, \ldots, p, \\
g_{\ell}+f_{p+1} \delta_{p, \ell}= & \sum_{k=0}^{\ell} c_{\ell-k}(\underline{E}) \hat{g}_{k}-c_{\ell+1}(\underline{E}), \quad \ell=0, \ldots, p, \\
\hat{f}_{\ell}= & \sum_{k=0}^{\ell \wedge p} \hat{c}_{\ell-k}(\underline{E}) f_{k}, \quad \ell \in \mathbb{N}_{0}, \\
\hat{g}_{\ell}= & \sum_{k=0}^{\ell \wedge p} \hat{c}_{\ell-k}(\underline{E})\left(g_{k}+f_{p+1} \delta_{p, k}\right)-\hat{c}_{\ell+1}(\underline{E}), \quad \ell \in \mathbb{N}_{0} .
\end{aligned}
$$

Proof. Dividing $F_{p}$ and $G_{p+1}$ by $R_{2 p+2}^{1 / 2}$ (temporarily fixing the branch of $R_{2 p+2}^{1 / 2}$ as $z^{p+1}$ near infinity) one obtains

$$
\begin{aligned}
& \frac{F_{p}(z)}{R_{2 p+2}(z)^{1 / 2}} \underset{|z| \rightarrow \infty}{=}\left(\sum_{k=0}^{\infty} \hat{c}_{k}(\underline{E}) z^{-k}\right)\left(\sum_{\ell=0}^{p} f_{\ell} z^{-\ell-1}\right)=\sum_{\ell=0}^{\infty} \check{f}_{\ell} z^{-\ell-1} \\
& \frac{G_{p+1}(z)}{R_{2 p+2}(z)^{1 / 2}} \underset{\substack{|z| \rightarrow \infty \\
=}}{=}\left(\sum_{k=0}^{\infty} \hat{c}_{k}(\underline{E}) z^{-k}\right)\left(\sum_{\ell=0}^{p+1} \tilde{g}_{\ell} z^{-\ell}\right)=z^{-1} \sum_{\ell=-1}^{\infty} \check{g}_{\ell} z^{-\ell}
\end{aligned}
$$

for some coefficients $\check{f}_{\ell}$ and $\check{g}_{\ell}$ to be determined next. Here we have temporarily introduced the notation

$$
G_{p+1}(z)=-z^{p+1}+\sum_{\ell=0}^{p} g_{p-\ell} z^{\ell}+f_{p+1}=\sum_{\ell=0}^{p+1} \tilde{g}_{p-\ell} z^{\ell} .
$$

Dividing (2.37) and (2.39) by $R_{2 p+2}$ and inserting expansions (C.16) and (C.17) into the resulting equations then yield the nonlinear recursion relations (C.9) and (C.10) (with $\hat{f}_{\ell}$ and $\hat{g}_{\ell}$ replaced by $\check{f}_{\ell}$ and $\check{g}_{\ell}$, respectively). More precisely, one first obtains $\left|\check{f}_{0}\right|=\left|\check{g}_{-1}\right|=1$ and upon choosing the signs of $\check{f}_{0}$ and $\check{g}_{-1}$ such that $\check{f}_{0}=\hat{f}_{0}=1$ and $\check{g}_{-1}=-1$ one obtains (C.9) and (C.10). Next, dividing (2.31) and (2.32) by $R_{2 p+2}^{1 / 2}$, inserting the expansions (C.16) and (C.17), and comparing powers of $z^{-\ell}$ as $z \rightarrow \infty$, one infers that $\check{f}_{\ell}$ and $\check{g}_{\ell}$ satisfy the linear recursion relations (2.4)-(2.6). Hence one concludes that

$$
\check{f}_{\ell}=f_{\ell}, \quad \check{g}_{\ell}=g_{\ell}, \quad \ell \in \mathbb{N}_{0}
$$

\footnotetext{
${ }^{3} m \wedge n=\min \{m, n\}$.
} 
for certain values of the summation constants $c_{\ell}$. To show that $\check{f}_{\ell}=\hat{f}_{\ell}$, $\check{g}_{\ell}=\hat{g}_{\ell}$, and hence all $c_{\ell}, \ell \in \mathbb{N}$, vanish, we recall the notion of degree as used in the proof of Lemma 5.4, which serves as an efficient tool to distinguish between homogeneous and nonhomogeneous quantities. To this end we employ the notation

$$
f^{(r)}=S^{(r)} f, \quad f=\{f(n)\}_{n \in \mathbb{Z}} \subset \mathbb{C}, \quad S^{(r)}=\left\{\begin{array}{ll}
\left(S^{+}\right)^{r}, & r \geq 0, \\
\left(S^{-}\right)^{-r}, & r<0,
\end{array} \quad r \in \mathbb{Z},\right.
$$

and introduce

$$
\operatorname{deg}\left(a^{(r)}\right)=\operatorname{deg}\left(b^{(r)}\right)=1, \quad r \in \mathbb{Z} .
$$

This results in

$$
\operatorname{deg}\left(\hat{f}_{\ell}\right)=\ell, \quad \operatorname{deg}\left(\hat{g}_{\ell}\right)=\ell+1, \quad \ell \in \mathbb{N} .
$$

using induction in the linear recursion relations (2.4)-(2.6). Similarly, the nonlinear recursion relations (C.9) and (C.10) yield inductively that

$$
\operatorname{deg}\left(\check{f}_{\ell}\right)=\ell, \quad \operatorname{deg}\left(\check{g}_{\ell}\right)=\ell+1, \quad \ell \in \mathbb{N} .
$$

Hence one concludes that

$$
\check{f}_{\ell}=\hat{f}_{\ell}, \quad \check{g}_{\ell}=\hat{g}_{\ell}, \quad \ell \in \mathbb{N}_{0} .
$$

A comparison of coefficients in (C.16) proves (C.14). Similarly, we use (C.17) to establish (C.15). Next, multiplying (C.2) and (C.5), a comparison of coefficients of $\eta^{k}$ yields

$$
\sum_{\ell=0}^{k} \hat{c}_{k-\ell}(\underline{E}) c_{\ell}(\underline{E})=\delta_{k, 0}, \quad k \in \mathbb{N}_{0} .
$$

Thus, one computes

$$
\begin{aligned}
\sum_{m=0}^{\ell} c_{\ell-m}(\underline{E}) \hat{f}_{m} & =\sum_{m=0}^{\ell} \sum_{k=0}^{m} c_{\ell-m}(\underline{E}) \hat{c}_{m-k}(\underline{E}) f_{k} \\
& =\sum_{k=0}^{\ell} \sum_{p=k}^{\ell} c_{\ell-p}(\underline{E}) \hat{c}_{p-k}(\underline{E}) f_{k} \\
& =\sum_{k=0}^{\ell}\left(\sum_{m=0}^{\ell-k} c_{\ell-k-m}(\underline{E}) \hat{c}_{m}(\underline{E})\right) f_{k}=f_{\ell}, \quad \ell=0, \ldots, p
\end{aligned}
$$

applying (C.25). Hence one obtains (C.12) and thus (C.11) (cf. (2.9)). The corresponding proof of (C.13) is similar to that of $f_{\ell}$. 
Acknowledgments. We are indebted to Michael Gekhtman for discussions on this subject. Fritz Gesztesy gratefully acknowledges the extraordinary hospitality of Helge Holden and the Department of Mathematical Sciences of the Norwegian University of Science and Technology, Trondheim, during a two-month stay in the summer of 2005, where parts of this paper were written. He also gratefully acknowledges a research leave for the academic year 2005/06 granted by the Research Council and the Office of Research of the University of Missouri-Columbia.Fritz Gesztesy and Helge Holden are grateful for the hospitality of the Mittag-Leffler Institute, Sweden, creating a great working environment for research, during the Fall of 2005. Gerald Teschl gratefully acknowledges the hospitality of the Department of Mathematics of the University of Missouri-Columbia and the Department of Mathematical Sciences of the Norwegian University of Science and Technology, Trondheim, respectively, during two one-week stays in 2005.

\section{References}

[1] Abraham, R., Marsden, J.E. and Ratiu, T.: Manifolds, Tensor Analysis, and Applications, 2nd ed. Applied Mathematics Sciences 75. Springer-Verlag, New York, 1988.

[2] Arnold, V. I.: Mathematical Methods of Classical Mechanics, 2nd ed. Graduate Text in Mathematics 60. Springer-Verlag, New York, 1989.

[3] Batchenko, V. and Gesztesy, F.: On the spectrum of Schrödinger operators with quasi-periodic algebro-geometric KdV potentials. J. Anal. Math. 95 (2005), 333-387.

[4] Batchenko, V. And Gesztesy, F.: On the spectrum of Jacobi operators with quasi-periodic algebro-geometric coefficients. IMRP Int. Math. Res. Pap. (2005), no. 10, 511-563.

[5] Belokolos, E. D., Bobenko, A. I., Enol'skit, V.Z., Its, A. R. And Matveev, V. B.: Algebro-Geometric Approach to Nonlinear Integrable Equations. Springer, Berlin, 1994.

[6] Bulla, W., Gesztesy, F., Holden, H. and Teschl, G.: Algebrogeometric quasi-periodic finite-gap solutions of the Toda and Kac-van Moerbeke hierarchies. Mem. Amer. Math. Soc. 135 (1998), no. 641, 1-79.

[7] Date, E. And Tanaka, S.: Analogue of inverse scattering theory for the discrete Hill's equation and exact solutions for the periodic Toda lattice. Progr. Theoret. Phys. 55 (1976), no.2, 457-465.

[8] Dubrovin, B. A.: Theta functions and non-linear equations. Russian Math. Surveys 36:2 (1981), 11-92.

[9] Dubrovin, B. A., Krichever, I. M. and Novikov, S. P.: Integrable systems I. In Dynamical Systems IV, 173-332 (V.I. Arnold and S.P. Novikov, eds.). Springer, Berlin, 1990. 
[10] Dubrovin, B. A., Matveev, V. B. And Novikov, S. P.: Non-linear equations of the Korteweg-de Vries type, finite-zone linear operators and Abelian varieties. Russ. Math. Surveys 31 (1976), no. 1, 59-146.

[11] Farkas, H. And Kra, I.: Riemann Surfaces. Graduate Text in Mathematics 71. Springer-Verlag, New York, 1992.

[12] Flaschka, H.: Discrete and periodic illustrations of some aspects of the inverse method. In Dynamical Systems, Theory and Applications (Rencontres, Batelle Res. Inst., Seattle, Wash., 1974), 441-466. Lecture Notes in Physics 38. Springer, Berlin, 1975.

[13] Gesztesy, F. And Holden, H.: Soliton Equations and Their AlgebroGeometric Solutions. Vol. I: (1+1)-Dimensional Continuous Models. Cambridge Studies in Advanced Mathematics 79. Cambridge University Press, Cambridge, 2003.

[14] Gesztesy, F., Holden, H., Michor, J. And Teschl, G.: Soliton Equations and Their Algebro-Geometric Solutions. Vol. II: $(1+1)$-Dimensional Discrete Models. Cambridge Studies in Advanced Mathematics. Cambridge Univ. Press, in press.

[15] Kac, M. and Van Moerbeke, P.: On some periodic Toda lattices. Proc. Nat. Acad. Sci. USA 72 (1975), 1627-1629.

[16] Kac, M. And Van Moerbeke, P.: A complete solution of the periodic Toda problem. Proc. Nat. Acad. Sci. USA 72 (1975), no. 8, 2879-2880.

[17] Katok, A. and Hasselblatt, B.: Introduction to the Modern Theory of Dynamical Systems. Encyclopedia of Mathematics and its Applications 54. Cambridge University Press, Cambridge, 1995.

[18] Krichever, I. M.: Algebraic curves and non-linear difference equations. Russ. Math. Surveys 33 (1978), no.4, 255-256.

[19] KričEver, I. M.: Algebro-geometric spectral theory of the Schrödinger difference operator and the Peierls model. Sov. Math. Dokl. 26 (1982), 194-198.

[20] Krichever, I. M.: The Peierls model. Funct. Anal. Appl. 16 (1982), 248-263.

[21] Krichever, I. M.: Nonlinear equations and elliptic curves. Revs. Sci. Tech. 23 (1983), 51-90.

[22] Markushevich, A. I.: Theory of Functions of a Complex Variable, 2nd. ed. Chelsea Publishing Co., New York, 1985.

[23] McKean, H. P.: Integrable systems and algebraic curves. In Global Analysis (Proc. Biennial Sem. Canad. Math. Congr., Univ. Calgary, Calgary, Alta., 1978), 83-200. M. Grmela and J. E. Marsden (eds.). Lecture Notes in Math. 755. Springer, Berlin, 1979.

[24] Van Moerbeke, P.: The spectrum of Jacobi matrices. Invent. Math. 37 (1976), no. $1,45-81$. 
[25] Van Moerbeke, P. And Mumford, D.: The spectrum of difference operators and algebraic curves. Acta Math. 143 (1979), no. 1-2, 93-154.

[26] Mumford, D.: An algebro-geometric construction of commuting operators and of solutions to the Toda lattice equation, Korteweg deVries equation and related non-linear equations In Proceedings of the International Symposium on Algebraic Geometry (Kyoto Univ., Kyoto, 1977) 115-153. Kinokuniya Book Store, Tokyo, 1978.

[27] Mumford, D.: Tata Lectures on Theta II. Progress in Mathematics 43. Birkhäuser Boston, Boston, MA, 1984.

[28] Nắman, P. B.: On the theory of periodic and limit-periodic Jacobian matrices. Sov. Math. Dokl. 3 (1962), 383-385.

[29] NAĬMAN, P. B.: On the spectral theory of non-symmetric periodic Jacobi matrices. Zap. Meh.-Mat. Fak. Harprime kov. Gos. Univ. i Harprime kov. Mat. Ob̌̌č. (4) 30 (1964), 138-151. (Russian.)

[30] Novikov, S., Manakov, S. V., Pitaevskit, L. P. and Zakharov, V. E.: Theory of Solitons. Contemporary Soviet Mathematics. Consultants Bureau, New York, 1984.

[31] Pastur, L. And Figotin, A.: Spectra of Random and AlmostPeriodic Operators. Grundlehren der Mathematischen Wissenschaften 297. Springer-Verlag, Berlin, 1992.

[32] Teschl, G.: Jacobi Operators and Completely Integrable Nonlinear Lattices. Mathematical Surveys and Monographs 72. American Mathematical Society, Providence, R.I., 2000.

[33] TodA, M.: Theory of Nonlinear Lattices. Springer Series in Solid-State Sciences 20. Springer-Verlag, Berlin, 1989.

[34] Toda, M.: Nonlinear Waves and Solitons. Mathematics and its Applications (Japanese Series) 5. Kluwer Academic Publishers Group, Dordrecht, 1989.

[35] Walter, W.: Ordinary Differential Equations. Graduate Texts in Mathematics 182. Springer-Verlag, New York, 1998.

Recibido: 28 de marzo de 2006

Fritz Gesztesy

Department of Mathematics

University of Missouri

Columbia, MO 65211, USA

fritz@math.missouri.edu

http://www .math.missouri.edu/personnel/faculty/gesztesyf.html 
182 F. Gesztesy, H. Holden and G. Teschl

Helge Holden

Department of Mathematical Sciences Norwegian University of Science and Technology NO-7491 Trondheim, Norway holden@math.ntnu.no http://www.math.ntnu.no/ 〜holden/

Gerald Teschl Faculty of Mathematics

University of Vienna Nordbergstrasse 15, 1090 Wien, Austria

and

International Erwin Schrödinger Institute for Mathematical Physics

Boltzmanngasse 9, 1090 Wien, Austria

Gerald.Teschl@univie.ac.at http://www.mat.univie.ac.at/〜gerald/

Research supported in part by the Research Council of Norway, the US National Science Foundation under Grant No. DMS-0405526, and the Austrian Science Fund (FWF) under Grant No. P17762. 